\title{
NEW GENERA AND SPECIES, AND NEW RECORDS, OF TASMANIAN TRICHOPTERA (INSECTA)
}

\author{
by Arturs Neboiss
}

(with 28 text-figures)

\begin{abstract}
Neboiss, A., 2002 (31:xii): New genera and species, and new records of Tasmanian Trichoptera (Insecta). Papers and Proceedings of the Royal Society of Tasmania 136: 43-82. https://doi.org/10.26749/rstpp.136.43

ISSN 0080 4703. Department of Entomology, Museum Victoria, Melbourne 3001, Australia.
\end{abstract}

\begin{abstract}
Examination of Trichoptera specimens collected in Tasmania over the last 25 years has led to a revision of the Trichoptera list for Tasmania, and expansion of the distributions of many species, particularly those in the World Heritage areas. The following 17 new species are described: Poecilochorema anastemum, Taschorema dispatens, Ptychobiosis iconica, Chimarra derogata, Hydrobiosella tahunense, H. anatolica, H. disrupta, H. propinqua, H. scalaris, H. otaria, Daternomina quastrulla, D. jacksonae, Cheumatopsyche deani, C. alampeta, Diemeniluma serrula, Marilia aenigmata and Tasmanthrus gwendolensis. There are also three new combinations - Taschorema ithyphallicum Schmid, Daternomina irrorata Kimmins and Diemeniluma tasmanica Jacquemart. New genera are established for Daternomina (Family Ecnomidae) and Diemeniluma (Family Hydropsychidae).
\end{abstract}

Key Words: Trichoptera, Tasmanian World Heritage areas, classification, new genera and species.

\section{INTRODUCTION}

The present study of Tasmanian caddisflies (Trichoptera) introduces new genera and species and extends distributions of previously recorded species. It results from evaluation of material accumulated during the last 25 years, during which time, considerable effort has been spent investigating the Tasmanian World Heritage areas (Dean \& Cartwright 1992, Jackson 1999, 2000). Over this period, too, there have been dramatic changes to the environment, particularly to the water systems associated with the Gordon River dam, and, significantly, some of the species included in this study are currently listed in the Threatened Species Protection Act 1995 (Tas). In addition, further development and analyses of Trichoptera systematics (Neboiss 1986, Schmid 1989) have led to taxonomic changes that impinge on the Tasmanian Trichoptera faunal list, necessitating a reassessment of the generic and specific status of several species. With the description here of two new genera, Daternomina (FamilyEcnomidae) and Diemeniluma (Family Hydropsychidae), and 17 new species, the Tasmanian fauna now totals 189 species in 74 genera. New information is given on species' distributions.

\section{MATERIAL AND METHODS}

All specimens are preserved in $75 \%$ ethanol; if detailed examination was required, abdomens were cleared in $\mathrm{KOH}$ solution and then transferred to glycerol for drawing and storage. Dissected specimens are identified by the author's notebook number with the prefix 'PT-'. All examined material is in the Museum Victoria collection, Melbourne (NMV), unless otherwise indicated. Other depository institutions are identified as follows: ANIC - Australian National Insect Collection, Canberra; BMNH - The Natural History Museum, London (= NMH); IRScNB Institut Royal des Sciences Naturelles de Belgique, Brussels; QM - Queensland Museum, Brisbane; and SAM — South Australian Museum, Adelaide.

\section{FAMILY HYDROBIOSIDAE}

The family Hydrobiosidae was revised by Schmid (1989), who regarded it as a primitive family of Gondwanan origin. Schmid, however, admitted that the phylogeny could not be reconstructed, and placed hydrobiosids, with uncertainty, at several positions in the Annulipalpia branch (Schmid 1989: fig. 33). In the same work, Schmid described several new species from Tasmaniaand split the genus Austrochorema Mosely into two separate genera: Austrochorema s.l., and Poecilochorema Schmid, a genus endemic to Tasmania. Further changes proposed here include the referral of Tasmanian specimens previously identified as Ptychobiosis nigrita Banks to a new species - iconica; transfer of Ethochoremaithyphallicum Schmid to thegenus Taschorema; and description of another new species, dispatens.

\section{Genus Allochorema Mosely}

Allochorema Mosely in Mosely \& Kimmins, 1953: 491; Neboiss 1977: 13.

Type species: Allochorema tasmanica Mosely, by original designation.

\section{Allochorema tasmanicum Mosely}

Allochorema tasmanica Mosely in Mosely \& Kimmins, 1953: 491; Neboiss 1977: 13.

\section{Holotype}

o Tasmania, Mt Wellington, alt. 3000 ft, Dec. 1937 $(\mathrm{BMNH})$

\section{Additional material examined}

$0^{\circ}$ Hartz Mountains National Park, 1977, J, Sedlacek; $40^{\circ}$ Strathgordon, Jan. 1977, J. Sedlacek; $10^{\circ}$ d'Entrecasteaux River source, below Mt LaPerouse, alt. $840 \mathrm{~m}$; 4330'S $146^{\circ} 44^{\prime} E$, 15 Feb. 1988, P.B. McQuillan; $30^{\circ}$ Lake Tahune, Frenchmans Cap area, 28 Jan. 1988, J. Jackson; $10^{\top}$ Lake Pedder, shore N of Forest Creek, 10 Feb. 1999, J. Jackson; 


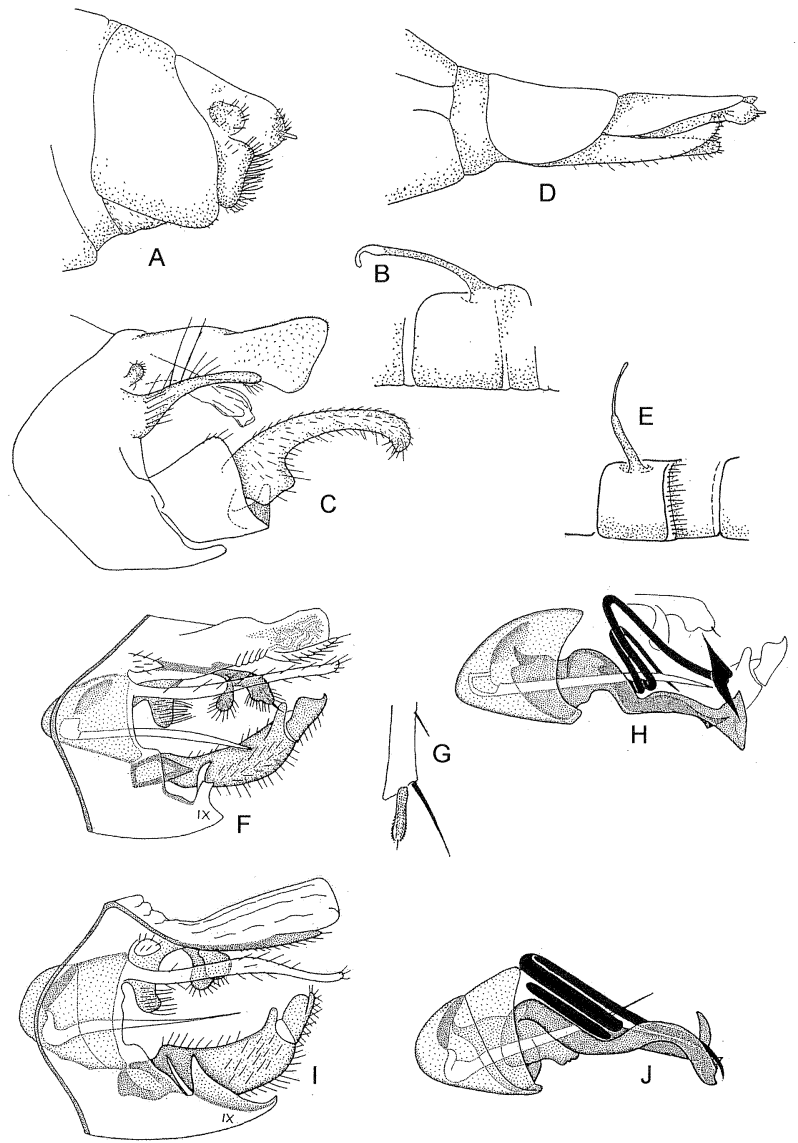

FIG. $1-(A-C)$ Austrochorema pegidion: (A) female genitalia lateral; (B) male lateral filament of sternite $V$; (C) male genitalia lateral (phallic apparatus omitted); (D) Poecilochorema crinitum, female genitalia lateral.; (E) Poecilochorema evansi, male lateral filament of sternite $V$; $(F-H)$ Poecilochorema complexum: $(F)$ male genitalia lateral (phallic apparatus omitted); $(G)$ phallic apparatus lateral; (H) modified spur on male hind tibia; (I, J) Poecilochorema circumvolutum: (I) male genitalia lateral; (J) phallic apparatus lateral.

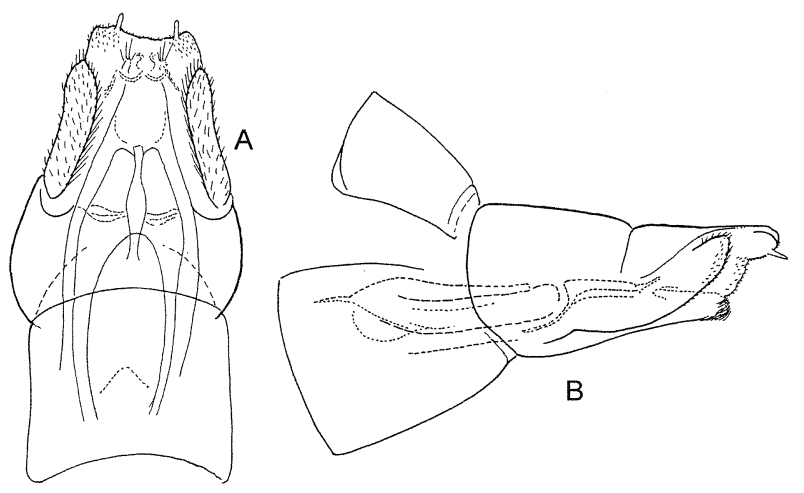

FIG. 3 - Poecilochorema anastemum female genitalia: (A) ventral, (B) lateral.

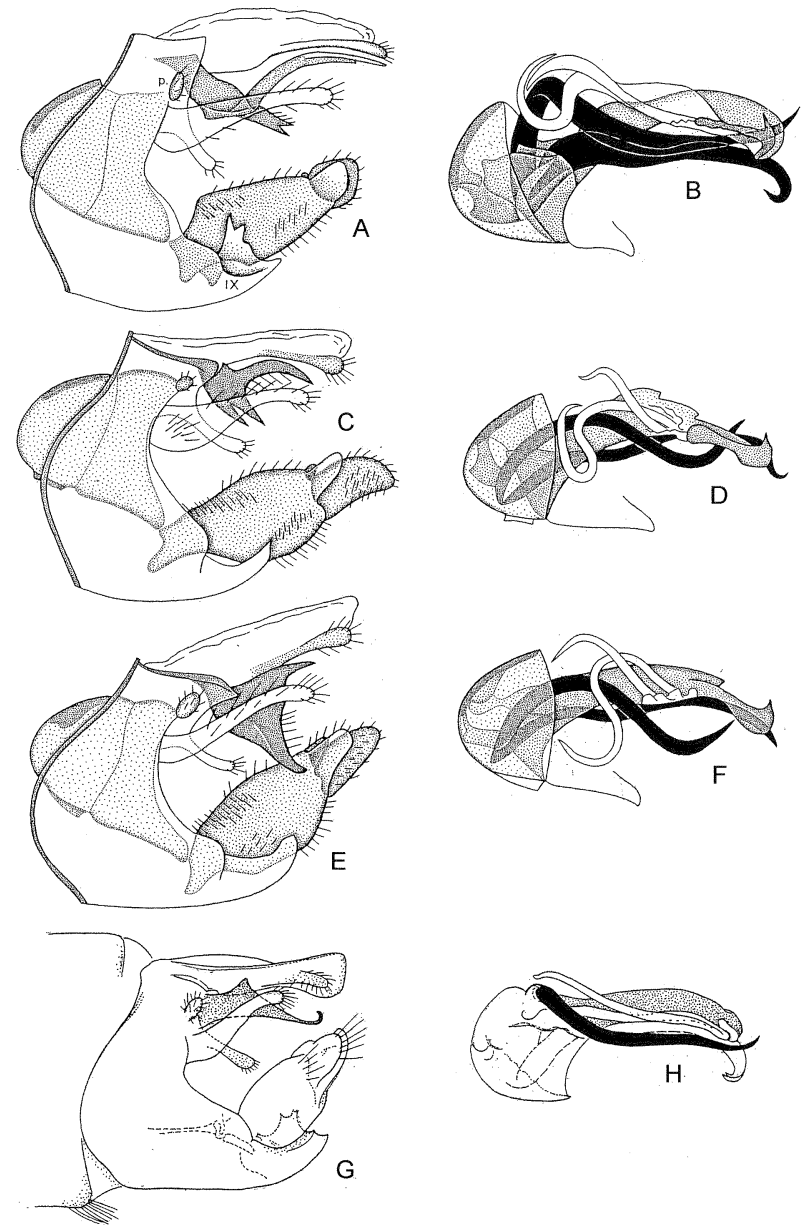

FIG. 2 - (A, B) Poecilochorema evansi: (A) male genitalia lateral; (B) phallic apparatus lateral; $(C, D)$ Poecilochorema crinitum: (C) male genitalia lateral; (D) phallic apparatus lateral; $(E, F)$ Poecilochorema lepnevae: $(E)$ male genitalia lateral; $(F)$ phallic apparatus lateral; $(G, H)$ Poecilochorema anastemum sp. n.: (G) male genitalia lateral; $(H)$ phallic apparatus lateral.

$10^{\star}$ Lake Pedder, Frankland shore, 10 Feb. 1999, J. Jackson; $10^{\pi}$ Lake Oberon, Western Arthur Ranges, top of plain, 9 Mar. 1999, J. Jackson. All specimens in NMV.

These additional locality records indicate that this species has a wider distribution throughout the southwest of Tasmania than believed previously.

Distribution

Tasmania. Not recorded from other states.

\section{Genus Austrochorema Mosely}

Austrochorema Mosely in Mosely \& Kimmins, 1953: 481; Neboiss 1977: 14; Schmid 1989: 76.

Type species

Austrochorema wenta Mosely, by original designation.

Two species groups were recognised by Neboiss (1977), distinguished by differences in male and female genitalic 
structures. In one group, the male genitalia have the inferior appendages with coxopodite short and the terminal segments of the female abdomen short (fig. 1A). The main distribution of this group is on the Australian mainland, with the exception of one species - Austrochorema pegidion Neboiss, which is the only species present in Tasmania. The six other species, all having the coxopodite long in male genitalia and terminal segments elongate and dorsoventrally compressed in the female, constitute the second group. Schmid (1989) analysed these two groups in detail, considering them to be two distinct genera. Poecilochorema included five endemic Tasmanian species. Here another new species is added to this genus.

\section{Austrochorema pegidion Neboiss Figs $1 \mathrm{~A}-\mathrm{C}, 4 \mathrm{~A}$}

Austrochorema pegidion Neboiss, 1962: 535; Neboiss 1977: 15.

\section{Holotype}

OT Tasmania, Broad River nr Lake Dobson, Mt Field National Park, 6 Nov. 1955 (NMV).

\section{Additional localities}

$10^{\top}$ Lake Tahune, Frenchmans Cap area, 29 Jan. 1988, J. Jackson; $10^{*}$ Lake Nancy, Frenchmans Cap area, 28 Jan. 1988, J. Jackson; $10^{\star}$ unnamed creek, Overland track approx. $1.5 \mathrm{~km}$ NW of Pelion Gap, Cradle Mountain-Lake St Clair National Park, 17. Jan. 1990, J. Dean and D. Cartwright; $10^{\star} \mathrm{Mt}$ Barrow, $11 \mathrm{~km}$ EbyN Nunamara, 30 Jan. 1983, J. Cardale (male genitalia prep. PT-2132 illustrated). All specimens in NMV.

Larvae, identified by J. Dean, have been recorded from the following localities: Mt Wellington below Organ Pipes, 21 Sept. 1978; N of Savage River on pipeline, 11 May 1975; Dip River Falls, 27 Feb. 1982; St Columba Falls, 10 Mar. 1982; Rafferty Creek, Ben Lomond National Park, 25 Jan. 1990; Port Davey Track, 2 miles W of Picton River, 11 Feb. 1966; Flinders Island, Mt Strzelecki, Creek off main road, 19 Oct. 1996. All specimens in NMV.

Distribution

Tasmania. Tasmanian endemic.

\section{Genus Poecilochorema Schmid}

Poecilochorema Schmid, 1989: 77.

\section{Type species}

Anachorema evansi Mosely, 1953 by original designation.

This genus was described in great detail by Schmid (1989), and thus only a short diagnosis is given here.

\section{Diagnosis}

Forewing discoidal cell short, open; fork 4 with long footstalk; hind wing fork 1 with long footstalk, fork 2 with short footstalk. In the male, abdominal sternite $\mathrm{V}$ the lateral filament (fig. 1E) has only basal half sclerotised, distal part filiform; inferior appendages with coxopodite longer than harpago; phallic apparatus (the most complex in the family) with long, twisted processes (fig. $1 \mathrm{G}$ ). Female abdomen (fig. 1D) with terminal segments extended distally, dorsoventrally compressed.

The five species included in the genus Poecilochorema by Schmid (1989) were placed in two species groups. The complexum group, including complexum (Jacquemart) and circumvolutum Schmid, is characterised by males with modified posterior mesal spur on hind tibia (fig. $1 \mathrm{H}$ ) and parapodes in male genitalia developed into a pair of distally rounded lobes. In the evansi group, which includes evansi (Mosely), crinitum (Neboiss), lepnaevae (Jacquemart) and anastemum sp. n., the apical mesal spurs are not modified and the parapodes are pointed distally.

The known distributions of the species indicate that the genus consists of several closely related species, each with a rather restricted distribution. Illustrations of males of all species are provided to facilitate their identification. The illustrations are from Schmid (1989) and Neboiss (1977).

\section{complexum group}

\section{Poecilochorema complexum (Jacquemart) Figs $1 \mathrm{~F}-\mathrm{H}, 4 \mathrm{~B}$}

Austrochorema complexa Jacquemart, 1965: 41; Neboiss 1977: 17.

Poecilochorema complexum. Schmid 1989: 134.

\section{Holotype}

OT Tasmania, Cradle Mountain, 10 Jan. 1923 (IRScNB).

\section{Additional localities}

10 Lake Pedder (before flooding), 10 Mar. 1972, A. Neboiss; $10^{7}$ Gordon River $1 \mathrm{~km}$ above First Split, 11 Jan. 1977, A. Neboiss; $10^{\top}$ Franklin River, Roaring Creek junction, $1 \mathrm{~km}$ above Gordon River, 8 Jan. 1977, A. Neboiss.

\section{Distribution}

Tasmania, NW and SW provinces, known from a few localities, rare. Tasmanian endemic.

\section{Poecilochorema circumvolutum Schmid Figs $1 \mathrm{I}, 1 \mathrm{~J}, 4 \mathrm{~B}$}

Poecilochorema circumvolutum Schmid, 1989: 134.

Holotype

ơ Tasmania, Sir John Falls, Cataract Creek, 9 Jan. 1977 (NMV).

\section{Distribution}

Tasmania, SW province, rare, known only from type locality. Tasmanian endemic.

\section{evansi group}

\section{Poecilochorema evansi (Mosely) Figs 1E, 2A, 2B, 4C}

Anachorema evansi Mosely in Mosely \& Kimmins, 1953: 460.

Austrochorema evansi. Neboiss 1962: 533; Neboiss 1977: 15. 

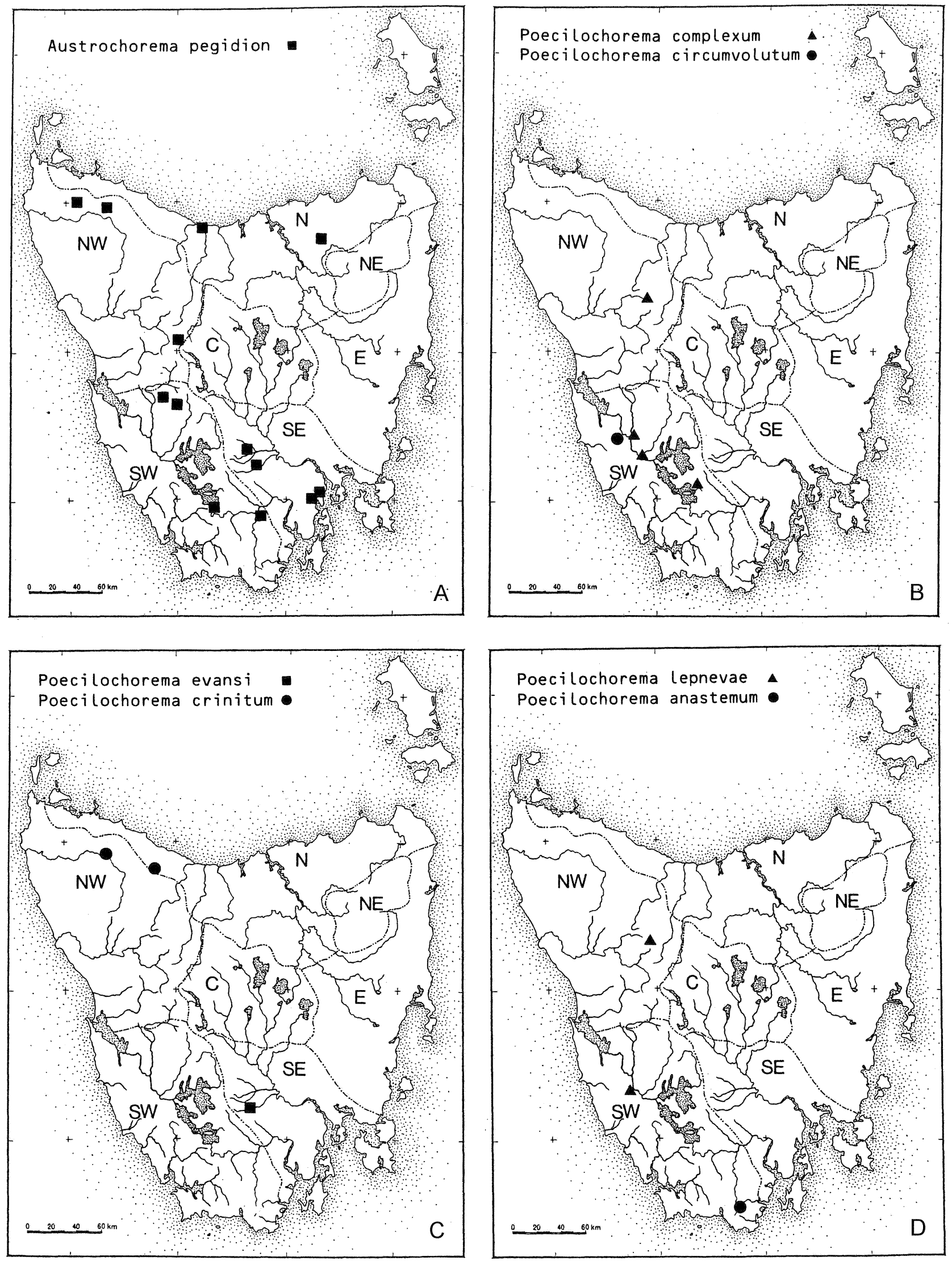

FIG. 4 - Distribution maps: (A) Austrochorema pegidion; (B) Poecilochorema complexum and P. circumvolutum; (C) P. evansi and P. crinitum; (D) P. lepvevae and P. anastemum sp. $n$. 
Poecilochorema evansi. Schmid 1989: 134.

Holotype

OT Tasmania, without definite locality or date (BMNH).

No additional material available.

Distribution

Tasmania, recorded only from Russell Falls, 23 Feb. 1967;

20 Feb. 1971; 5 Dec. 1972. Tasmanian endemic.

\section{Poecilochorema crinitum (Neboiss) Figs 1D, 2C, 2D, 4C}

Austrochorema crinitum Neboiss, 1977: 16.

Poecilochorema crinitum. Schmid 1989: 134.

Holotype

ơ Tasmania, Guide River Falls nr Ridgley, 18 Nov. 1972 (NMV).

\section{Distribution}

Tasmania, Dip River Falls near Mawbanna. No additional material available. Tasmanian endemic.

\section{Poecilochorema lepnevae (Jacquemart) Figs 2E, 2F, 4D}

Austrochorema lepnevae Jacquemart 1965: 43; Neboiss 1977: 17.

Poecilochorema lepnevae. Schmid 1989: 134.

Holotype

O Tasmania, Cradle Mountain, 27 Jan. 1923 (IRScNB).

Distribution

Tasmania, 150 Sir John Falls, Cataract Creek, 8 Jan. 1977, A. Neboiss et al. (may be locally common). No additional specimens available. Tasmanian endemic.

\section{Poecilochorema anastemum sp. $\mathrm{n}$. Figs 2G-3B, 4D}

\section{Type material}

Holotype $0^{\prime}$ Tasmania, below Maxwell Ridge, small tributary to Picton River, above Reservoir Lakes, $43^{\circ} 29^{\prime} \mathrm{S} 146^{\circ} 44^{\prime} \mathrm{E}$, alt. 800 m (Mt LaPerouse area), 15 Feb. 1988, A. Neboiss. Paratypes $20^{*} 40$ collected with holotype (male genitalia prep. PT-2245 illustrated); $10^{\pi} 10$ d'Entrecasteaux River source below Mt LaPerouse, alt. 800 m, 15 Feb. 1988, A. Neboiss. All specimens in NMV.

In general appearance and size similar to the other species in the genus.

Length of forewing: $075-5.9 \mathrm{~mm}$; o 5.2-5.7 $\mathrm{mm}$. The species is distinguished by details of the male genitalia.

\section{Diagnosis}

Ventral portion of segment IX rather robust, extended distally, with flat, laterally compressed, dorso-mesal projection; inferior appendages with harpago somewhat obscured by extended coxopodite; pre-anal appendages small; superior appendages (termed 'filipodes' by Schmid 1989) about $2 / 3$ length of segment $X$; parapodes elongate triangular with distal angle extended into laterally curved process. This is the most important distinguishing character separating this species from $P$. lepnevae.

Female abdomen with terminal segments somewhat flattened dorso-ventrally (Fig. 3A, B).

\section{Etymology}

Anastema (Gr) - height, in reference to elevation of the type locality.

\section{Distribution}

Known only from the type locality. Tasmanian endemic.

\section{Genus Ethochorema Neboiss}

Ethochorema Neboiss, 1977: 24.

Type species

Taschorema nesydrion Neboiss, 1962 by original designation.

\section{Ethochorema kelion Neboiss}

Ethochorema kelion Neboiss, 1977: 26.

Holotype

O Tasmania, Condominion Creek, 15 Feb. 1971 (NMV). Originally this species was recorded from only two localities, but recent collections show that it is more widespread than thought previously. All localities are from the SW and NW provinces.

\section{Additional material examined}

$10^{\pi}$ Gordon River, creek $500 \mathrm{~m}$ below Second Split, 12 Jan. 1977, A. Neboiss et al:; $10^{\star}$ Gordon River, $1 \mathrm{~km}$ above First Split, 11 Jan. 1977, A. Neboiss et al:; 1 o Condominion Creek, 12 Feb. 1980, K. Fairey; 1q same loc., 9 Feb. 1988, A. Neboiss and K. Walker; $30^{\star} 20$ Franklin River, Roaring Creek junction, $1 \mathrm{~km}$ above Gordon River, 8 Jan. 1977, A. Neboiss et al;; $10^{\star} 2$ Q Strathgordon, Jan. 1977, J. Sedlacek;

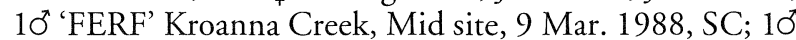
Lake Nancy, Frenchmans Cap area, 28 Jan. 1988, J. Jackson; $20^{\star} 5$ Q Lake Tahune, Frenchmans Cap area, 29 Jan. 1988, J. Jackson; 20 d'Entrecasteaux River source, below Mt La Perouse, alt. 840 m, 15 Feb. 1988, P.B. McQuillan. All specimens in NMV.

\section{Distribution}

Tasmania. Tasmanian endemic.

\section{Ethochorema secutum Neboiss}

Ethochorema secutum Neboiss, 1977: 24.

Holotype

đT Tasmania, 10 miles E of Strahan, 6 Feb. 1967; ANIC

\section{Additional localities}

Tasmania, 10 Ropeway Creek, $400 \mathrm{~m}$ below Smith and Gordon River Junction, 2 Feb. 1977, Colemman \& Richardson; $10^{\pi}$ Lake Pedder, Frankland shore campsite, 1 Mar. 1988, J. Jackson; $10^{\pi}$ Lake Pedder shore N of Forest Creek, 10 Feb. 1999, J. Jackson. All specimens in NMV. 


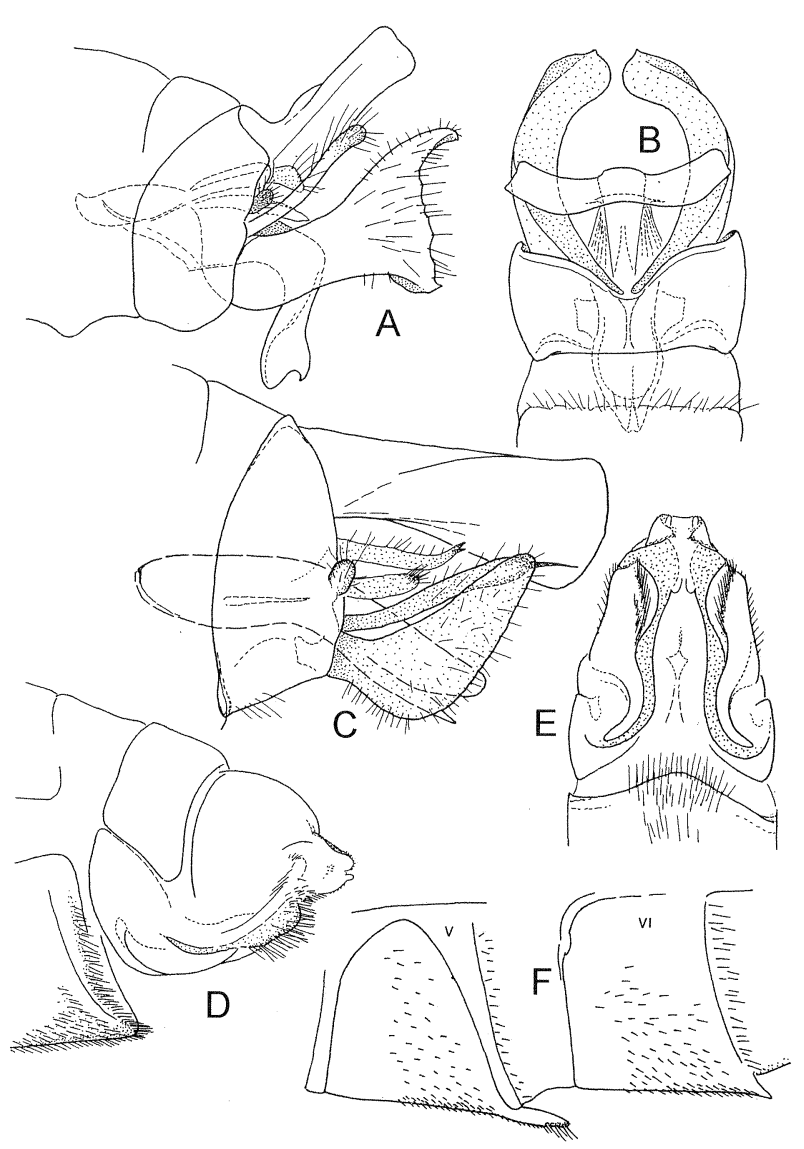

FIG. 5 - Taschorema $\operatorname{spp}$. (A, B) Taschorema dispatens sp. n., male genitalia: (A) lateral; (B) ventral; (C) T. ithyphallicum, male genitalia lateral; (D-F) T. pedunculatum female genitalia: $(D)$ lateral; $(E)$ ventral; $(F)$ abdominal sternites $V$ and VI lateral.

\section{Distribution}

Tasmania, NW and SW provinces. Tasmanian endemic.

\section{Genus Taschorema Mosely}

Taschorema Mosely, 1936: 422.

\section{Type species}

Taschorema asmana Moseley, 1936 by original designation.

\section{Taschorema dispatens sp. $\mathrm{n}$. Fig. $5 \mathrm{~A}, \mathrm{~B}$}

\section{Material examined}

Holotype $0^{\top}$ Tasmania, LaPerouse, source of d'Entrecasteaux River, alt. 840 m, 15 Feb. 1988, P.B. McQuillan and A. Neboiss (genitalia prep. PT-1699 illustrated), NMV.

\section{Length of forewing: $0^{\pi} 7.5 \mathrm{~mm}$.}

A medium-sized species, identified by the sessile fork 1 in hind wing and a total absence of ventral processes on abdominal segments. The strongly developed inferior appendages and the absence of two long bristles on the anal margin of forewing place this species close to Taschorema evansi Mosely.
Male genitalia (fig. 5A, B) characterised by large, rather robust, curved inferior appendages, which in lateral view are expanded and truncate apically; segment $\mathrm{X}$ elongate triangular in dorsal view; pre-anal appendages very short, superior appendages shorter than inferior appendages; two pairs of processes are located, mesally of pre-anal appendages (termed 'parapodes' by Schmid 1989). The phallus is robust, apically divided into two large down-turned lateral lobes.

Female unknown.

\section{Etymology}

Dispatens $(\mathrm{L})$ - opening in different directions, in reference to the shape of phallus.

\section{Distribution}

Tasmania, SW province. Known only from type locality. Tasmanian endemic.

\section{Taschorema ithyphallicum (Schmid) comb. nov.} Fig. 5C

Ethochorema ithyphallicum Schmid, 1989: 132; PI. XI figs 4-6.

\section{Material examined}

Holotype $0^{*}$ Tasmania, Franklin River Roaring Creek junction, 1 km above Gordon River, 8 Jan. 1977, D.Coleman, A.Neboiss, P.Allbrook. Paratype $10^{\star}$ Strathgordon, Jan. 1977, J. Sedlacek, NMV.

\section{Additional material examined}

$10^{\top}$ Lake Tahune, Frenchmans Cap area, 28 Jan. 1988, J. Jackson (male genitalia prep. PT-1698 illustrated).

Described by Schmid (1989), this species was placed in the genus Ethochorema close to secutum Neboiss. However, Schmid noted that the wing venation is close to that in Taschorema evansi Mosely. The absence of cross-vein C-Sc also agrees with the genus Taschorema. The wing venation is the major separating character between Taschorema and Ethochorema (Neboiss 1977). The male genitalic characters are variable in both genera and can not be used for generic identification. The species ithypallicum is therefore transferred to the genus Taschorema.

\section{Diagnosis}

Forewing ơ 9.3-9.5 mm; @ not known. Fork 1 long, sessile, fork 4 long; anal margin without two long bristles. Hind wing with cell-like structure between $\mathrm{Cu} 2$ and $\mathrm{Al}$; androconia present on $\mathrm{A} 2$ and $\mathrm{A} 3$.

Male genitalia (fig. 5C) segment X large, rather robust. Inferior appendages in lateral view somewhat triangular, pre-anal appendages small, rounded; superior appendages slender, about as long as inferior appendages with strong apical spine; two pairs of processes mesally (parapodes of Schmid), the upper one more sclerotised than the lower and terminating in a bipointed apex; the lower one semimembranous, shorter and rounded apically. Phallus slightly bent downwards at middle.

\section{Distribution}

All known localities are within the Southwest World Heritage Area. Tasmanian endemic. 


\section{Taschorema pedunculatum Jacquemart Fig. 5D-F}

Taschorema pedunculata Jacquemart, 1965: 35; Neboiss 1977: 30 .

\section{Holotype}

O Tasmania. Cradle Mountain, 12 Jan. 1923 (IRScNB).

\section{Diagnosis of female}

Abdomen (fig. $5 \mathrm{~F}$ ) with ridge on sternite $\mathrm{V}$ terminating at the lateral margin, ventrally developed into moderately large mesal process; a small pointed process on sternite VI; posterior margin of sternite VII covered with fine dense indistinct band of hair; segment VIII short, which together with part of segment IX forms elongate, rounded lateral pockets, visible in ventral view.

\section{Additional localities}

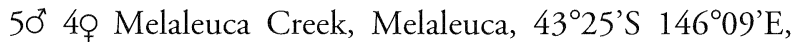
MV-light, 12 Feb. 1988, A. Neboiss and K. Walker (female genitalia prep. PT-2255 illustrated); $10^{*}$ Lake Pedder, shore $\mathrm{N}$ of Forest Creek, 10 Feb. 1999, J. Jackson; 20 Lake Pedder, 1 Mar. 1988, J. Jackson; 20 1 \% Lake Pedder, Frankland shore, 10 Feb. 1999, J. Jackson; $10^{7} 30$ Lake Pedder, Pebbly Creek south branch, 2 Mar. 1988, J. Jackson; $20^{\star}$ same loc. 10 Feb. 1999, J. Jackson; 30 below Maxwell Ridge, small tributary to Picton River, above Reservoir Lakes, 15 Feb. 1988, A. Neboiss; 70³ 39 d'Entrecasteux River source below Mt La Perouse, alt. 800 m, 16 Feb. 1988, A. Neboiss; $10^{\top} 2$ Q Ray River, 10 Feb. 1988, SC/PH; $10^{\circ}$ Gordon River $1 \mathrm{~km}$ above First Split, 11 Jan. 1977, A. Neboiss; $10^{\star}$ Olga-Hardwood River saddle, buttongrass plain, 4 Mar. 1977, P. Allbrook; $10^{\top}$ Dozer Creek below Gordon road, 4 Mar. 1999, J. Jackson; $10^{*}$ Franklin River Roaring Creek junction, $1 \mathrm{~km}$ above Gordon River, 8 Jan. 1977, A. Neboiss. All specimens in NMV.

\section{Remarks}

The species, previously known from very few localities, is now recorded quite widely from the SW province. It is also the first time that the female has been associated positively with the male.

\section{Distribution}

Tasmania. Tasmanian endemic.

\section{Taschorema viridarium Neboiss}

Taschorema.viridarium Neboiss, 1962: 563; Neboiss 1977: 29.

\section{Holotype}

OT Tasmania, Broad River nr Lake Dobson, Mt Field National Park, 6 Nov. 1955 (NMV).

\section{Additional localities}

$50^{\star} 12$ Q Doctors Creek at Lake Highway near Breona, 9 Feb. 2001, R.Walker; I0 Pinetree Rivulet nr Breona, 9 Feb. 2001, R. Walker; $10^{\top} 1$ \% Sandfly Creek at Scotts Peak road, 9 Feb. 1988, K.Walker and J. Jackson; $0^{\top}$ o (numerous) d'Entrecasteaux River, South Coast road bridge, 14 Feb. 1988, A. Neboiss and K.Walker. 30 Old River and Collingwood River junction, 10 Feb. 1988, A. Neboiss et al.; lo Navarre River at Lyell highway, 3 Nov. 1998, J. Jackson; $10^{\top}$ Collingwood River below Lyell Highway bridge, 16 Feb. 1999, J. Jackson.

\section{Distribution}

Tasmania, widespread. Tasmanian endemic.

\section{GenusPtychobiosis Neboiss}

Ptychobiosis Neboiss, 1977: 32.

\section{Type species}

Notiobiosis nigrita Banks, 1939 by original designation.

In recent years additional material and detailed studies of adults and larvae have revealed that Ptychobiosis nigrita (Banks) s.l. represents a species complex which is distributed from North Queensland (type locality Mt Spurgeon) along eastern Australia to Victoria and Tasmania. The small differences found in Tasmanian specimens by Neboiss (1977) were not regarded of sufficient importance for species separation. Additional information is now available and is supported by distinctive larval features as shown by Dean (1997). The Tasmanian population referred previously to the nigrita complex is now regarded as a distinct species.

\section{Ptychobiosis iconica sp. $\mathrm{n}$. Fig. 6A-C}

\section{Type material}

Holotype $0^{\star}$ Tasmania, Olga-Hardwood River saddle, buttongrass plain, 4 Mar. 1977, P. Allbrook, J. Richardson and R. Swain. Paratypes $70^{\circ} 10$ collected with holotype (female genitalia prep. PT-2244 illustrated); $120^{*}$ Strathgordon, Jan. 1977, J. Sedlacek (male genitalia prep. PT-2177 illustrated); lo Sandfly Creek at Scotts Peak road, 9 Feb. 1988, K. Walker, J. Jackson; $50^{\star}$ E Arthur Ranges, $1 \mathrm{~km}$ W of Federation Peak, alt.1000 m, 11 Feb. 1988, P. McQuillan; 10 Lake Pedder, shore N of Forest Creek, 10 Feb. 1999, J. Jackson; 10 Lake Pedder, S of Timber Creek, 10 Feb. 1999, J. Jackson; 2Q Lake Pedder, Teds Beach S corner, 4 Mar. 1999, J. Jackson; $20^{\circ}$ Condominion Creek at Scotts Peak road; 9 Feb. 1988, A. Neboiss, K. Walker; $30^{\star}$ Condominion Creek, 12 Feb. 1980, K. Fairy. All specimens in NMV.

\section{Additional material examined}

$20^{\circ}$ Cardigan River above Lyell Highway bridge, 16 Feb. 1999, J. Jackson; $40^{*}$ Nelson River below Raglan Road bridge, 16 Feb. 1999, J. Jackson; 10 1o Lake Tahune, Frenchmans Cap area, 28 Jan. 1988, J.Jackson; 1ㅇ Picton River tributary above Reservoir Lake, 43⒉' $146^{\circ} 441^{\prime} \mathrm{E}$, alt. 800 m, 15 Feb. 1988, A. Neboiss; $30^{\circ}$ Melaleuca Creek near Melaleuca, 432 25'S 1460'ㄹ, 12 Feb. 1988, A. Neboiss, K. Walker; $20^{\circ} 10$ Hartz Mountains, Jan. 1977, J. Sedlacek; $30^{\pi}$ d'Entrecasteaux River source, below Mt La Perouse, alt. 840 m, 15 Feb. 1988, P.B. McQuillan; 1 s same. loc. 16 Feb. 1988, A. Neboiss; 50 1\% New River Lagoon, buttongrass, 15 Feb. 1988, J. Jackson; 20 Serpentine impoundment, Scotts Peak Dam, 23 Mar. 1985, P. Greenslade (NMV).

All Tasmanian specimens previously identified as Ptychobiosis nigrita (Banks) are now referred to Ptychobiosis iconica. 


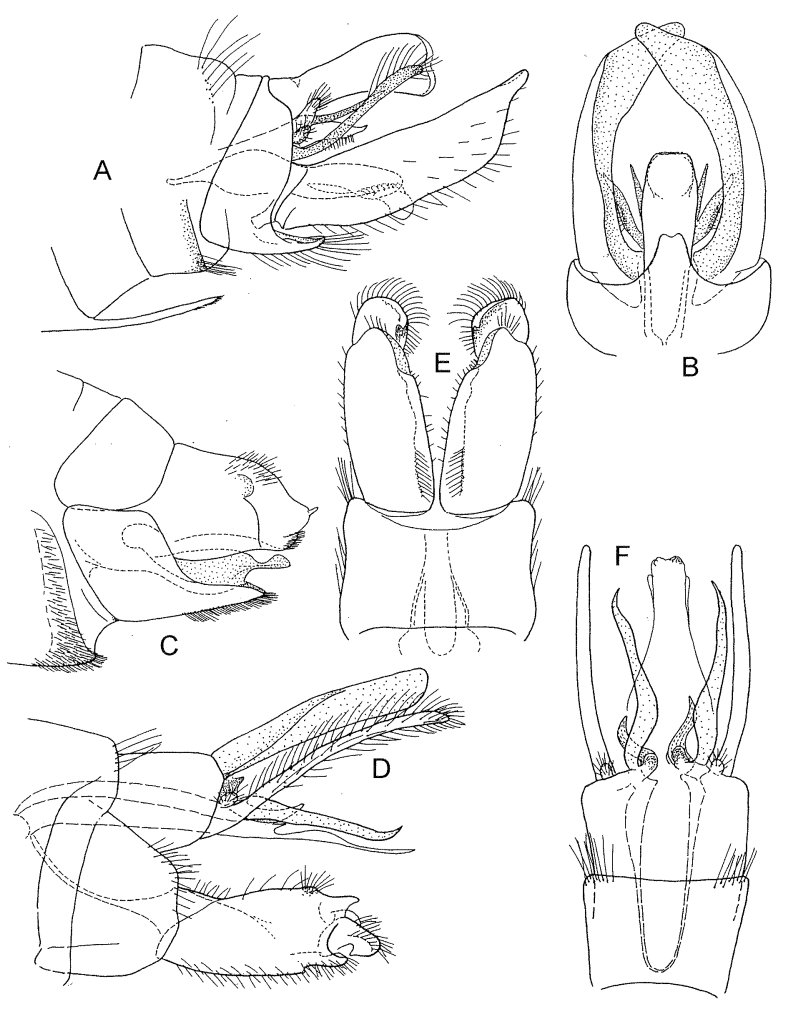

FIG. $6-(A-C)$ Ptychobiosis iconica sp. n.: (A) male genitalia lateral; $(B)$ ventral; $(C)$ female genitalia lateral; (D-F) Moruya tasmanica male genitalia: (D) lateral; (E) ventral; $(F)$ dorsal.

\section{Diagnosis}

Male genitalia (fig. 6A, B), segment $\mathrm{X}$ rather robust, the inferior appendages in ventral view curved inward, rounded apically; parapodes ( $=$ term used by Schmid 1989 , upper penis cover used by Mosely \& Kimmins 1953) with outer branch short, rounded; meso-ventral process of segment IX rather short, not elevated distally, much narrower than in nigrita.

Female genitalia (fig. 6C) sternite $\mathrm{V}$ with strong ventral process, sternite VI with ventral process small; sternite VII lacking process; tergite rather small, but sternite extended distally into apically rounded lip, distal end densely pubescent - in nigrita the lip is longer, narrower and apically truncate; terminal segment ends into two rounded lobes.

\section{Etymology}

Icon $(\mathrm{L})$ - image, in reference to the resemblance to nigrita.

\section{Distribution}

Throughout the state of Tasmania, but more common in the western provinces. Tasmanian endemic.

\section{Genus Koetonga Neboiss}

Koetonga Neboiss, 1962: 567.

\section{Type species}

Koetonga clivicola Neboiss, 1962, by original designation.

\section{Koetonga clivicola Neboiss}

Koetonga clivicola Neboiss, 1962: 567; Neboiss 1977: 33; Schmid 1989: 81.

\section{Holotype}

ơ Victoria, Timbertop nr Merrijig, 5 June 1958 (ANIC).

\section{Additional localities}

Tasmania, 10 Sir John Falls, Cataract Creek, 9 Jan. 1977, A. Neboiss; $10^{\star}$ Dozer Creek below Gordon road, 4 Mar. 1999, J. Jackson; 70’ 2Q Olga River, $19 \mathrm{~km}$ above Gordon River junction, 13 Jan. 1977, D. Coleman and P. Allbrook; 19 Wedge River above Gordon road (in forest), 26 Jan. 1999, J. Jackson; 50 1o Sandfly Creek at Scotts Peak road, 9 Feb. 1988, K. Walker and J. Jackson; $10^{\top}$ Doctors Creek at Lake Highway nr Breona, 9 Feb. 2001, R. Walker; $30^{*}$ Port Arthur, Jan. 1977, J. Sedlacek; $20^{\star} 10$ d'Entrecasteaux River at South Coast road bridge, 14 Feb 1988, A. Neboiss and K. Walker. All specimens in NMV.

\section{Distribution}

Tasmania, widespread. Other states: Victoria and New South Wales.

\section{Genus Moruya Neboiss}

Moruya Neboiss, 1962: 569.

\section{Type species}

Moruya charadra Neboiss, 1962 by original designation.

This endemic Tasmanian genus is represented by three closely related species. New figures are provided here for the male of Moruya tasmanica Jacquemart from recently collected material; the original illustrations did not show all the necessary details for positive identification. For the first time females of all three species are identified positively and a key for their separation is provided. Unfortunately, for critical separation of charadra and tasmanica it is still necessary to clear the abdomen to allow the view of vaginal sclerites.

\section{Key to males of Tasmanian Moruya}

1. Processes arising at the base of segment X asymmetric, shorter on one side .................. charadra Processes arising at base of segment $\mathrm{X}$ the same length on both sides 2

2. The short upper processes dorsally with small rounded projection at base; the long lower processes turned upward apically ..... The short upper processes two-branched, of similar length; the long lower processes curved downward apically opora

Key to females of Tasmanian Moruya

1. Abdominal cerci pigmented, dark.. Cerci not pigmented (fig. $7 \mathrm{H}$ ) 2

2. Vaginal sclerite curved (fig. 7D) charadra Vaginal sclerite robust, L-shaped (fig. 7A) .... tasmanica 


\section{Moruya charadra Neboiss} Fig. 7D-7F

Moruya charadra Neboiss, 1962: 570; Neboiss 1977: 34; Schmid 1989: 74.

\section{Holotype}

o Tasmania, Broad River nr Lake Dobson, 6 Nov. 1955 (QM).

\section{Additional material examined}

Tasmania, 200 30o Old River and Collins River junction, $43^{\circ} 16^{\prime}$ S, $146^{\circ} 20^{\prime} \mathrm{E}, 10$ Feb. 1988 , A. Neboiss et al. (female genitalia prep. PT-2165 illustrated); $20^{\star} 10$ d'Entrecasteau River source, below Mt LaPerouse, 4330'S 146 44'E, alt. 840 m, 16 Feb. 1988, A. Neboiss and K. Walker; $10^{\star} 30$ Wedge River above Gordon road, 26 Jan. 1999, J. Jackson; $150^{\circ}$ o Melaleuca Creek, near Melaleuca, $43^{\circ} 25^{\prime} \mathrm{S}, 146^{\circ} 09^{\prime} \mathrm{E}$, 12 Feb. 1988, A. Neboiss and K. Walker; 10 Dozer Creek below Gordon road, 4 Mar. 1999, J. Jackson; 10 below Maxwell Ridge, tributary of Picton River above Reservoir Lakes, $43^{\circ} 29^{\prime} \mathrm{S}, 146^{\circ} 44^{\prime} \mathrm{E}, 15$ Feb. 1988, A. Neboiss; 19 Huon River below Picton River junction, 1 Feb. 1980, I. Campbell; 1o Hartz Creek, Hartz Mountains National Park, 25 Apr. 1974 (no collector); 10'10 Navarre River at Lyell Hway, 3 Nov. 1998, J. Jackson; 1Q Collingwood River below Lyell Highway bridge, 3 Nov. 1998, J. Jackson; 20 1우 Douglas Creek High Bridge near Old Pelion Hut, Cradle Mountain-Lake St Clair National Park, 18 Jan. 1990, J. Dean and D. Cartwright; $10^{*}$ Gordon River $2 \mathrm{~km}$ below Serpentine River junction, 12 Jan. 1977, A. Neboiss; $10^{*}$ Gordon River, $1 \mathrm{~km}$ above First Split, 11 Jan. 1977, A. Neboiss.

Female genitalia (fig. 7D-F) characterised by pigmented cerci and vaginal sclerite gradually curved. The sclerite configuration differs from that of $M$. tasmanica by the curved upper outline visible in cleared preparation.

\section{Distribution}

Tasmania, widespread, particularly throughout western parts of the state. Tasmanian endemic.

\section{Moruya tasmanica (Jacquemart) Figs $6 \mathrm{D}-7 \mathrm{C}$}

Psyllobetina tasmanica Jacquemart, 1965: 45. Moruya tasmanica. Neboiss 1977: 35.

\section{Holotype}

o Tasmania, Cradle Mountain, 27 Jan. 1923 (IRScNB).

\section{Additional material examined}

Tasmania 50 40 Lake Tahune, Frenchmans Cap area, 29 Jan. 1988, J. Jackson (NMV). (Male and female genitalia preps PT-2166 O', PT-2167 o illustrated.)

Male genitalia (fig. 6D-F) segment $\mathrm{X}$ and superior appendages about the same length and about as long as, or slightly longer than, the inferior appendages (they appear shorter in the original drawing). Small, rounded pre-anal appendages are located at the base of superior appendages. At the distal margin of tergite IX and just below segment
$\mathrm{X}$ arise two pairs of processes (= parapodes as used by Schmid 1989), the upper process short, with dorsally elevated, rounded projection at base, twisted, unequal in length; the lower processes long, terminating with upturned, pointed apices. Inferior appendages robust, coxopodite with elongate group of strong spines on the mesal ridge near the base, harpago short with distinct curved setae apically.

Female genitalia (fig. 7A-C) with cerci pigmented, similar to those of $M$. charadra; the distal end of ovipositor appears to be somewhat more robust than in charadra; the vaginal sclerite in lateral view distinctly L-shaped and rather robust.

\section{Distribution}

Tasmania, restricted, known from only a few localities in C and NW provinces. Tasmanian endemic.

\section{Moruya opora Neboiss Fig. 7G-I}

Moruya opora Neboiss 1962: 571; Neboiss 1977: 34.

\section{Holotype}

ơ Tasmania, Ouse River nr Great Lake, 2 Apr. 1960 (ANIC).

\section{Additional material examined}

Tasmania, 7o Lake Pedder, Serpentine boat ramp, 4 Mar. 1999, J. Jackson; 3o Lake Pedder, shore N of Forest Creek, 10 Feb. 1999, J. Jackson; 10 Lake Pedder, Teds beach S corner, 4 Mar. 1999, J. Jackson; 30 10ㅇ Franklin River above Lyell Highway bridge, 16 Feb. 1999, J. Jackson; 10 Nelson River below Raglan road bridge, 16 Feb. 1999, J. Jackson; lo Collingwood River below Lyell Highway bridge, 3 Nov. 1998, J. Jackson; lo, same loc., 16 Feb. 1999, J. Jackson; 4O Wedge River above Gordon road, 26 Jan. 1999, J. Jackson; 10 Huon-Picton River junction, 18 Feb. 1967, A. Neboiss (female genitalia prep. PT-2171 illustrated); $20^{\circ}$ 20 Old River and Collins River junction, $43^{\circ} 16^{\prime} \mathrm{S} 146^{\circ} 20^{\prime} \mathrm{E}$, 10 Feb. 1988, A. Neboiss et al.; lo d'Entrecasteaux River, South Cape road bridge, 14 Feb. 1988, A. Neboiss and K. Walker. All specimens in NMV.

Females of this species can be separated from the other two Tasmanian species on the basis of genitalic characteristics, namely the unpigmented cerci and basally broad vaginal sclerite (fig. 7I).

\section{Distribution}

Tasmania, widespread. Tasmanian endemic.

\section{FAMILY HYDROPTILIDAE}

The family Hydroptilidae in Australia has been extensively revised by Wells in a series of papers published between 1979 and 1998. Taxonomic problems were discussed, new species described and information given on species' distributions. Seven genera and a total of 21 species are now recorded from the island. 


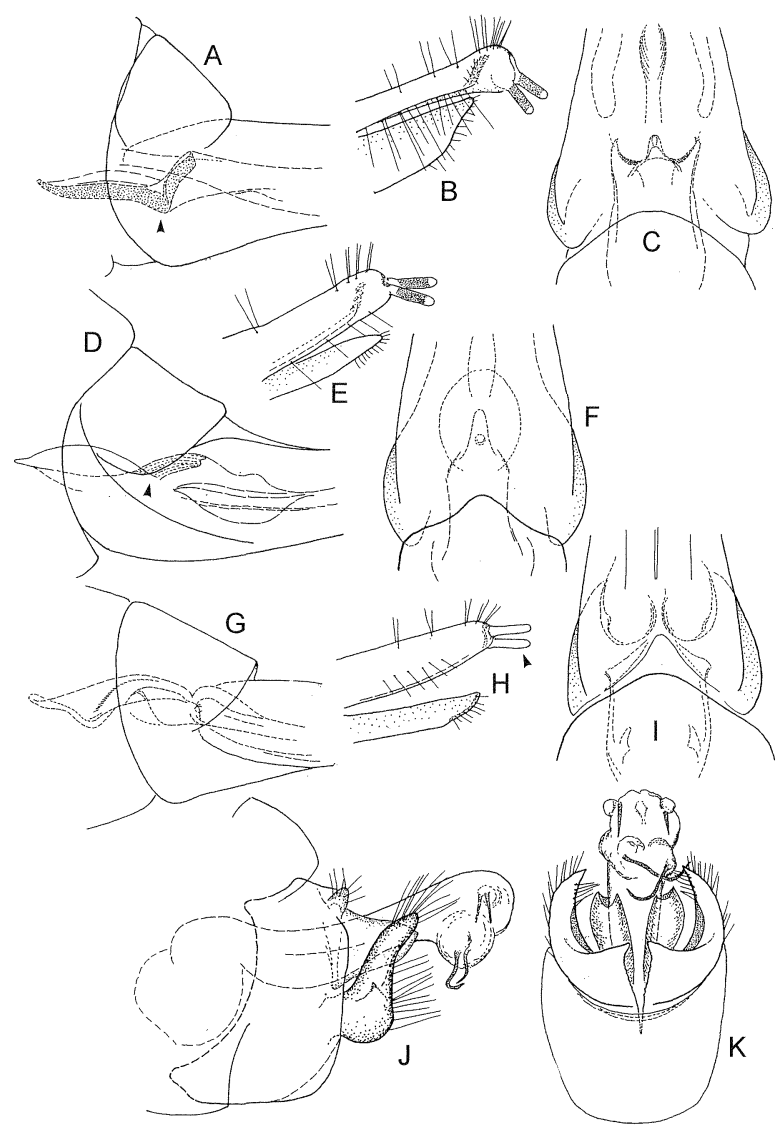

FIG. $7-(A-I)$ Moruya spp.: $(A-C)$ Moruya tasmanica, female genitalia: (A) lateral; (B) tip of ovipositor; $(C)$ ventral. (D-F) M. charadra, female genitalia: (D) lateral; (E) tip of ovipositor; $(F)$ ventral. $(G-I)$ M. opora, female genitalia: $(G)$ lateral; (H) tip of ovipositor; (I) ventral; $(J, K)$ Chimarra derogata sp. n., male genitalia: (J) lateral; $(K)$ ventral.

\section{Genus Hydroptila Dalman}

Hydroptila Dalman, 1819; Wells 1978: 746.

Type species

Hydroptila tineoides Dalman, 1819 by monotypy.

\section{Hydroptila tasmanica Mosely}

Hydroptila tasmanica Mosely, 1934: 147; Mosely \& Kimmins 1953: 509; Neboiss 1977: 40; Wells 1978: 745.

Holotype

o Tasmania, Wilmot, 9 Jan. 1917 (BMNH).

Distribution

Tasmania - widespread; not recorded outside the state.
Holotype

OT Tasmania, Scamander River, Upper Scamander, 9 Nov. 1972 (NMV).

\section{Distribution}

Tasmania - South Esk River, Evandale. Other states: widespread in eastern Victoria, New South Wales and Queensland.

\section{Hydroptila acinacis Wells}

Hydroptila acinacis Wells, 1978: 755; Wells 1985: 6 (larva).

\section{Holotype}

ot Victoria, Koornalla, Traralgon Creek, 26 Feb. 1974 (NMV).

\section{Distribution}

Tasmania - Forth Falls; Derwent River trib. nr Gretna; Guide River Falls near Ridgley; Hellyer River Gorge. Other states: Victoria and New South Wales.

\section{Genus Orphninotrichia Mosely}

Orphninotrichia Mosely, 1934; Wells 1980: 628.

Type species

Orphnionotrichia maculata Mosely, 1934 by original designation.

\section{Orphninotrichia maculata Mosely}

Orphninotrichia maculata Mosely, 1934: 139; Wells 1980: 630; Wells 1985: 21 (larva).

Holotype

ऽ๋ New South Wales, Hornsby, 31 Aug. 1916 (BMNH).

\section{Distribution}

Tasmania - Wedge River; Gelignite Creek at Scotts Peak Dam road. Other states: Victoria, South Australia, New South Wales, Queensland.

\section{Orphninotrichia acta Neboiss}

Orphninotrichia acta Neboiss, 1977: 40; Wells 1980: 632.

Holotype

Ơ Tasmania, Ulverstone, 18 Nov. 1972 (NMV).

\section{Distribution}

Tasmania - Forth Falls; Iris River tributary at Middlesex Plains; Broken Leg Creek at Lyell Highway. Tasmanian endemic.

\section{Genus Maydenoptila Neboiss}

Maydenoptila Neboiss, 1977: 44; Wells 1980: 635.

Hydroptila scamandra Neboiss, 1977: 41; Wells 1978: 751; Wells 1985: 5 (larva). 
Type species

Maydenoptila cuneola Neboiss, 1977 by original designation.

\section{Maydenoptila cuneola Neboiss}

Maydenoptila cuneola Neboiss, 1977: 44; Wells 1980: 637; Wells 1985: 25 (larva).

Holotype

ơ Tasmania, Wedge River, 17 Feb. 1971 (NMV).

Distribution

Tasmania - Gordon River First Split; Murchison River; Maxwell-Denison River junction; d'Entercasteaux River; Adams Falls; Old River-Collins River junction. Other states: Victoria

\section{Maydenoptila explicata Wells}

Maydenoptila explica Wells, 1980: 639.

\section{Holotype}

OT Tasmania - Gordon River, $1 \mathrm{~km}$ above First Split, 11 Jan. 1977 (NMV).

\section{Distribution}

Tasmania — Gordon River at Sprent junction; Russell Falls; Fern Creek near Rosebery; Murchison River. Not recorded outside Tasmania.

\section{Maydenoptila rupina Neboiss}

Maydenoptila rupina Neboiss, 1977: 45; Wells 1980: 643; Wells 1985: 24 (larva).

\section{Holotype}

OT Tasmania — Guide River Falls nr Ridgley, 18 Nov. 1972 (NMV).

Distribution

Tasmania - Scamander River, Upper Scamander; Russell Falls. Other States: Victoria, South Australia.

\section{Genus Tricholeiochiton Kloet \& Hincks}

Tricholeiochiton Kloet \& Hincks, 1944: 97; Wells 1982: 252.

Type species

Leiochiton fagesii Guinard, 1878 by monotypy.

\section{Tricholeiochiton pennyae Wells}

Trichoeliochiton pennyae Wells, 1998: 81.

Holotype

O Tasmania - World Heritage Area, Melaleuca, 13. Feb. 1997 (ANIC).
Distribution

Tasmania - World Heritage Area, Mary Ann Creek; King Willian Creek Site; Lake St Clair; McPartlan Pass; Airstrip Road. Tasmanian endemic.

\section{Genus Hellyethira Neboiss}

Hellyethira Neboiss, 1977: 42; Wells 1979: 314.

Type species

Hellyethira vallecula Neboiss, 1977 (=Xuthotrichia simplex Mosely, 1934) by original designation.

\section{Hellyethira simplex (Mosely)}

Xuthotrichia simplex Mosely, 1934: 145.

Hellyethira vallecula Neboiss, 1977: 42.

Hellyethira simplex, Wells 1979:315; Wells 1985: 10 (larva).

Holotype

Oั Queensland - Warwick, October (BMNH). Holotype of $H$. vallecula $\sigma^{*}$ Tasmania, Hellyer River Gorge, 9 Feb. 1971 (NMV).

\section{Distribution}

Tasmania - E, N, NW and SW provinces. Other states: disjunct distribution ranges from northern Queensland along Great Dividing Range from northern NSW throughout Victoria, Kangaroo Island and South Australia; according to Wells (1979), this is the most abundant and widespread hydroptilid in eastern Australia.

\section{Hellyethira basilobata Wells}

Hellyethira basilobata Wells, 1979: 316; Wells 1985: 11 (larva).

Holotype

○’ Victoria, Yarra River below Upper Yarra Dam, 28 Feb. 1976 (NMV).

\section{Distribution}

Tasmania - Dip River Falls; Boyd River, Gordon road; Cardigan River at Lyell Highway; Navarre River at Lyell highway. Other states: Victoria - eastern, north-central and Otway Ranges.

\section{Hellyethira exserta Wells}

Hellyethira exserta Wells, 1979: 319; Wells 1985: 11 (larva).

Holotype

o New South Wales, Boonoo Boonoo River, $5 \mathrm{~km}$ upstream from falls, 11 Oct. 1973 (NMV).

\section{Distribution}

Tasmania - South Esk River, Evandale; Huon-Picton River junction; Sir John Falls. Other states: Victoria, South Australia. 


\section{Hellyethira malleoforma Wells}

Hellyethira malleoforma Wells, 1979: 325; Wells 1985: 13 (larva).

Holotype

ơ South Australia, Uraidla, 28 Nov. 1975 (ANIC).

Distribution

Tasmania - N of Gretna. Other states: scattered throughout eastern Australia from Cairns to Birdsville in Queensland, to Bourke, NSW, Victoria and southern South Australia.

\section{Hellyethira multilobata Wells}

Hellyethira multilobata Wells, 1979: 326.

Holotype

ơ Victoria, Lake Purrumbete, 23 Feb. 1970 (ANIC).

\section{Distribution}

Tasmania - South Esk River, Evandale; Hellyer River Gorge; Lake Pedder, Teds Beach, Forest Creek and Maria Creek; Olga River; Margate. Other states: Victoria, South Australia, New South Wales.

\section{Genus Oxyethira Eaton}

Oxyethira Eaton, 1973: 143; Wells 1981: 104.

Type species

Hydroptila flavicornisPictet, 1834 (= Oxyethira costalisEaton, 1873) by original designation.

\section{Oxyethira subgenus}

\section{Trichoglene Neboiss}

Trichoglene Neboiss, 1977: 43.

Type species

Trichoglene columba Neboiss, 1977 by original designation.

\section{Oxyethira (Trichoglene) columba (Neboiss)}

Trichoglene columba Neboiss, 1977: 43. Oxyethira columbaWells 1981: 160; Wells 1985: 17 (larva). Oxyethira (Trichoglene) Wells 1987: 133.

\section{Holotype}

o Tasmania - Dove River, Cradle Mountain National Park, 14 Dec. 1972 (NMV).

Distribution

Tasmania - widespread throughout the state. Other states: Central Victoria, scattered localities in South Australia, New South Wales and Queensland.

\section{Oxyethira (Trichoglene) mienica Wells}

Oxyethira mienica Wells, 1981: 108.
Holotype

ơ Tasmania - Ouse River, 5 miles W of Miena, 28 Feb. 1967 (ANIC).

Distribution

Tasmania - restricted and rare. Not recorded outside Tasmania.

\section{Oxyethira (Trichoglene) tasmaniensis Wells}

Oxyethira tasmaniensis Wells, 1998: 83.

Holotype

○ Tasmania - World Heritage Area, Melaleuca, 13 Feb. 1997 (ANIC).

Distribution

Tasmania - restricted and rare. Not recorded outside Tasmania.

\section{Oxyethira (Trichoglene) driesseni Wells, 2002}

Oxyethira (Trichoglene) driesseni Wells, 2002: 39.

Holotype

Ơ Tasmania, Lake St Clair, Site RCE young, 17 Jan. 2000, ANIC.

Distribution

Tasmania, C province. Known only from type locality. Tasmanian endemic.

\section{Oxyethira (Trichoglene) torquata Wells, 2002}

Oxyethira (Trichoglene) torquata Wells, 2002: 39.

\section{Material examined}

Holotype o Tasmania, McPartlan Pass, Site 8A, 7 Mar. 2000, M. Driessen, ANIC.

\section{Distribution}

Tasmania, C province. Known only from type locality Tasmanian endemic.

\section{Genus Orthotrichia Eaton}

Orthotrichia Eaton, 1873: 141; Wells 1979: 586.

Type species

Hydroptila angustella McLachlan, 1865 by original designation.

\section{Orthotrichia zonata (Neboiss)}

Targatrichia zonata Neboiss, 1977: 41.

Orthotrichia zonata. Wells 1979: 591.

Holotype

OT Tasmania, St Patricks River, Targa, 22 Feb. 1971 (NMV). 
Distribution

Tasmania - widespread throughout the state. Tasmanian endemic.

\section{Orthotrichia adornata Wells}

Orthotrichia adornata Wells, 1979: 590.

\section{Holotype}

OT Victoria, Millgrove, Yarra River, 24 Feb. 1976 (NMV).

Distribution

Tasmania - Bushy Park. Other states: Victoria, New South Wales.

\section{FAMILY PHILOPOTAMIDAE}

The family Philopotamidae is represented in mainland Australia by two genera, both of which are now also recorded from Tasmania. The genus Chimarra Stephens is here recorded from Tasmania for the first time and only from a single species. The genus Hydrobiosella Tillyard is widely distributed throughout the state. The species limitations in some cases are rather unclear and difficult to interpret.

\section{Genus Chimarra Stephens}

Chimarra Stephens, 1829: 318.

\section{Type species}

Phryganea marginata Linnaeus, 1767 by monotypy.

The genus Chimarra is well known from continental Australia from four described and a number of undescribed (now under review) species. It is here recorded for the first time from Tasmania. The only known specimen, here described as a new species, has been known for some 40 years, but its description was deferred with hopes to locate more specimens, but so far without success. The type locality near the northern end of the Great Lake was severely disturbed by road-making and repeated searches in the general area have been unsuccessful in locating further specimens.

\section{Chimarra derogata sp. $\mathrm{n}$. Fig. 7J, K}

\section{Type material}

Holotype $0^{*}$, Tasmania, Great Lake, small stream S of Breona, 27 Jan. 1960, F. McDonald (male genitalia prep. PT-802 illustrated), NMV.

Generally similar to Chimarra monticola Kimmins from Victoria and New South Wales, but $C$. derogata differs in details of male genitalia. The single alcohol-preserved specimen is faded and pale brownish colour, not showing any wing pattern.

Length of forewing: $O^{\star} 6.1 \mathrm{~mm}$. Spurs 1:4:4; male genitalia (Fig. 7J, K) segment IX short; superior appendages small, in lateral view elongate ovoid; segment $\mathrm{X}$ somewhat triangular in lateral view, only lightly sclerotised, upper margin not produced distally; inferior appendages short, abruptly curved upwards, meso-ventral angles acute triangular; phallus membranous, expanded distally, with two pairs of twisted spines internally.

\section{Distribution}

Tasmania - Central province, known from type locality only.

\section{Etymology}

Derogatus (L) - 'belittle' in reference to reduced size of segment $\mathrm{X}$.

\section{Genus Hydrobiosella Tillyard}

Hydrobiosella Tillyard, 1924: 288; Neboiss 1977: 45.

\section{Type species}

Hydrobiosella stenocerca Tillyard, 1924 by monotypy.

The genus is well represented in Tasmania with 15 species, placed in three species groups.

\section{corinna group — four species}

Characterised by very slender male genitalia, small but distinct superior appendages at the base of segment X; sternite and tergite IX both strongly sclerotised and fused; inferior appendages slender, harpago with small group of short dark setae on the inner surface, phallus elongate with sclerotised inner spines near apex.

\section{Species included}

Corinna Neboiss; orba Neboiss; anasina Neboiss and cerula Neboiss.

\section{tasmanica group — ten species}

Male genitalia somewhat stouter than in corinna group; superior appendages absent; sternite and tergite IX both strongly sclerotised and fused; inferior appendages, slender, harpago with small group of short, dark setae on the inner surface and a single curved bristle at the apex; phallus elongate with pair of sclerotised lateral bars and apical pair of downturned stout spines.

\section{Species included}

Tasmanica Mosely; anatolica sp. n.; armata Jacquemart; cognata Kimmins; sagitta Neboiss; propinquasp. n.; disrupta sp. n.; otaria sp.a.; scalaris sp. n.; tahunense sp. n.

\section{waddama group - one species}

Male genitalia rather more robust than in the other two groups; superior appendages absent; segment IX with tergite membranous, only sternite sclerotised, rather elongate; inferior appendages robust; phallus with pair of long curved processes.

\section{Species included}

Waddama Mosely. 
corinna group

\section{Hydrobiosella corinna Neboiss Figs $8 \mathrm{~A}, 8 \mathrm{~B}, 9 \mathrm{~A}$}

Hydrobiosella corinna Neboiss, 1977: 47.

Holotype

o Tasmania, Corinna (small creek in forest), 5 Nov. 1972 (NMV).

Additional material examined

Tasmania, $O^{\pi}$ (numerous) Franklin River-Roaring Creek junction, $1 \mathrm{~km}$ above Gordon River, 8 Jan. 1977, A.Neboiss (male genitalia prep. PT-2209 illustrated) NMV.

\section{Diagnosis}

Male genitalia (fig. 8A, B) superior appendages present, short, somewhat pointed apically; segment $\mathrm{X}$ in dorsal view rather robust elongate, somewhat triangular apically. Phallus membranous with one sclerotised spine about midway, and a pair of $\mathrm{V}$-shaped sclerites near distal end.

\section{Distribution}

Tasmania, NW and northern SW provinces. Tasmanian endemic.

\section{Hydrobiosella orba Neboiss Figs $8 \mathrm{C}, 8 \mathrm{D}, 9 \mathrm{~B}$}

Hydrobiosella orba Neboiss, 1977: 47.

Holotype

O' Tasmania, Wedge River, 17 Feb. 1971 (NMV).

\section{Additional localities}

Tasmania, $110^{\pi}$ Gordon River, $1 \mathrm{~km}$ above First Split, 11 Jan. 1977, A. Neboiss; $70^{\star}$ Gordon River, $500 \mathrm{~m}$ below Second Split, 12 Jan. 1977, A. Neboiss (male genitalia prep. PT-2194 illustrated); $10^{\pi}$ Ropeway Creek, $400 \mathrm{~m}$ below Smith and Gordon River junction, 2 Feb. 1977, D. Coleman; $10^{\pi}$ stream, $300 \mathrm{~m}$ W of Teds Beach turnoff, 14 Jan. 1999, J. Jackson; 10“ Lake Oberon, Western Arthur Ranges, top of plain, 9 Mar. 1999, J. Jackson. (All specimens in NMV.)

\section{Diagnosis}

Male genitalia (fig. 8C, D) superior appendages somewhat smaller than in corinna; segment $\mathrm{X}$ shorter, in dorsal view almost triangular. Phallus membranous with two pairs of strongly sclerotised $\mathrm{V}$-shaped sclerites, one pair about midway the other near distal end.

\section{Distribution}

Tasmania, SW province. Tasmanian endemic.

\section{Hyrobiosella anasina Neboiss Figs $8 \mathrm{E}, 8 \mathrm{~F}, 9 \mathrm{C}$}

Hydrobiosella anasina Neboiss 1977: 48.

\section{Holotype}

OT Tasmania, Duck River, 6 km SW of Roger River, 29 Nov. 1974 (NMV).

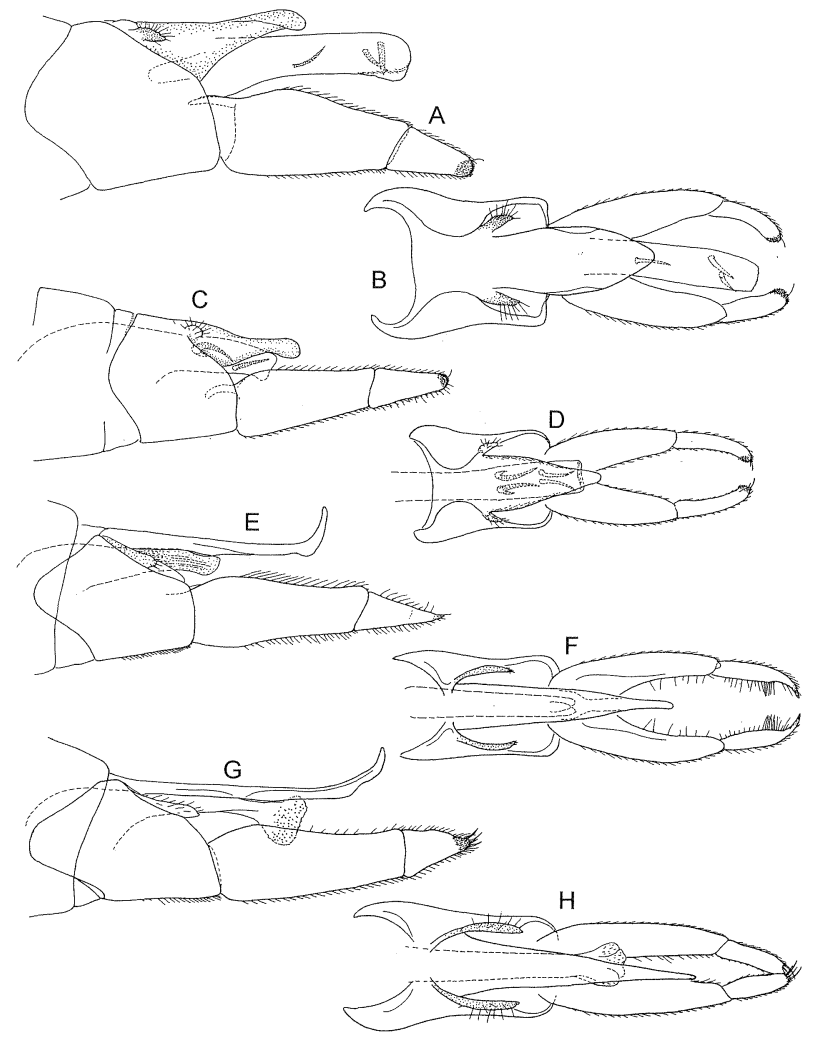

FIG. 8- Hydrobiosella spp., male genitalia. (A, B) $\mathrm{H}$. corinna: $(A)$ lateral; $(B)$ dorsal. $(C, D) \mathrm{H}$. orba: (C) lateral; (D) dorsal. $(E, F) \mathrm{H}$. anasina: (E) lateral; $(F)$ dorsal. $(G, H)$ H. cerula: $(G)$ lateral; $(H)$ dorsal.

\section{Additional localities}

Tasmania, 220 Gordon River, $1 \mathrm{~km}$ above First Split, 11 Jan. 1977, A. Neboiss (male genitalia prep. PT-2261 illustrated); $40^{*}$ Lake Tahune, Frenchmans Cap area, 29 Jan. 1988, J. Jackson. (NMV).

\section{Diagnosis}

Male genitalia (fig. 8E, F) superior appendages present; segment X long, slender, tapering distally, extends almost to the base of harpago, apex laterally somewhat compressed and more or less abruptly turned upward. Inferior appendages long, harpago less than half the length of coxite, in lateral view somewhat triangular, terminates with mesally directed acute point. Phallus membranous without internal sclerotised spines, several rows of fine spicules laterally at distal end.

\section{Distribution}

Tasmania - NW, Cand northern SW provinces. Tasmanian endemic.

\section{Hydrobiosella cerula Neboiss Figs $8 \mathrm{G}, 8 \mathrm{H}, 9 \mathrm{D}$}

Hydrobiosella cerula Neboiss, 1977: 48.

\section{Holotype}

万ั Tasmania, Lake Pedder (original shores before flooding), 10 Mar. 1972 (NMV). 

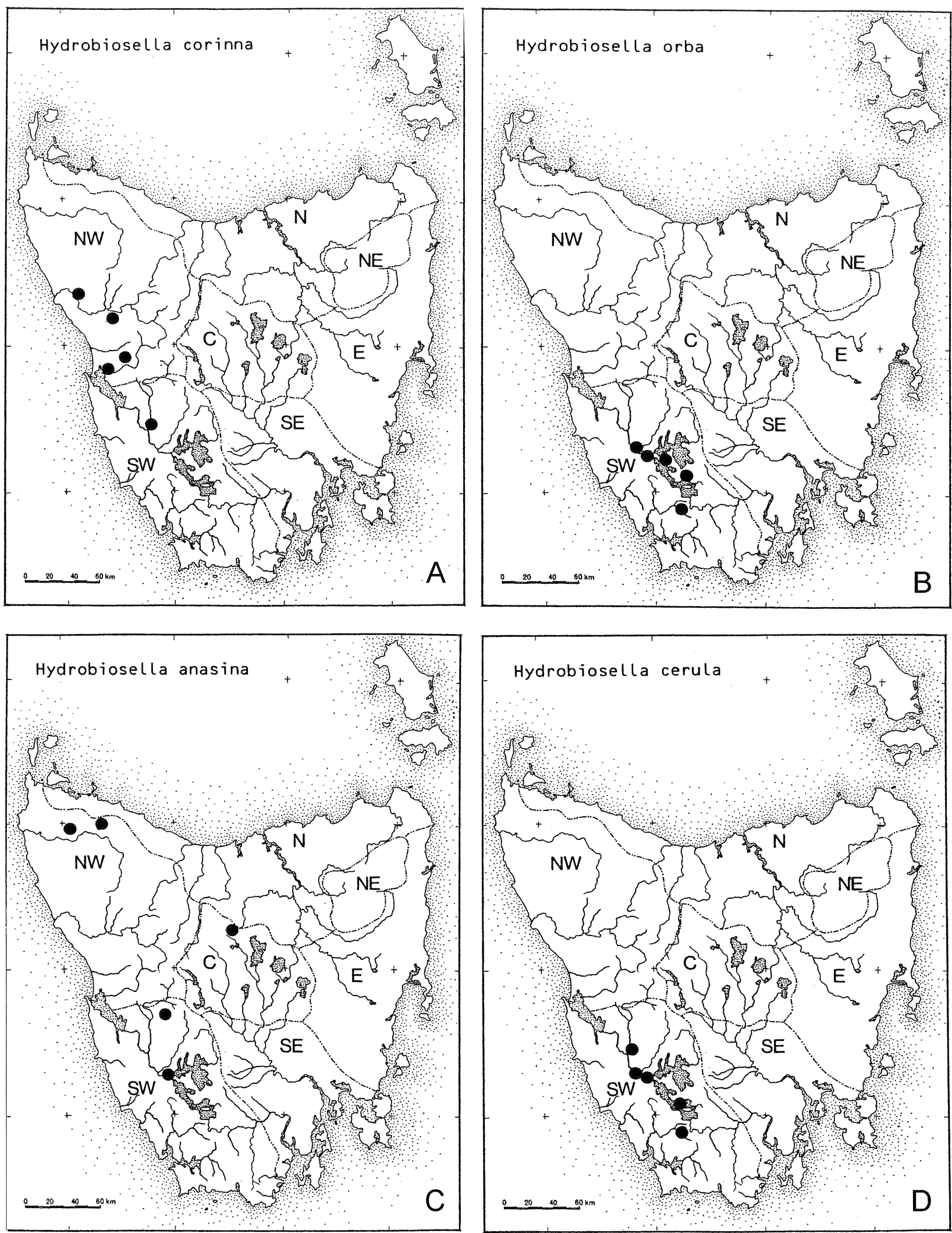

FIG. 9 - Distribution maps for Hydrobiosella spp.: (A) H. corinna; (B) H. orba; (C) H. anasina; (D) H. cerula. 


\section{Additional localities}

Tasmania, 40 Ropeway Creek, $400 \mathrm{~m}$ below Gordon and Smith River junction, 2 Feb. 1977, D.Coleman (male genitalia prep. PT-2262 illustrated); $30^{*}$ Franklin RiverRoaring Creek junction, $1 \mathrm{~km}$ above Gordon River, 8 Jan. 1977, A. Neboiss; $20^{*}$ Gordon River, 2 km below Serpentine River junction, 12 Jan. 1977, A. Neboiss; $10^{\star}$ Olga Camp near Gordon and Smith River junction, 2 Feb. 1976, Howard and Suter; 10 Lake Oberon, Western Arthur Ranges, top of plain, 9 Mar. 1999, J. Jackson. All specimens in NMV.

\section{Diagnosis}

Male genitalia (fig. $8 \mathrm{G}, \mathrm{H}$ ) similar to that in $H$. anasina, but differs by having the apex of segment $\mathrm{X}$ only gradually turned upwards; superior appendages present; inferior appendages long, harpago short, in lateral view sub-triangular, apex directed mesally and terminates bluntly with three or four strong claw-like spines. Phallus proportionally longer than in anasina, apex widened and covered with small irregularly dispersed spicules.

\section{Distribution}

Tasmania, SW province. Tasmanian endemic.

\section{tasmanica group}

This group appears to be a complex of a number of closely related species, which differs from each other only by details of segments IX and X in male genitalia. Based on present interpretation, ten species are recognised, each with rather restricted distribution as shown on the maps in figure 13AD. It should also be noted that all species of this group have a distinct curved bristle at the distal end of harpago.

\section{Hydrobiosella tasmanica Mosely Figs 10A, 10B, 13A}

Hydrobiosella tasmanica Mosely in Mosely \& Kimmins, 1953: 390; Neboiss 1977: 49.

\section{Holotype}

o Tasmania, (without definite locality or date) (BMNH).

In addition to the previously recorded localities further material has been collected which confirms the presence of this species in southern and southwestern Tasmania.

\section{Additional localities}

Tasmania. 20 Old River Collins River junction, 10 Feb. 1988, A. Neboiss (male genitalia prep. PT-2263 illustrated); $80^{\circ}$ d'Entrecasteaux River, South Cape road bridge, 14 Feb. 1988, A.Neboiss; $120^{*}$ Tomalah Creek, mid site, 9 Mar. 1988, S. Chilcott; $40^{*}$ Gordon River, $2 \mathrm{~km}$ below Serpentine River junction, 12 Jan. 1977, A. Neboiss; O (numerous) Condominion Creek, at Scotts Peak Road, 9 Feb. 1988, A. Neboiss and J. Jackson; lơ same loc., 26 Jan. 1988, J. Jackson; $30^{*}$ same loc. 2 Dec. 1998, J. Jackson; $10^{\star 1}$ Mulcahy Bay, Alec Rivulet, 12-15 Jan. 1988, J. Jackson. All specimens in NMV.

\section{Diagnosis}

Dark brown to brown species, forewings with irregular mottling. Male genitalia (fig. 10A, B) with distinctly shaped tergite IX, the lower angle of which is extended into long upcurved digital process. Segment X in dorsal view with small lateral flanges at about middle of the segment. Phallus slender, with a pair of strong, ventrally directed peg-like teeth, and a pair of small spines at distal end of dorsal membranous part.

\section{Distribution}

SE and SW provinces (fig. 13A). Tasmanian endemic.

\section{Hydrobiosella armata Jacquemart Figs 10C, 10D, 13B}

Hydrobiosella armata Jacquemart, 1965: 34; Neboiss 1977: 8C.

\section{Holotype}

O` Tasmania, Mt Wellington, 20 Nov. 1922 (IRScNB).

\section{Additional localities}

Tasmania. 20" Truggara Creek (Huon tributary) mid site, 9 Mar. 1988, S. Chilcott; $30^{\top}$ Tomalah Creek (Huon tributary) mid site, 9 Mar. 1988, S. Chilcott; $20^{*}$ d'Entrecasteaux passage, exit cave (1.7 km into dark zone), Jan. 1993, A. Clarke (male genitalia prep. PT-2048 illustrated).

\section{Diagnosis}

Although very close to $H$. tasmanica, $H$. armata is distinguished by the shorter and rounded tergite IX, and distinctly formed segment $\mathrm{X}$ as shown in figure 10C, D. Phallus without small terminal spines. $H$. armata is restricted mainly to the SE province, with only a few records from the SW province at Truggara and Tomalah Creeks where it occurs along with $H$. tasmanica. From these two localities there are several specimens that may be hybrids. The differences in the shape of tergite IX and segment $X$ are still regarded as sufficiently distinct to distinguish these two species.

\section{Distribution}

SE and SW provinces (fig. 13B). Tasmanian endemic.

\section{Hydrobiosella cognata Kimmins Figs 10G, 13C}

Hydrobiosella cognata Kimmins in Mosely \& Kimmins, 1953: 392; Jacquemart 1965: 32; Neboiss 1974: 15; Neboiss 1977: 50 .

Hydrobiosella spinosa Jacquemart 1965: 33.

\section{Holotype}

Ō Tasmania, Lake St Clair, Feb. 1941 (BMNH).

Distribution records as given by Neboiss (1977) with only one additional locality: $10^{\pi}$ Hellyer River Gorge, 12 Dec. 1974, A. Neboiss (male genitalia prep. PT-577 illustrated) NMV.

\section{Diagnosis}

Male genitalia (fig. 10G) tergite IX rounded dorsally, the digital upcurved process robust, apex more or less bluntly rounded; segment $\mathrm{X}$ in dorsal view only slightly wider at 


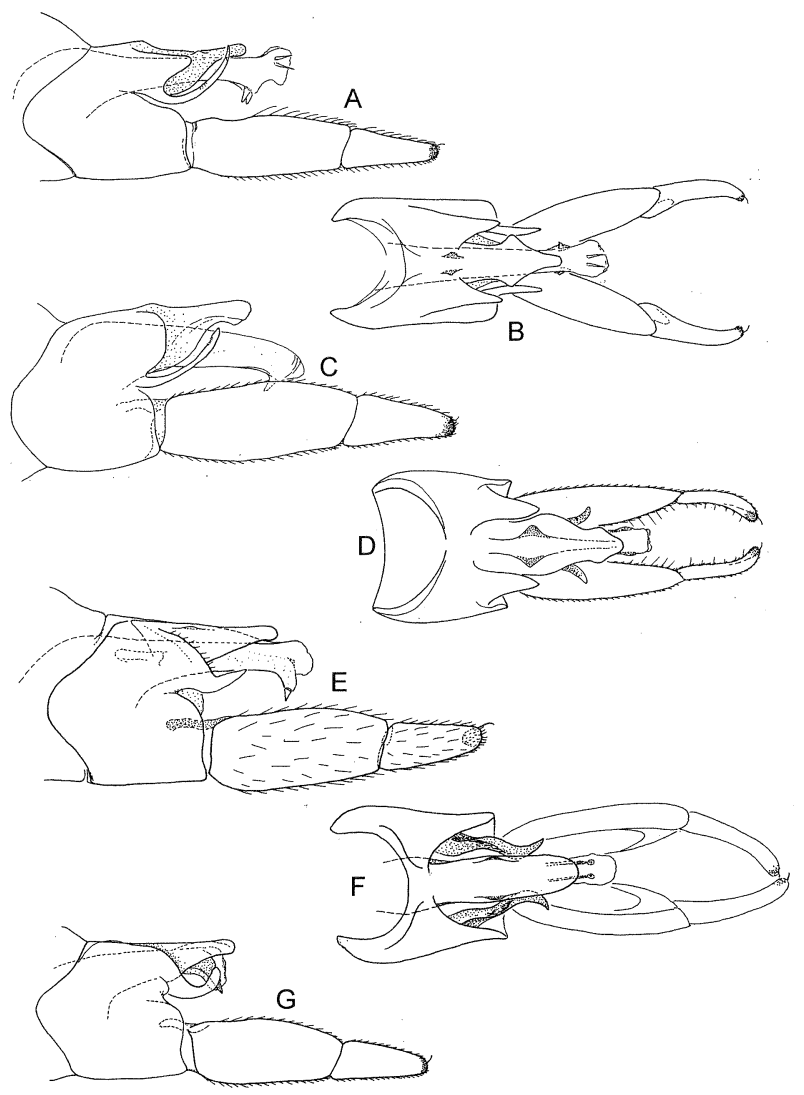

FIG. 10 - Hydrobiosella spp., male genitalia. $(A, B) \mathrm{H}$. tasmanica: (A) lateral; $(B)$ dorsal. $(C, D) \mathrm{H}$. armata: $(C)$ lateral; (D) dorsal; $(E, F) \mathrm{H}$. tahunense: $(E)$ lateral; $(F)$ dorsal. $(G) \mathrm{H}$. cognata, lateral.

base, with a pair of small triangular dorsal lobes. Phallus similar to that in $H$. tasmanica, but proportionally shorter; pair of downturned peg-like teeth present, the small terminal spines absent.

Distribution

N, C and NW provinces (fig. 13C). Tasmanian endemic.

\section{Hydrobiosella tahunense sp. $\mathrm{n}$.} Figs $10 \mathrm{E}, 10 \mathrm{~F}, 13 \mathrm{C}$

\section{Type material}

Holotype ${ }^{\top}$ Tasmania, Lake Tahune, Frenchmans Cap area, 29 Jan. 1988, J. Jackson; Paratypes $30^{\circ}$ collected with holotype (male genitalia prep. PT-1702 illustrated); $10^{*}$ Nelson River below Lyell Highway bridge, 3 Nov. 1988, J. Jackson; $10^{\top}$ Cardigan River above Lyell Highway bridge, 16 Feb. 1999, J. Jackson. All specimens in NMV.

\section{Diagnosis}

Greyish-brown to brown species; irregular dark spots along costal margin. Length of forewing o 6.6-7.3 mm (Nelson River specimen $8.3 \mathrm{~mm}$ ). Male genitalia (fig. 10E, F) similar to that of $H$. tasmanica but tergite IX dorsal margin gradually curved, lower distal angle produced into pointed, slightly upcurved digital process; segment X almost parallel-sided, widest at basal third with small triangular lobes; phallus straight with pair of strong downward directed peg-like teeth; upper membranous section without internal spines.

\section{Etymology}

Named after the type locality — Lake Tahune.

\section{Distribution}

NE and SW provinces (fig. 13C). Tasmanian endemic.

\section{Hydrobiosella anatolica sp. $\mathrm{n}$. Figs 11A-11C, 13B}

\section{Type material}

Holotype $0^{\prime}$ Tasmania, Fortescue Bay, Mill Creek $43^{\circ} 08^{\prime} \mathrm{S}$, $147^{\circ} 58^{\prime} \mathrm{E}, 28$ Feb. 2001, R. Walker. Paratypes $40^{\circ} 1$ \% collected with holotype (male genitalia preps PT-2251, female PT-2252 illustrated); $10^{\pi}$ Anglers Creek, Little Swanport, 13 Oct. 1977, D. Coleman. All specimens in NMV.

\section{Diagnosis}

Greyish-brown species, forewings with irregular mottling along costal margin. Length of forewing $07.2-7.7 \mathrm{~mm}$; $ᄋ$ $7.9 \mathrm{~mm}$. Male genitalia (fig. 11A, B) with division between tergite and sternite of segment IX short; distal part of tergite squarish, lower distal angle with slightly upcurved digital process; segment $X$ in dorsal view with lateral lobes near apex and a pair of triangular lobes near base which are species specific. Phallus with strong downward directed peg-like teeth, membranous part without terminal spines. Female (fig. 11C) with one pair of apodemes in segments VIII and IX.

\section{Etymology}

Anatole (Gr.) - east, in reference to the distribution of the species.

\section{Distribution}

$\mathrm{E}$ and SE provinces - Tasmanian east coast (fig. 13B). Tasmanian endemic.

\section{Hydrobiosella sagitta Neboiss Figs 11D, 11E, 13B}

Hydrobiosella sagitta Neboiss, 1977: 51.

\section{Holotype}

ర大 Tasmania, St Columba Falls nr Pyengana, 21 Feb. 1971 (NMV).

Only one additional specimen has been collected since the species was described in 1977 and it also came from the vicinity of the type locality. $10^{\pi} \mathrm{St}$ Columba Falls, Pyengana, 10 Mar. 1982, D. Cartwright (NMV).

\section{Diagnosis}

Distictively formed tergite IX, segment $\mathrm{X}$ and phallus distinguishes it from all other species in Tasmania.

\section{Distribution}

NE province, known only from type locality (fig. 13B). Tasmanian endemic. 


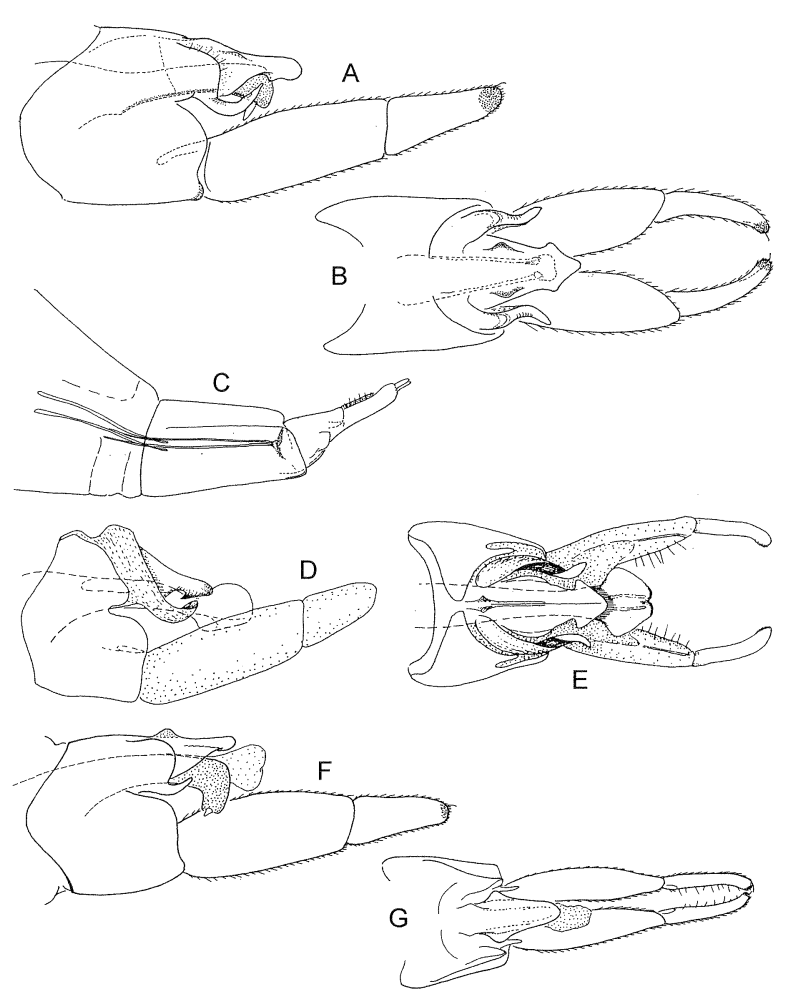

FIG. 11 - Hydrobiosella spp. $(A-C) \mathrm{H}$. anatolica: (A) male genitalia lateral; $(B)$ male genitalia dorsal; $(C)$ female genitalia, lateral. $(D, E) \mathrm{H}$. sagitta male genitalia: $(D)$ lateral; $(E)$ dorsal. $(F, G) \mathrm{H}$. disrupta male genitalia: $(F)$ lateral; $(G)$ dorsal.

\section{Hydrobiosella disrupta sp. $\mathrm{n}$. Figs 11F, 11G, 13D}

\section{Type material}

Holotype O Tasmania, Gordon River, $500 \mathrm{~m}$ below Second Split, 12 Jan. 1977, A. Neboiss. Paratypes $40^{\circ}$ collected with holotype (malegenitalia prep. PT-575 illustrated); $10^{\pi}$ Gordon River, $1 \mathrm{~km}$ above First Split, 11 Jan. 1977, A. Neboiss (NMV).

\section{Diagnosis}

Yellowish-brown species with slight mottling on forewings. Length of forewing - $0^{\pi} 7.7-8.3 \mathrm{~mm}$. In basic form, the male genitalia (fig. $11 \mathrm{~F}, \mathrm{G}$ ) resemble those of $H$. tasmanica, but are distinguished by having tergite IX shorter and more rounded, ventro-apical digital process rather short, slightly upcurved; segment X short, similar to that of $H$. propinqua, in dorsal view widest near base formed by elevated triangular lateral flanges. Phallus with pair of peg-like teeth directed downward at apex.

\section{Etymology}

Disruptus (L) - broken up, in reference to type of terrain at the type locality.

\section{Distribution}

SW province - known only from the type locality (fig. 13D). Tasmanian endemic.
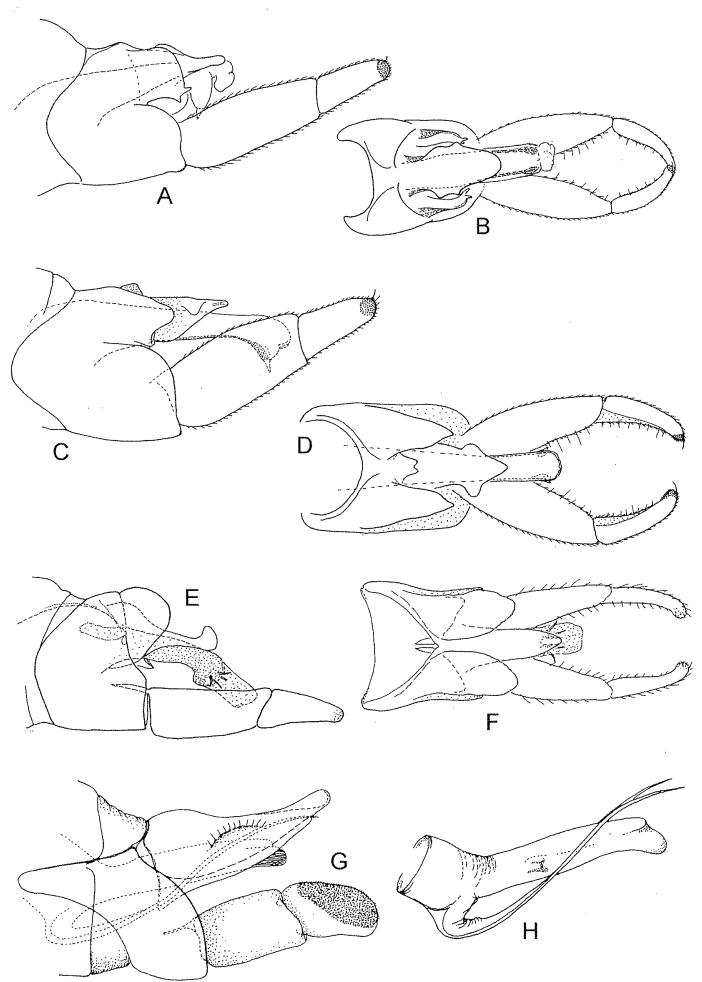

FIG. 12 - Hydrobiosella spp., male genitalia. $(A, B) \mathrm{H}$. propinqua: $(A)$ lateral; $(B)$ dorsal. $(C, D) \mathrm{H}$. scalaris: $(C)$ lateral; (D) dorsal. $(E, F)$ H. otaria: (E) lateral; $(F)$ dorsal. $(G, H)$ H. waddama: $(G)$ lateral; $(H)$ phallic structure.

Hydrobiosella propinqua sp. $\mathrm{n}$. Figs 12A, 12B, 13D

Type material

Holotype ${ }^{\star}$ Tasmania, Smith River at Gordon River junction, 15 Jan. 1977, A. Neboiss. Paratypes $30^{\star}$ collected with holotype; $10^{\top}$ Smith River, $100 \mathrm{~m}$ from Gordon River junction, 7 Feb. 1977, D. Coleman (male genitalia prep. PT-2193 illustrated); 80 Franklin River, Roaring Creek Junction, 1 km above Gordon River, 8 Jan. 1977, A.Neboiss. All specimens in NMV.

\section{Other material examined}

Tasmania, 30’ Sir John Falls, Cataract Creek, Gordon River tributary, 9 Jan. 1977, A. Neboiss, NMV.

\section{Diagnosis}

Yellowish-brown to light brown species, sometimes with mottling on forewings. Length of forewing $0^{\pi} 7.8-8.6 \mathrm{~mm}$. Similar to $H$. tasmanica, but distinguished by the squarish, distally produced tergite IX and two small digitiform processes at the apico-ventral angle; segment $\mathrm{X}$ rather short, in dorsal view it has two pairs of lateral flanges (fig. 12A).

\section{Etymology}

Prope (L) - near, in reference to the similarity of other Hydrobiosella species.

\section{Distribution}

SW province (fig. 13D). Tasmanian endemic. 

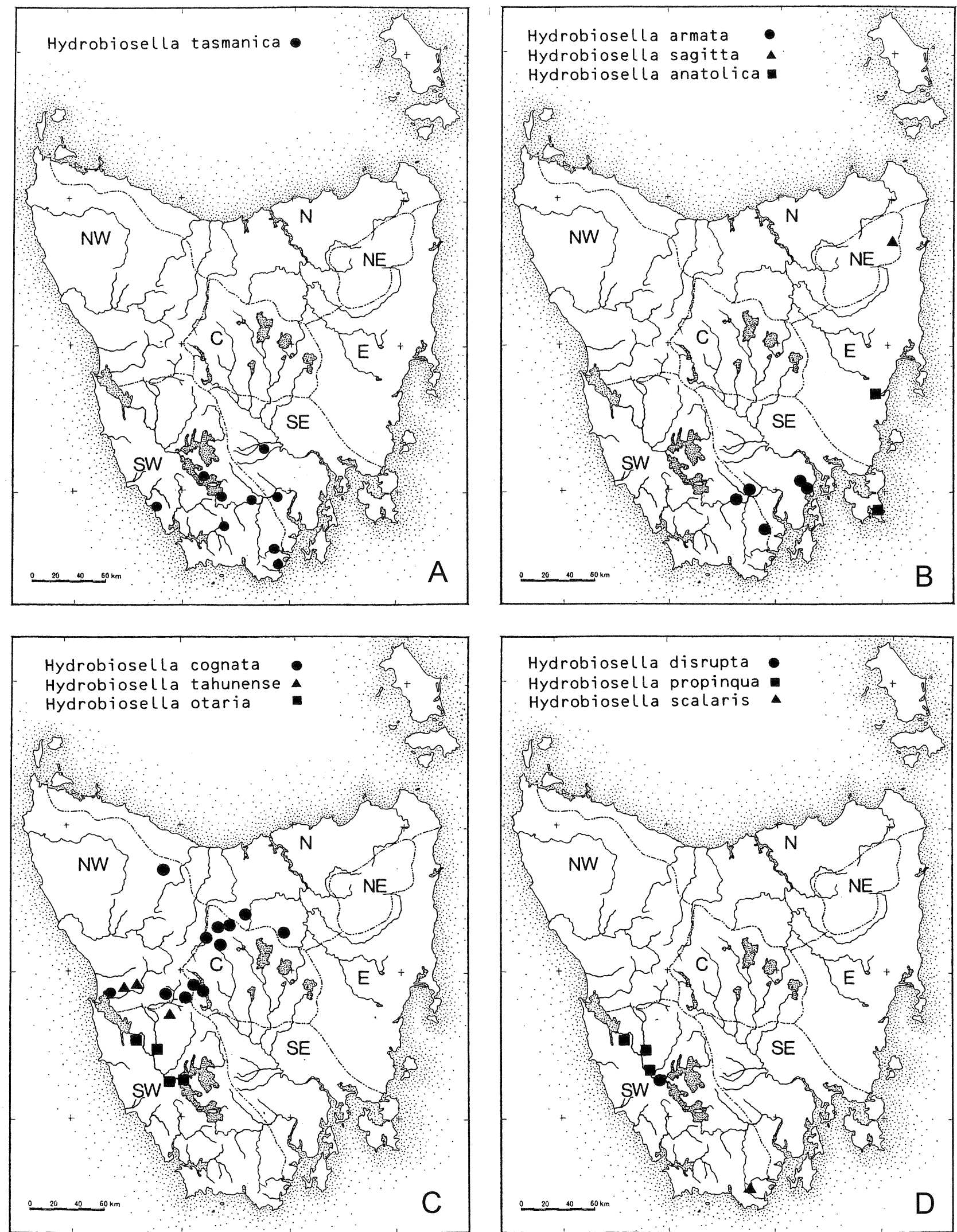

FIG. 13 - Distribution maps for Hydrobiosella spp.: (A) H. tasmanica; (B) H. armata, H. anatolica and H. sagitta; (C) H. cognata, H. tahunense and H. otaria; (D) H. disrupta, H. propinqua and $\mathrm{H}$. scalaris. 


\section{Hydrobiosella scalaris sp. $\mathrm{n}$. Figs 12C, 12D, 13D}

\section{Type material}

Holotype $0^{\star}$ Tasmania, d'Entrecasteaux River source, below Mt LaPerouse, $43^{\circ} 30^{\prime} \mathrm{S}, 146^{\circ} 44^{\prime} \mathrm{E}$, alt. $840 \mathrm{~m}$ (sweep net), 16 Feb. 1988, A. Neboiss (male genitalia prep. PT-2202 illustrated), NMV.

\section{Diagnosis}

Pale yellowish-brown species, without obvious mottling. Length of forewing $0^{*} 8.2 \mathrm{~mm}$. Male genitalia of similar plan to that in $H$. tasmanica, but distinguished by the total absence of apico-ventral process on tergite IX, upper margin developed into a rounded lobe; segment $X$ with a pair of somewhat rounded lateral lobes near distal end and an elevated dorsal bipointed projection at base. Phallus with pair of stout peg-like teeth at apex.

\section{Etymology}

Scalaris (L) - of a ladder, in reference to the elevation of the type locality.

\section{Distribution}

SW province, known only from the type locality (fig. 13D). Tasmanian endemic.

\section{Hydrobiosella otaria sp. $\mathrm{n}$. Figs $12 \mathrm{E}, 12 \mathrm{~F}, 13 \mathrm{C}$}

\section{Type material}

Holotype $0^{\pi}$ Tasmania, Sir John Falls, Cataract Creek, Gordon River tributary, 42³4'S, 145²1'E, 9 Jan. 1977 A. Neboiss. Paratypes $80^{*}$ collected with holotype (male genitalia prep. PT-567 illustrated); $50^{\star}$ Gordon River, $500 \mathrm{~m}$ below Second Split, 12 Jan. 1977, A. Neboiss; 30 Gordon River, $1 \mathrm{~km}$ above First Split, 11 Jan, 1977, A. Neboiss; $20^{\star}$ Franklin River-Roaring Creek junction, $1 \mathrm{~km}$ above Gordon River, 8 Jan. 1977, A. Neboiss; $110^{\star}$ Strathgordon, Jan. 1977, J. Sedlacek. All specimens in NMV.

\section{Diagnosis}

Greyish-brown to brown species, forewing with indistinct mottling. Length of forewing $06.3-6.9 \mathrm{~mm}$. Male genitalia (fig. 12E, F) segment IX short, tergite distally developed into large ear-like lobe, lower mesal angle somewhat pointed; segment $\mathrm{X}$ in dorsal view elongate triangular, apex raised to a short vertical ridge. Phallus slender, slightly curved downward, one pair of $V$-shaped chitinous spines near apex.

\section{Etymology}

Otos $(\mathrm{Gr})$ - ear, in reference to the shape of tergite IX.

Distribution

SW province (fig. 13C). Tasmanian endemic.

\section{waddama group}

\section{Hydrobiosella waddama Mosely}

Fig. 12G, H

Hydrobiosella waddama Mosely in Mosely \& Kimmins, 1953: 393; Neboiss 1977: 52.

\section{Holotope}

OT Tasmania, Waddamana, River Ouse (no date) $(\mathrm{BMNH})$.

This well-known and common species is widely distributed throughout most of Tasmania, but there are no records from the E province. This is the only species of the group in Tasmania. All others are in Victoria, New South Wales and Queensland. The main distinguishing characteristics are the more robust inferior appendages, rounded harpago with a large patch of short, black setae and phallus with a pair of long processes arising from its base (fig. $12 \mathrm{H})$.

\section{Additional localities}

Tasmania - $10^{\top}$ Denison River, 200 m below Maxwell River junction, 10 Jan. 1977, A. Neboiss; $10^{\star} 1$ Q Port Arthur, Jan. 1977, J. Sedlacek; $10^{\star}$ Gordon River, $200 \mathrm{~m}$ above Olga River junction, (no date), D. Coleman; 90" Franklin River above Lyell Highway bridge, 16 Feb. 1999, J. Jackson; 1o Scotts Peak Dam, Serpentine impoundment, 23 Mar. 1985, P. Greenslade; $60^{\star}$ Agnes Creek, Fortescue Bay, 28 Feb. 2001, R. Walker; $0^{\top}$ o (numerous 50+) Pinetree Rivulet, Lake Highway near Breona, 9. Feb. 2001, R. Walker (male genitalia prep. PT-2258 illustrated); $10^{\circ} 1$ 우 Doctors Creek at Lake Highway nr Breona, 9 Feb. 2001, R. Walker. All specimens in NMV.

\section{Distribution}

Tasmania, widespread, common. Other states: Victoria, New South Wales.

\section{FAMILY ECNOMIDAE}

The family Ecnomidae in Tasmania is represented by three genera - Ecnomus McLachlan, Ecnomina Kimmins and Daternomina, a new genus introduced in this paper. This genus has been erected to include some species previously placed in Ecnomina, in which the hind wing anal veins are distinctly bent and the female sternite VIII is divided into two lobes. Two new species are also described in this genus.

\section{Genus Ecnomus McLachlan}

Ecnomus McLachlan, 1864: 30; Neboiss 1977: 54; Cartwright 1990: 21.

\section{Type species}

Philopotamus tenellus Rambur, 1842 by monotypy.

The genus in Tasmania is represented by four species E. tillyardi Mosely, E. russellius Neboiss, E. turgidus Neboiss and E. cygnitus Neboiss. This latter name replaces $E$. continentalis Ulmer. The identification of this species was based on illustration in Mosely \& Kimmins (1953: fig. 261), which was prepared from a South Australian specimen, and as such was wrongly identified. The true identity of $E$. continentalis was established when the lectotype was reexamined and figured by Neboiss (1982). To allow positive recognition of the Tasmanian species the figure of $E$. continentalis is included (fig. 14G). 


\section{Ecnomus cygnitus Neboiss Figs $14 \mathrm{~A}-\mathrm{C}, 15 \mathrm{C}, 15 \mathrm{D}, 18 \mathrm{~A}$}

Ecnomus cygnitus Neboiss, 1982: 288; Cartwright 1990: 16.

Holotype

O Victoria, Swan Lake, $30 \mathrm{~km}$ NW of Portland, 27 Feb. 1976 (NMV).

\section{Tasmanian records}

$50^{7} 9$ o West Bay River, Margate, 6 Jan. 1977, A. Neboiss and P. Allbrook (male genitalia prep. PT-1069 illustrated); $10^{\star}$ Carlton River, Carlton, 26 Dec. 1975, M. Davies; $10^{7}$ South Esk River, Evandale, 1 Mar. 1967, A. Neboiss (recorded as E. continentalis by Neboiss 1977); $10^{\star}$ Apsley River, $5 \mathrm{~km}$ NW of Bicheno, 20 Dec. 1988, J. Jackson; lo" Lake Pedder, Site 'J' Dec. 1977, P.S. Lake; 60' 3o Lake Fiddler, Lower Gordon River, 13 Dec. 1977, D. Coleman; lo Scotts Peak Dam, Serpentine impoundment, 23 Apr. 1985, Greenslade and Rounsevell; 10 ' 1 o Arve River, 3 Feb. 1983, J. Cardale; $10^{\star} 2$ Q Lake Pedder, shore N of Forest Creek, 12 Feb. 1999 , J. Jackson;170 5\% Maria Creek near Lake Pedder, 10 Feb. 1999, J. Jackson; $10^{\star}$ Pebbly Creek, 50 m upstream of Lake Pedder, 10 Feb. 1999, J. Jackson; 20" stream South of

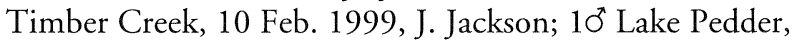
Frankland shore, 10 Feb. 1999, J. Jackson; $10^{\star}$ Doctors Creek at Lake Highway near Breona, 9 Feb. 2001, R. Walker. All specimens in NMV.

Male genitalia (fig. 14A-C) with superior appendages in lateral view robust, broadest in median section; inferior appendages in ventral view with subapical mesal projection at about the middle, separated widely from apical angle.

Female genitalia (fig. 15C, D) with rounded pocket near meso-basal angle; ventral plate slightly concave.

\section{Distribution}

Tasmania, scattered locality records from E, C and SW provinces. Other states: Victoria, South Australia, New South Wales, Queensland.

\section{Ecnomus tillyardi Mosely Figs 14D, 14E, 15E, 15F}

Ecnomus tillyardi Mosely in Mosely \& Kimmins, 1953: 378; Neboiss 1977: 55; Cartwright 1990: 14.

\section{Holotype}

ऽ Tasmania (without definite locality or date) (BMNH).

\section{Distribution}

This is the most common and widespread of Ecnomus species in Tasmania. Large numbers are collected throughout the Southwest National Park with collecting dates ranging from December through to March. Other states: South Australia, Victoria, New South Wales.

\section{Ecnomus turgidus Neboiss Fig. 14F}

Ecnomus trugidus Neboiss, 1982: 289; Cartwright 1990: 8; Jackson 2000: 59.

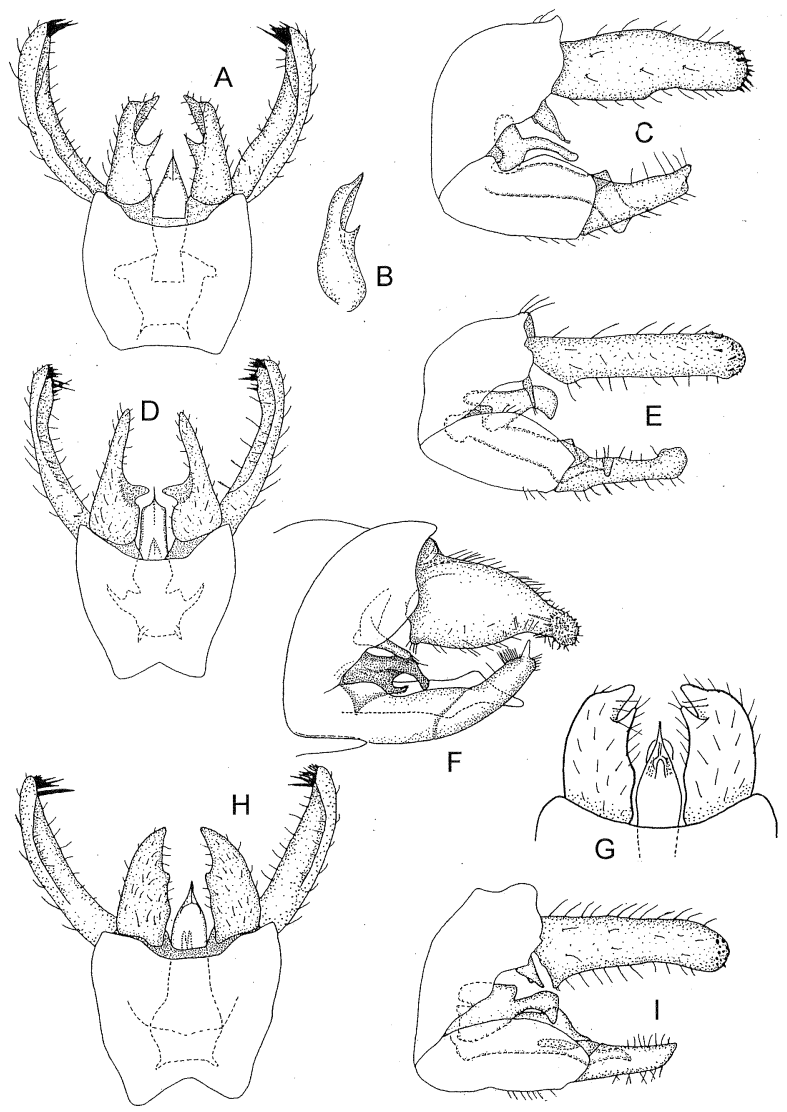

FIG. 14 - Ecnomus spp., male genitalia. (A-C) E. cygnitus: (A) ventral; (B) inferior appendage - variety; $(C)$ lateral. (D, E) E. tillyardi: (D) ventral; $(E)$ lateral. $(F)$ E. turgidus: lateral (Tasmanian specimen CT-274). (G) E. continentalis, ventral. $(H, I)$ E. russellius: $(H)$ ventral; (I) lateral.

\section{Tasmanian records}

$10^{\star}$ Maria Creek nr Lake Pedder, 10 Feb. 1999, J. Jackson and B. Mawbey (NMV) (male genitalia prep. CT-274 by Cartwright illustrated).

Male genitalia (fig. 14F) with superior appendages in lateral view broad-based, tapering distally, slightly constricted before rounded apex which bears a group of inflected spines on inner surface; a rounded lobe present on ventral margin near base; inferior appendages in lateral view slender, apically with distinct, dorsally directed digital projection; paramere in lateral view differs from the typical form by second, ventrally situated lobe. At present this character state is not regarded as sufficient for species separation, and more material is required to clarify the variability of the paramere.

\section{Distribution}

Tasmania - SW province. Other states: Western Australia, South Australia, Victoria, Queensland.

\section{Ecnomus russellius Neboiss Figs $14 \mathrm{H}-15 \mathrm{~B}$}

Ecnomus russellius Neboiss, 1977: 56; Cartwright 1990: 22. 

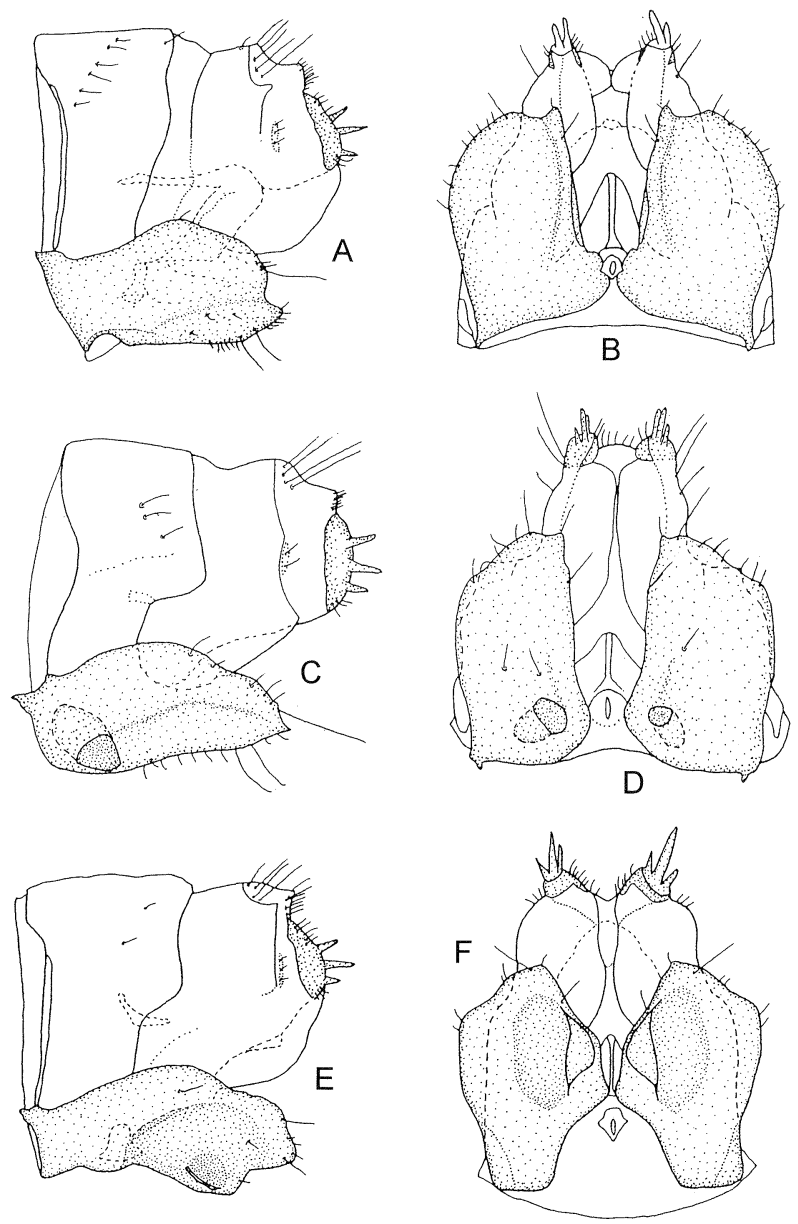

FIG. 15 - Ecnomus spp., female genitalia. (A, B) E. russellius: $(A)$ lateral; $(B)$ ventral. $(C, D)$ E. cygnitus: $(C)$ lateral; (D) ventral. (E, F) E. tillyardi: (E) lateral; (F) ventral.

\section{Holotype}

ơTasmania, Russell Falls, 20 Feb. 1967 (NMV).

In addition to the localities recorded since in the Ecnomus revision by Cartwright (1990), 30', Doctors Creek at Lake Highway near Breona, 9 Feb. 2001, R. Walker (NMV).

\section{Distribution}

Tasmania, C and E provinces. Other states: Victoria, New South Wales, SE Queensland.

\section{Genus Ecnomina Kimmins}

Ecnomina Kimmins in Mosely \& Kimmins, 1953: 380; Neboiss 1977: 57.

\section{Type species}

Ecnomina spinosa Kimmins by original designation.

The genus Ecnomina s.str. is characterised by absence of nygma in forewing fork 2; comparatively long fork 3, either reaching or ending close to cross-vein $m$ at a distance not exceeding the length of cross-vein. Hind wing comparatively narrow, anal veins running almost straight from base of the wing to margin. In female the abdominal sternite VIII extends into more or less narrow, elongate mesal process. The larvae of 'sentosa' group from SW Australia as identified by Neboiss (1982), to which three Tasmanian species also belong, are considered as typical of the genus Ecnomina and described by Dean \& Bunn (1989). It is characterised by subovate head capsule; medium length, robust ligula; all thoracic nota sclerotised; mesonotum and metanotum with median ecdysial line, and also each with narrow dark line extending obliquely back from near anterolateral corner.

Three Tasmanian species - batyle Neboiss, legula Neboiss, and vega Neboiss - are referred to this genus.

\section{Ecnomina legula Neboiss Fig. $16 \mathrm{~A}, \mathrm{~B}$}

Ecnomina legula Neboiss 1977: 58.

\section{Holotype}

○ Tasmania, Huon River Crossing, Port Davey Track, 9 Feb. 1965 (NMV).

\section{Additional Tasmanian material examined}

Numerous $O^{\star}$ and $O$ Maria Creek near Lake Pedder, 10 Feb. 1999, J. Jackson; 4<0' 60 d'Entrecasteaux River at South Cape road bridge, 14 Feb. 1988, A. Neboiss and K. Walker; $200^{\pi}$ o Mulcahy Bay area, Alec Rivulet tributary, 12-15 Jan. 1988, J. Jackson. All specimens in NMV.

\section{Distribution}

Tasmania, scattered localities, common where present. Tasmanian endemic.

\section{Ecnomina batyle Neboiss}

Ecnomina batyle Neboiss, 1977: 59.

Holotype

ơTasmania, Wilmot River, $10 \mathrm{~km} \mathrm{~S} \mathrm{of} \mathrm{Forth,} 13$ Dec. 1974 (NMV).

\section{Additional localities}

50 5o West Bay River, Margate, 6 Jan. 1977, A. Neboiss (NMV).

\section{Distribution}

Tasmania, N, C and SE provinces, scattered localities only, rare. Tasmanian endemic.

\section{Ecnomina vega Neboiss}

Ecnomina vega Neboiss, 1977: 58.

Holotype

đ' Tasmania, Macquarie River, 8 km W of Campbell Town, 9 Nov. 1972 (NMV).

\section{Distribution}

Tasmania E province, known from type locality only. No additional specimens available. Tasmanian endemic. 


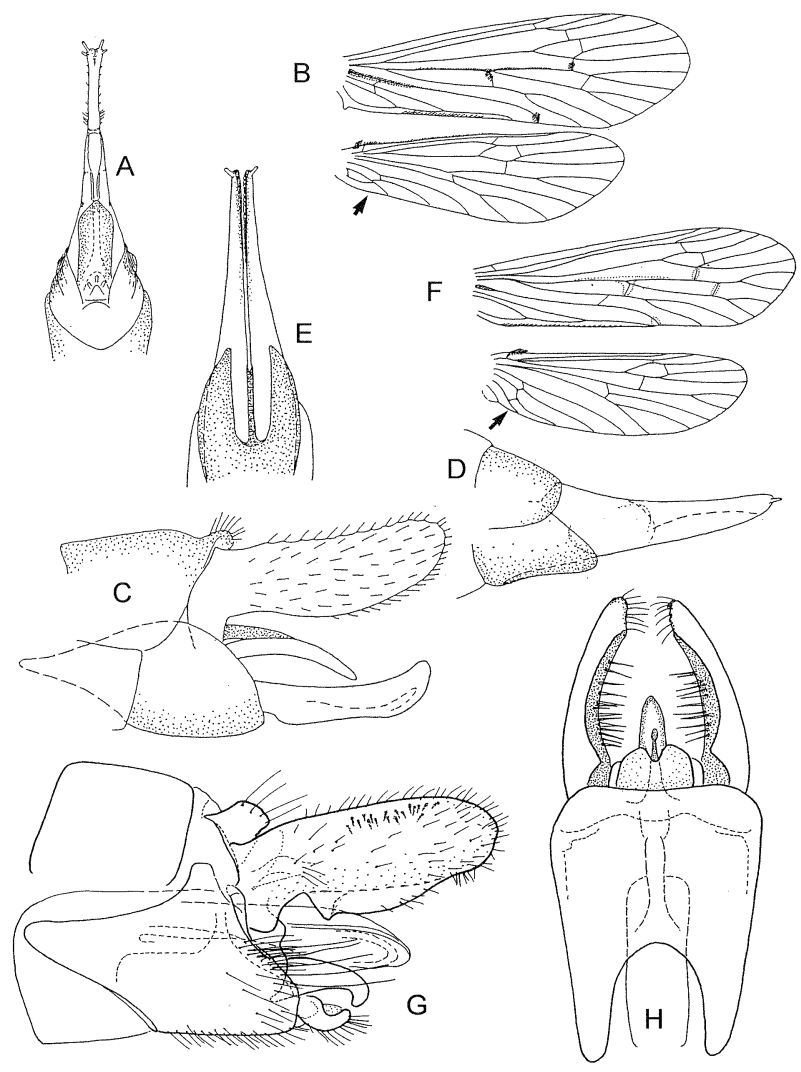

FIG. $16-(A, B)$ Ecnomina legula Neboiss: (A) female genitalia ventral; $(B)$ male wing venation. $(C-F)$ Daternomina irrorata (Kimmins): (C) male genitalia lateral; (D) female genitalia lateral; (E) female genitalia ventral; $(F)$ female, wing venation. $(G, H)$ Daternomina quasitrulla $s p$. n., male genitalia: $(F)$ lateral; $(H)$ ventral.

\section{Genus Daternomina gen. nov.}

\section{Type species}

Ecnomina irrorata Kimmins, 1953, by present designation.

Neboiss (1982) recognised two distinct species groups within the genus Ecnomina s.lat. when describing the SW Australian fauna. At that time it was also realised that this division is applicable to all Ecnomina species in Australia. Support for this grouping from larval characters was discussed by Cartwright \& Dean (1982) and by Dean \& Bunn (1989).

The 'sentosa' group, to which the type species Ecnomina spinosa Kimmins from New South Wales belongs, is characterised by forewing fork 2 without nygma, comparatively long fork 3 with very short footstalk, about as long or shorter than cross vein $\mathrm{m}$; rather narrow hind wings with anal veins almost straight. The female abdomen with sternite VIII developed into an elongate meso-ventral lobe (fig. 16A). This group represents Ecnomina s.str. The 'scindens' group, including Ecnomina irrorata Kimmins, is diagnosed by forewing fork 2 with nygma present, fork 3 with footstalk longer than cross-vein $\mathrm{m}$; hind wing somewhat broader with distinctly curved anal veins. The female abdomen with sternite VIII formed by lateral lobes widely separated meso-ventrally (fig. 16E). This group is here removed to a new genus, Daternomina.

\section{Description}

Maxillary palps slender, segment 5 about as long as the first four together; forewing R1 forked; fork 1 absent; fork 2 sessile, nygma present; fork 3 comparatively short, footstalk slightly longer than the cross-vein $\mathrm{m}$; hind wing forks 2,3 and 5 present; anal veins distinctly curved at the level of connecting cross veins. Male abdominal sternite $\mathrm{V}$ anterolaterally with a small ear-shaped lobe, connected internally with a small round gland (as described by Ward \& Schefter (2000).

Larvae recognised as the 'scindens' group were characterised by Dean \& Bunn (1989) as follows: head capsule broadest near posterior margin. Ligula long and needle-like. All thoracic nota sclerotised. Meso- and metanotum with sclerotisations reduced, resulting in broad membranous area along midline. Dark lines on mesonotum branched, situated more dorsally than in species of the 'sentosa' group [= Ecnomina s. str.].

This genus includes $D$. irrorata, and the southwestern Australian species scindens (Neboiss), trulla (Neboiss) and merga (Neboiss); the SE Queensland krokale (Neboiss), chorisis (Neboiss) and thinotes (Neboiss); and two species newly described here, quasitrulla and jacksonae from Tasmania.

\section{Etymology}

Daterios - Gr. dividing, combined with part of generic name Ecnomina, in reference to the deeply divided sternite VIII in females.

\section{Daternomina irrorata (Kimmins) comb. nov. Fig. 16C-E}

Ecnomina irrorata Kimmins in Mosely \& Kimmins, 1953: 385; Neboiss 1977: 57.

\section{Holotype}

ơ Tasmania, Deloraine, 26-27 Dec. 1884, McLachlan Coll. (BMNH).

\section{Additional localities}

Tasmania - O o Mulcahy Bay area, Alec Rivulet tributary, 12-15 Jan. 1988, J. Jackson; $10^{\star}$ Twin Creeks, Scotts Peak Dam road, 9 Feb. 1988, J. Jackson; $10^{\pi}$ Old River and Collins River junction 10 Feb. 1988, J. Jackson; 1 \% Sandfly Creek, Scotts Peak road, 9. Feb. 1988, K. Walker and J. Jackson; 1 Condominion Creek, Scotts Peak Dam road, 9 Feb. 1988, A. Neboiss and K. Walker; 20 d'Entrecasteaux River, South Coast road, 14 Feb. 1988, A. Neboiss and K. Walker; $10^{\star} 2$ O Apsley River, $5 \mathrm{~km}, \mathrm{NW}$ of Bicheno, $20 \mathrm{Dec}$. 1988, J. Jackson; đo o Lake Pedder, shore N of Forest Creek, 10 Feb. 1999, J. Jackson; Ơ $\$$ Maria Creek near Lake Pedder, 10 Feb. 1999, J. Jackson; $10^{\pi}$ Pebbly Creek, about 50 m upstream of Lake Pedder, 10 Feb. 1999, J. Jackson; 307 70 Lake Pedder, Frankland shore, 10 Feb. 1999, J. Jackson. All specimens in NMV.

The species was described fully by Kimmins in Mosely \& Kimmins (1953) and further details and distributional data were added by Neboiss (1977). Wing venation (fig. $16 \mathrm{~F}$ ), male (fig. 16C) and female (fig. 16D, E) genitalia are illustrated here for ease of comparison. 


\section{Distribution}

Tasmania, widespread throughout the state. Tasmanian endemic.

\section{Daternomina quasitrulla sp. $\mathrm{n}$. Figs $16 \mathrm{G}, 16 \mathrm{H}, 18 \mathrm{~B}$}

\section{Type material}

Holotype $0^{\prime}$ Tasmania, Fortescue Bay $43^{\circ} 08^{\prime} \mathrm{S}, 147^{\circ} 57^{\prime} \mathrm{E}$, Tasman Penninsula [wet sclerophyl forest, collected in Mailaise trap], 7-9 Feb. 1989, D. Bickle (male genitalia prep. PT-1834 illustrated), NMV.

Length of forewing: $0^{\pi} 4.9 \mathrm{~mm}$. Adults small, brownish, closely resembling the SW Australian species Daternomina trulla (Neboiss 1982) from the Albany area, but may be distinguished by details of male genitalia, particularly the robust mesal process at the base of inferior appendages.

Male genitalia (fig. 16G, H): abdominal sternite IX elongate, distal margin with a row of long bristles, which exceed the length of inferior appendages; tergite IX membranous, separated from the short membranous segment $\mathrm{X}$ by a strong ridge; superior appendages large, rather robust with elongate group of spines on upper mesal margin; inferior appendages fused, forming a short ventral plate, cleft apically; a robust mesal process, apically bent downward and slightly exceeding the length of inferior appendages.

Female unknown.

\section{Etymology}

Quasi (L) - simulating, like, in reference to the similarity to the SW Australian species D. trulla.

\section{Distribution}

SE province; known from type locality only. Tasmanian endemic.

\section{Daternomina jacksonae sp. $\mathrm{n}$. Figs $17 \mathrm{~A}-\mathrm{E}, 18 \mathrm{~B}$}

\section{Type material}

Holotype $0^{\top}$ Tasmania, Lake Pedder, Frankland shore, $42^{\circ} 58^{\prime} \mathrm{S}, 146^{\circ} 09^{\prime} \mathrm{E} ; 10$ Feb. 1999 , J. Jackson. Paratypes $70^{\circ}$ 2 o collected with holotype (male genitalia preps PT-2249 and female PT-2189 illustrated); $20^{\circ}$ Lake Pedder impoundment WHA, 1 Mar. 1988, SC/JJ; $10^{\top}$ Lake Pedder, Frankland shore, 2 Mar. 1988,J. Jackson; lo Lake Pedder, Teds Beach S corner, 4 Mar. 1988, J. Jackson; 10 Lake Pedder, Kostka Point, Cumberland shore, 2 Mar. 1988, J. Jackson; $10^{\star} 1$ Stream E of Spring St. houses, Strathgordon, 13 Jan. 1999, J. Jackson. All specimens in NMV.

\section{Other material examined}

$10 * 10$ O Olga River, $19 \mathrm{~km}$ above Gordon River junction, 13 Jan. 1977, A. Neboiss; 1 + Swamp near Olga River, 19 km above Gordon River junction, 13 Jan. 1977, A. Neboiss; $10^{7}$ Louisa Creek, 25 Dec. 1978, S.F. McEvey; lo Apsley River, $5 \mathrm{~km} \mathrm{NW}$ of Bicheno, 20 Dec. 1988, J. Jackson (NMV).

Adults brown to dark brown; length of forewing $0^{*} 4.3$ $4.6 \mathrm{~mm}$; $9.6-5.0 \mathrm{~mm}$. This species closely resembles the SW Australian species $D$. scindens, but differs in genitalic details.

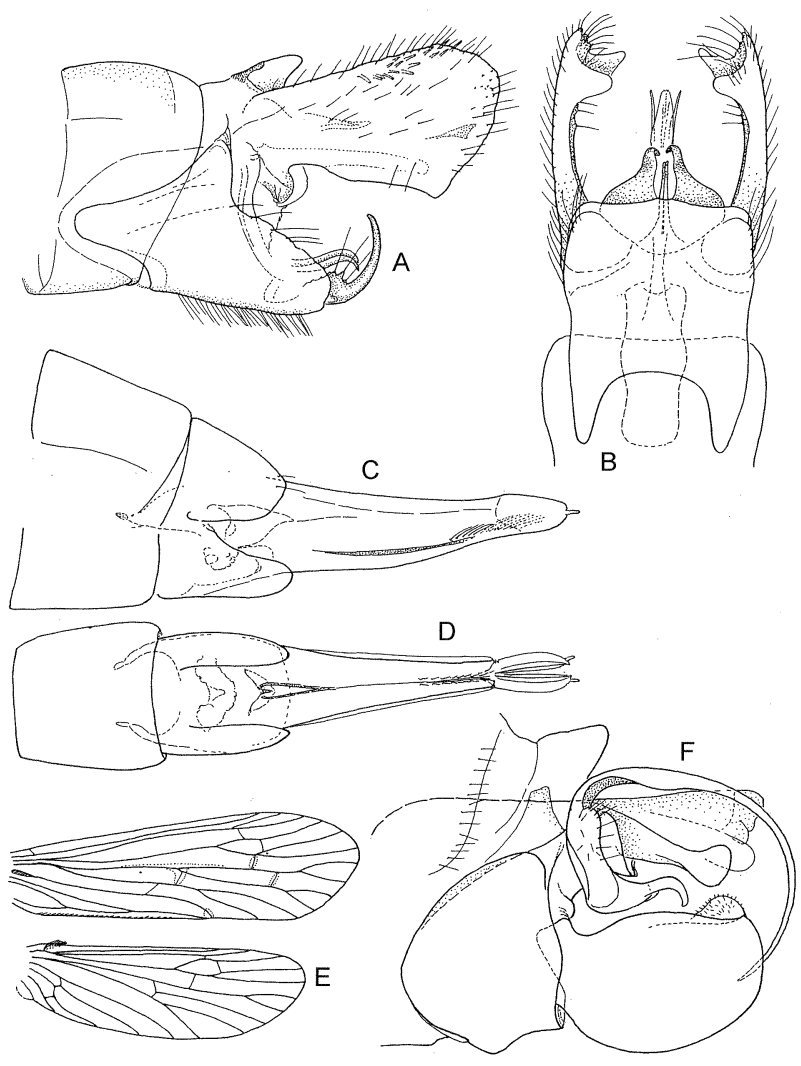

FIG. 17-Daternomina.jacksonae sp. n.: (A) male genitalia lateral; (B) male genitalia ventral; (C) female genitalia lateral; (D) female genitalia ventral; $(E)$ male wing venation. $(F)$ Polyplectropus lacusalbinae Kimmins, male genitalia lateral.

Male genitalia (fig. 17A, B) segment IX membranous dorsally, separated from the membranous segment $\mathrm{X}$ by a distinct ridge; superior appendages large, widened distally, lower margin with mesally directed distal lobe, inner surface of dorsal margin with elongate group of spines and a triangular mesal lobe near distal end. Inferior appendages upturned, claw-shaped; a pair of close, slender, curved mesal processes arising from the base; phallus straight, membranous.

Female abdomen (fig. 17C, D) slender, gradually tapered distally; sternite VIII widely divided meso-ventrally, forming two lateral plates, sternite IX elongate slender, with darkly pigmented, distally pointed, mesal margins at base.

\section{Etymology}

Named after Dr Jean Jackson for her contribution to knowledge of Tasmanian caddis flies.

\section{Distribution}

Tasmania, SW province. Tasmanian endemic.

\section{FAMILY POLYCENTROPODIDAE}

\section{Polyplectropus UImer}

Polyplectropus Ulmer, 1905: 103; Mosely \& Kimmins 1953: 359. 


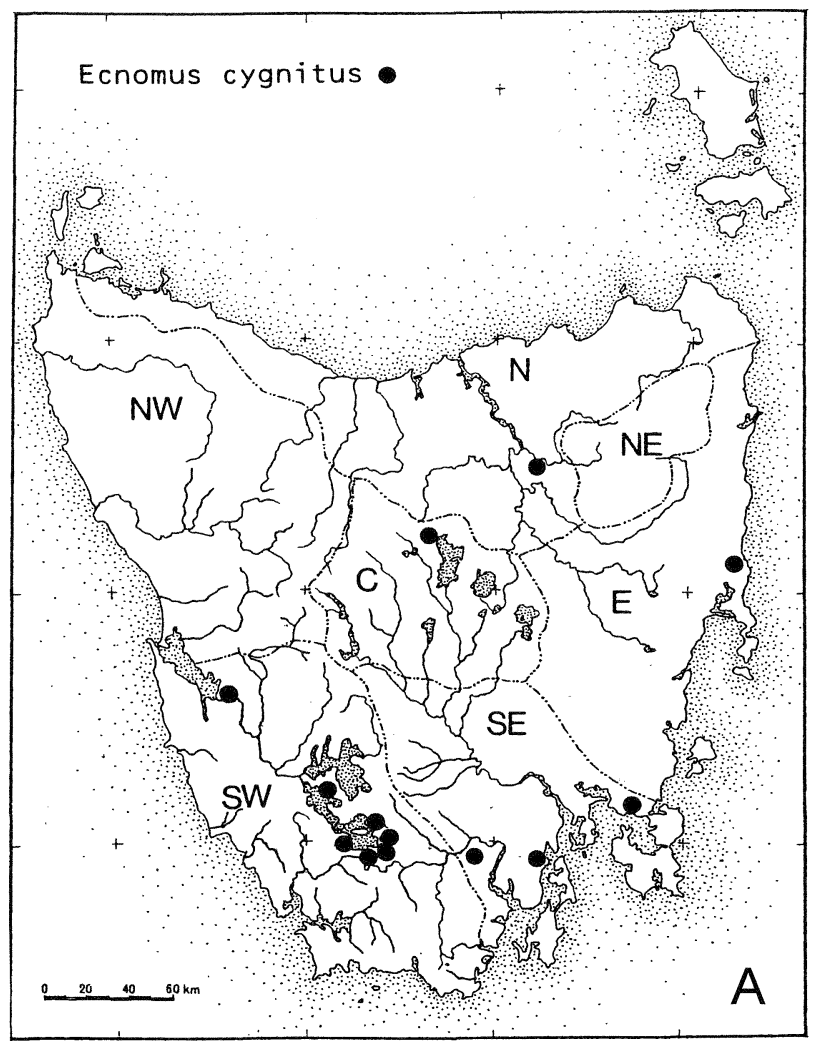

FIG. 18 - Distribution maps: (A) Ecnomus cygnitus Neboiss;

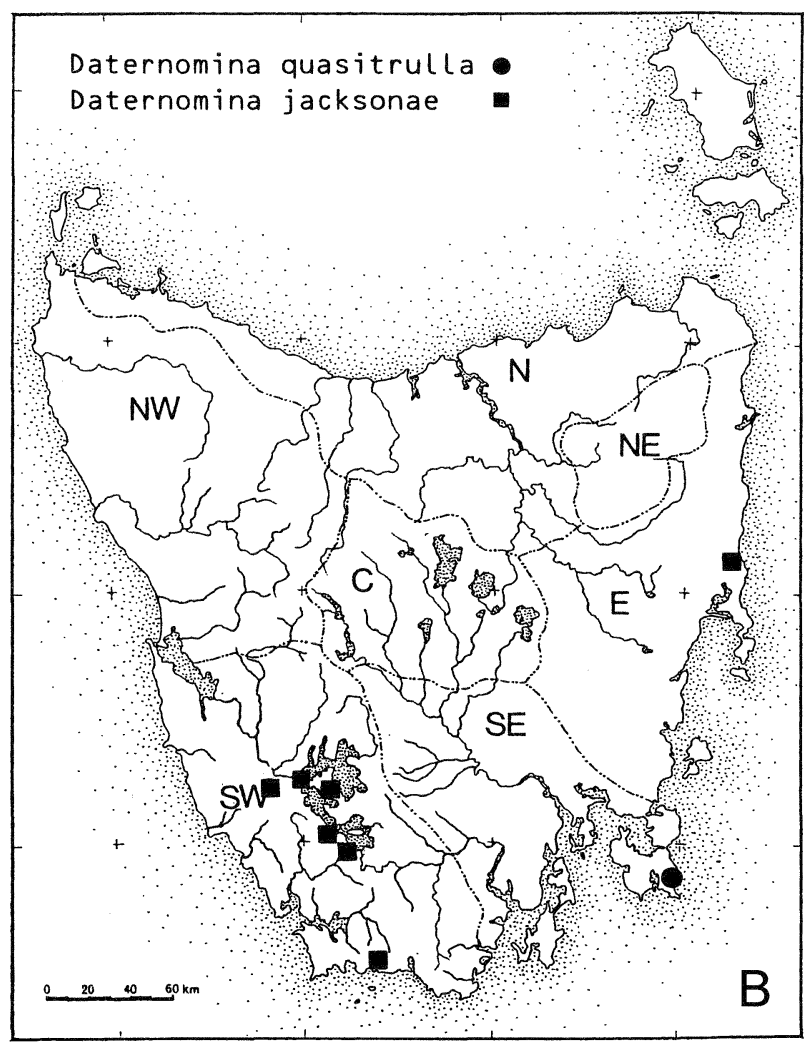

(B) Daternomina quasitrulla $s p . n$. and D. jacksonae $s p . n$.
Type species

Polyplectropus flavicornis Ulmer, 1905 by monotypy.

\section{Polyplectropus lacusalbinae Kimmins} Fig. 17F

Polyplectropus lacusalbinae Kimmins in Mosely \& Kimmins, 1953: 363, fig. 249.

\section{Material examined}

Tasmania $10^{\star}$ Flinders Island, Pats River area, 3 Feb. 1963, N. Dobrotworsky (male genitalia prep. PT-1694 illustrated). This species is here recorded for the first time from Victoria — IO Noojee, Loch Valley, 9 Apr. 1959, N. Dobrotworsky. All specimens in NMV.

Originally described from a single 0 from Lake Albina, Mt Kosciusko, New South Wales, this species is now recorded more widely for first time. It resembles somewhat the New Zealand species Polyplectropus puerilis (McLachlan). Other species of the genus are known from South-West Pacific localities, and it would not be surprising if $P$. lacusalbinae is more widespread in Tasmania.

\section{Diagnosis}

Dark, greyish-brown species, forewing $0^{\star} 6.3-8.0 \mathrm{~mm}$ with numerous golden irrorations; fork 1 present; hind wing smoky greyish brown with forks 2 and 5 only. Male genitalia (fig. 17F) segment IX dorsally membranous, fused with segment X; sternite IX strongly chitinised. Dorsal to inferior appendages is a complex sclerite, dorsal margin of which forms a long downwards curved strap-like spine; the second branch is in form of an elongate rounded lobe directed outward; a third, lower branch forms a down and outwardly directed hook; interior appendages single-segmented, robust with rounded dorso-mesal hairy lobe. Phallus cylindrical, lower margin developed into an elongate lateral flange with margin upcurved.

Female unknown.

\section{Distribution}

Flinders Island. Other states: Victoria, New South Wales.

\section{Genus Paranyctiophylax Tsuda}

Paranyctiophylax Tsuda, 1942: 265; Neboiss 1993: 110.

\section{Type species}

Paranyctiophylax kisoensis Tsuda, 1942 by original designation.

The generic concept of the genus Nyctiophylax Brauer was reviewed by Neboiss (1993). The genus Paranyctiophylax Tsuda was redefined to accommodate a distinct lineage of species previously included in Nyctiophylax. In this analysis 25 species were assigned to the genus Paranyctiophylax, among them also the only Tasmanian species, $P$. repandus (Neboiss, 1977).

The genus Paranyctiophylax is diagnosed as having forewing fork 1 absent, fork 3 present and anal veins distinctly looped and thickened near the junction; in the hind wing forks 1 and 3 absent, discoidal cell present and cell at the base of A2-A3 long; cross-vein closing cell distinctly separated from cross-vein $\mathrm{Cu} 2-\mathrm{A} 1$ and closer to the wing margin. 


\section{Paranyctiophylax repandus (Neboiss)} Fig. 19A

Nyctiophylax repandus Neboiss, 1977: 65.

Paranyctiophyla repandus. Neboiss 1993: 107.

Holotype

O Tasmania, Scamander River, Upper Scamander, 9 Nov. 1972 (NMV).

\section{Additional material examined}

Tasmania 10 Sir John Falls, Cataract Creek, Gordon River tributary, 9 Jan. 1977, A. Neboiss et al. $50^{\star} 2$ o Franklin River Roaring Creek junction, $1 \mathrm{~km}$ above Gordon River, 8 Jan. 1977, A. Neboiss et al.; 30 1\% West Bay River, Margate, 6 Jan. 1977, A. Neboiss and P. Allbrook; 70 lo Apsley River, $5 \mathrm{~km} \mathrm{NW}$ of Bicheno, 20 Dec. 1988, J. Jackson. All specimens NMV.

\section{Distribution}

Tasmania, scattered localities NE, E, SE and SW provinces. Tasmanian endemic.

\section{Genus Tasmanoplegas Neboiss}

Tasmanoplegas Neboiss, 1977: 64.

Type species

Tasmanoplegasspilota Neboiss, 1977 by original designation.

\section{Tasmanoplegas spilota Neboiss}

Tasmanoplegas spilota Neboiss, 1977: 64.

\section{Holotype}

ð Tasmania, West Arthur Plains, 26 Feb. 1965 (NMV).

\section{Material examined}

Tasmania 150 Strathgordon, Jan. 1977, J. Sedlacek; $10^{\star}$ Dove River below Dove Lake, 18 Jan. 1976, A. Wells; $30^{\circ}$ Franklin River, buttongrass plain, 14 Jan. 1983, ANZSES expedition; $20^{*}$ Swamp near Olga River, $12 \mathrm{~km}$ above Gordon River junction, 12 Jan. 1977, A. Neboiss; $20^{\circ}$ small trickle flowing into Lake Ayr, Cradle Mountain-Lake St Clair National Park, 16 Jan. 1990, J. Dean and D. Cartwright; 50 Lake St Clair, 21 Dec. 1999, M. Driessen. All specimens in NMV.

The additional localities are all associated with small streams on buttongrass plains. The species appears to be rather widely distributed and locally common. The preparation PT-256 of the original and only female is somewhat damaged and thus comparison with the specimens from the south coast is very difficult.

\section{Distribution}

Tasmania, scattered localities NW and SW provinces. Tasmanian endemic.

\section{Tasmanoplegas sp.} Fig. 19B-D

\section{Material examined}

Tasmania 2Q Cavers' camp, NE New River Lagoon, 31 Dec. 1978, S.E. McEvey (genitalia prep. PT-1712 illustrated).

Length of forewing: $O^{*}$ unknown; $99.7 \mathrm{~mm}$.

The wing pattern (fig. 19B) of these south coast specimens appears to be very similar to that of T. spilota. However, the only known female of Tasamanoplegas spilota is distinctly smaller (forewing $8 \mathrm{~mm}$ ). The female genitalia, although very similar, also show some differences, but with the absence of males from the south coast locality, the species identity remains unresolved.

\section{FAMILY HYDROPSYCHIDAE}

\section{Genus Cheumatopsyche Wallengren}

Cheumatopsyche Wallengren, 1891: 142; Neboiss 1977: 66.

Type species

Hydropsyche lepida Pictet, 1834 (Europe) by monotypy.

Moderate-sized, slender insects; wings usually greyishbrown to yellowish-brown, more or less distinctly mottled. Cross-veins $\mathrm{M}_{3+4}-\mathrm{Cu} 1$, and $\mathrm{Cu} 1-\mathrm{Cu} 2$ in forewing close to each other. Hind wing fork 1 absent.

The genus is widely distributed in Australia, but until recently only one species, Cheumatopsyche modica (McLachlan), was recognised. Detailed studies of larvae by Dean (1999) reveal a far greater diversity of species. Previously all specimens from Tasmania were ascribed to Cheumatosyche modica (Neboiss 1977), but the existence was noted of two distinct forms, one marginally paler and larger, the other slightly smaller and darker. Closer examination of genitalic characters revealed subtle but constant differences in character states, enabling their separation into two distinct species. This division is supported by the distinct larval types as recognised by Dean (1999). An ovoid cellular structure/patch (fig. 20I) is located on pleurite of segment IV. This structure is present in females of both Tasmanian species as well as in the females of $C$. modica from the type locality - Merri Creek, Victoria. This may be regarded as a generic character.

In Tasmania Cheumatopsyche alampeta sp. n. appears to be more widespread than Cheumatopsyche deani sp. n. It also appears to be more common in mid to late summer (December-January) and is more often associated with smaller streams than is $C$. deani sp. n. A single male from Lake Tahune, Frenchmans Cap area, 28 Jan. 1988 (Coll. J. Jackson; PT-2164) may represent another undescribed species, but more material is required to verify its identity.

When the drawing of Cheumatopsyche modica type became available (courtesy of P. Barnard, NMH, pers. comm.) it was apparent that none of the Tasmanian specimens conforms with $C$. modica in all character states, particularly in details of segments IX and X, as well as inferior appendages in males and the shape of scleritic cavity in females (fig. 20C, G). Thus new names are established for the Tasmanian species and at this stage, the name $C$. modica is excluded from the Tasmanian fauna and replaced with $C$. deani sp. n. and C. alampeta sp. n. 

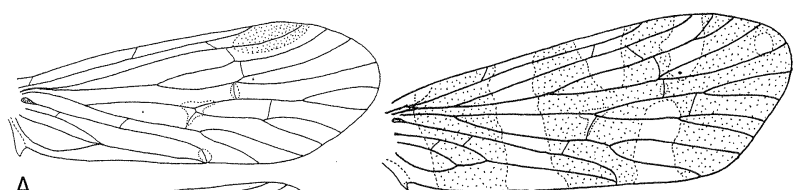

A
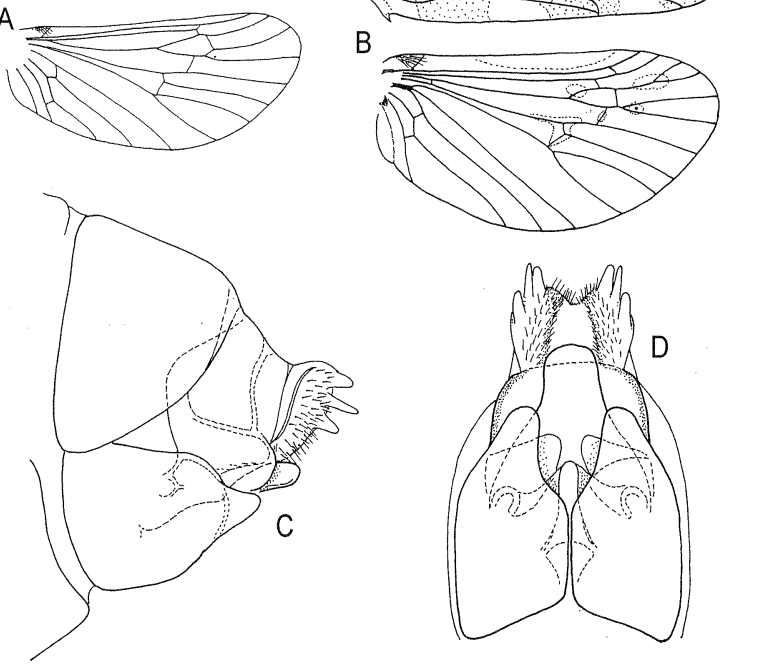

FIG. 19 - (A) Paranyctiophylax repandus (Neboiss), wing venation. ( $B-D)$ Tasmanoplegus sp. female: (B) wing venation; (C) female genitalia lateral; (D) female genitalia ventral.

\section{Cheumatopsyche deani sp. $\mathrm{n}$. Fig. 20A-D}

\section{Type material}

Holotype Ođ, Tasmania, Leven River, Heka, 17 Nov. 1972, A. Neboiss (male genitalia prep. PT-2235 illustrated). Paratypes $150^{\top} 15$ o same data as for holotype (female genitalia prep. PT-2234 illustrated); 30"9 9 Mersey River, Liena, 16 Nov. 1972, A. Neboiss. All specimens in NMV.

\section{Other material examined}

Tasmania, $10^{*} 860$ Scamander River, Upper Scamander, 9 Nov. 1972, A, Neboiss; 20 South Esk River, Evandale, 1 Mar. 1967, A. Neboiss. All specimens in NMV.

\section{Description}

Forewings greyish-brown to yellowish-brown, indistinctly mottled. Length of forewing: $0^{\pi} 9.8-10.4 \mathrm{~mm}$; o 10.1-11.0 $\mathrm{mm}$.

Male genitalia (fig. 20A, B) segment IX in lateral view with basal margin rather uniformly curved, the height:length proportions usually 2.1:1; dorsal segment $\mathrm{X}$ broad, subquadrate with broad mesal excision, apico-lateral lobes short, rounded; pre-anal appendages squat, rounded; inferior appendages slender, harpago about $1 / 4$ to $1 / 5$ length of coxite; distal end of coxite gradually and only slightly widened.

Female genitalia (fig. 20C, D) sternite VIII somewhat rounded, margins not obviously, if at all, pigmented; scleritic cavity (Nielsen 1980), also known as clasper receptacle, with distal margin usually extending further ventrad than basal margin, opening about full width of the cavity; dorsal separation of cavities usually is slightly less than one width of each cavity.

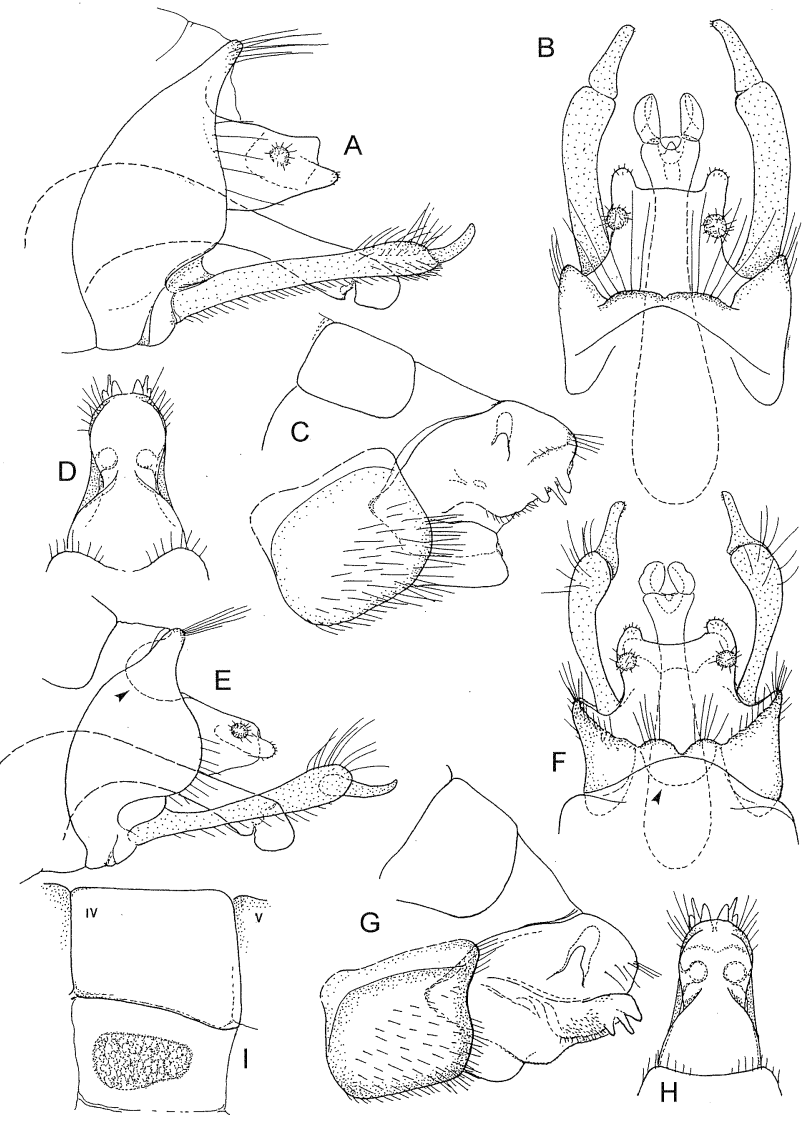

FIG. $20-(A-D)$ Cheumatopsyche deani sp. n. A, B male genitalia: $(A)$ lateral; $(B)$ dorsal. $(C, D)$ female genitalia: $(C)$ lateral; (D) dorsal. (E-I) Cheumatopsyche alampeta $s p$. $n$. $(E, F)$ male genitalia: $(E)$ lateral; $(F)$ dorsal. $(G, H)$ female genitalia: $(G)$ lateral; $(H)$ dorsal. (I) tergite and pleurite IV lateral.

\section{Distribution}

In Tasmania, usually found in slow flowing lowland streams. Other states: probably Victoria and New South Wales.

\section{Cheumatopsyche alampeta sp. $\mathrm{n}$. Fig. 20E-I}

\section{Type material}

Holotype $0^{7}$ Tasmania, Derwent River, $2 \mathrm{~km} \mathrm{NW}$ of Derwent Bridge, 12 Feb. 1971, A. Neboiss, NMV. Paratypes 150 15 same data as for holotype. (Male genitalia prep. PT2232 and female PT-2231 illustrated.)

\section{Other material examined}

Tasmania, 107 Derwent River, $3 \mathrm{~km} \mathrm{~W}$ of New Norfolk, 7 Dec. 1974; $90^{\circ} 8$ o Plenty River, $6 \mathrm{~km}$ E of Moogara, 7 Dec. 1974, A. Neboiss; 220 20 West Bay River, Margate, 6 Jan. 1977, A. Neboiss; 70 10 Shannon River, Wihareja, 29 Nov. 1982, W. Fulton; $10^{*} 1$ Q Shannon River, 15 Dec. 1982, W. Thompson; $20^{\star} 2 Q$ Liawanne, laboratory aquarium, Dec. 1982, W. Fulton; $110^{\star} 1$ o Black Bobs Creek, $15 \mathrm{~km} \mathrm{~N}$ of Ouse, 9 Dec. 1974, A. Neboiss; 40 19ᄋ Ouse River, 8 km W of Miena, 14 Dec. 1982, W. Fulton; $10^{*} 1$ p Bronte Park, 12 km NNE, 20 Jan. 1983, J. Cardale; $30^{\star} 160$ Hellyer River Gorge, 9 Feb. 1971, A. Neboiss; 440 480 Mersey River, 
Liena, 16 Nov. 1972, A. Neboiss; 10 1o Leven River, Heka, 17 Nov. 1972, A. Neboiss; 280 140, Macquarie River, 8 km W of Campbelltown, 9 Nov. 1972, A. Neboiss; $30^{\pi} 2$ o Lake River, 5 km SW Delmont, 9 Nov. 1972, A. Neboiss; $20^{\circ}$ Tooms Lake, 4 Dec. 1974, A. Neboiss; $20^{\star} 1$ 1 Apsley River, $5 \mathrm{~km}$ NW of Bicheno, 20 Dec. 1988, J. Jackson; $10^{\pi}$ Styx River, Westerway, 23 Nov. 1972, A. Neboiss; 110 1\% Scamander River, Upper Scamander, 9 Nov. 1972, A. Neboiss; $10^{*}$ Lilydale, small creek, 2 km N, 16 Dec. 1974, A. Neboiss. All specimens in NMV.

\section{Description}

Adults with general colour of forewings dark blackishbrown with slight mottling. Length of forewing: $0^{\pi} 7.9-8.4$ $\mathrm{mm}$; $7.8-9.0 \mathrm{~mm}$. Male genitalia (fig. 20E, F) segment IX in lateral view with basal margin depressed dorsally; the height:length proportions approx. 1.9:1; lateral lobe more pronounced than in deani; segment $\mathrm{X}$ broad, mesal excision broad; apical apico-lateral lobes short, rounded; pre-anal appendages squat, rounded; the base of tergite $\mathrm{X}$ forms an obvious rounded cavity within the dorsal section of segment IX clearly visible in cleared preparations; inferior appendages slender, harpago about $1 / 4$ length of coxite; distal end of coxite widened, almost club-like and usually with darker pigmentation.

Female genitalia (fig. 20G, H), sternite VIII somewhat angular with apico-dorsal angle more or less produced; all margins more or less strongly pigmented; scleritic cavity with both margins of approximately the same length, opening less than full width of the cavity, distal margin with an obvious bend about midway; dorsal separation of cavities about half to one width of the cavity.

\section{Etymology}

Alampetos (Cr.) - dark, obscure in reference to its generally darker colour.

\section{Distribution}

Tasmania, widespread. Other states: probably Victoria.

\section{Genus Diplectrona Westwood}

Diplectrona Westwood, 1840: 49; Neboiss 1977: 71.

\section{Type species}

Aphelocheira flavomaculata Stephens, 1836 by original designation.

The species listed from Tasmania by Neboiss (1977) are here revised. It is now confirmed (Neboiss 1986) that Diplectrona bispinosa Jacquemart belongs to the genus Austropsyche Banks, and the type locality is indeed Sassafras, Victoria. Thus, this species is removed from the Tasmanian list.

Additional material collected during the last 20 years has led to further clarification of this group. The re-examination of the type of Diplectrona castanea Kimmins reveals that the original figures published by Mosely \& Kimmins (1953: fig. $237 \mathrm{~b}-\mathrm{d}$ ) fail to show all the details critical for species identification: particularly, these authors omit the shape of lateral margin of segment IX, and some details of the phallic structures. Examination of these features indicated that Diplectrona lyelli Neboiss, 1977 is synonymous with D. castanea.

Species of the genus Diplectrona s.l. in Australia may be divided into several more or less distinct groups, two of which are represented in Tasmania. One species $D$. castanea belongs to the Diplectrona s.l., but two others, D. tasmanica Jacquemart and a new species, are referred to a new genus, Diemeniluma.

The genera Diplectrona and Diemeniluma in Tasmania may be distinguished by the following characteristics.

Diplectrona: head with five setal warts (fig. 21A). Male genitalia with lateral margin of segment IX extended into a distinct triangular lobe; phallic apparatus with parameres. Female genitalia with sternite VIII consisting of two sclerites separated ventro-mesally.

Diemeniluma: Head with four setal warts (fig. 22A), antero-mesal wart absent. Male genitalia with lateral margin of segment IX almost straight, mot extended into a lobe; phallic apparatus simple, base bulbous, parameres absent. Female genitalia with sternite VIII consisting of a single sclerite.

\section{Diplectrona castanea Kimmins Fig. 21A-D}

Diplectrona castanea Kimmins in Mosely \& Kimmins, 1953: 342; Neboiss 1977: 72.

Diplectrona lyelli Neboiss, 1977: 72. New synonym.

Holotype of Diplectrona castanea, O Tasmania, National park, 3000 ft, 26 Dec. 1936 (BMNH); (male genitalia illustrated).

Holotype of Diplectrona lyelli, O Tasmania, King River, 5 Nov. 1961 (NMV).

\section{Additional material examined}

Tasmania, $10^{\star}$ Hellyer River Gorge, 2 Dec. 1972, P. Zwick (paratype of D. lyella; aedeagus prep. PT-1043 illustrated); $10^{\star} 10$ Nelson Falls, 3 Nov. 1998, J. Jackson; $10^{\star}$ Little Florentine River upstream of Gordon road, 2 Dec. 1998, J. Jackson; 1o Bull Creek, Cradle Mountain road, 12 Dec. 1974, A. Neboiss; $10^{\star}$ tributary of Darwin River, West Coast, 31 Oct. 1976, P. Albrook; 19 Arrowsmith Creek, 18 km SW of Derwent Bridge, 9 Dec. 1974, A. Neboiss (female genitalia prep. PT-1068 illustrated); all specimens in NMV. $10^{7}$ Mt Burrow, $11 \mathrm{~km}$ EbyN Nunamara, 7 Feb. 1983, J. Cardale (ANIC).

\section{Diagnosis}

Dark blackish-brown in colour. Length of forewing 078.4 $10.0 \mathrm{~mm}$; $10.2-12 \mathrm{~mm}$. Male abdominal segments VI and VII each with a pair of globular internal sacks (glands); genitalia (fig. 21B); segment IX lateral margin with distinct dorsally pointed lobe; inferior appendages stout, coxite slightly widened at apex, about 2.5 times length of harpago. Phallus (fig. 21C), with two pairs of parameres, the inner pair slender, almost as long as aedeagus, outer pair shorter, upcurved; aedeagus with lateral flanges at basal half; a short, triangular apically pointed plate at base; thin lateral flaps on each side.

Female abdomen (fig. 21D) tergite IX with small lateroventral pockets at base; sternite VIII deeply divided ventromesally.

\section{Distribution}

Tasmania, NW, SW, SE and NE provinces. Tasmanian endemic. 


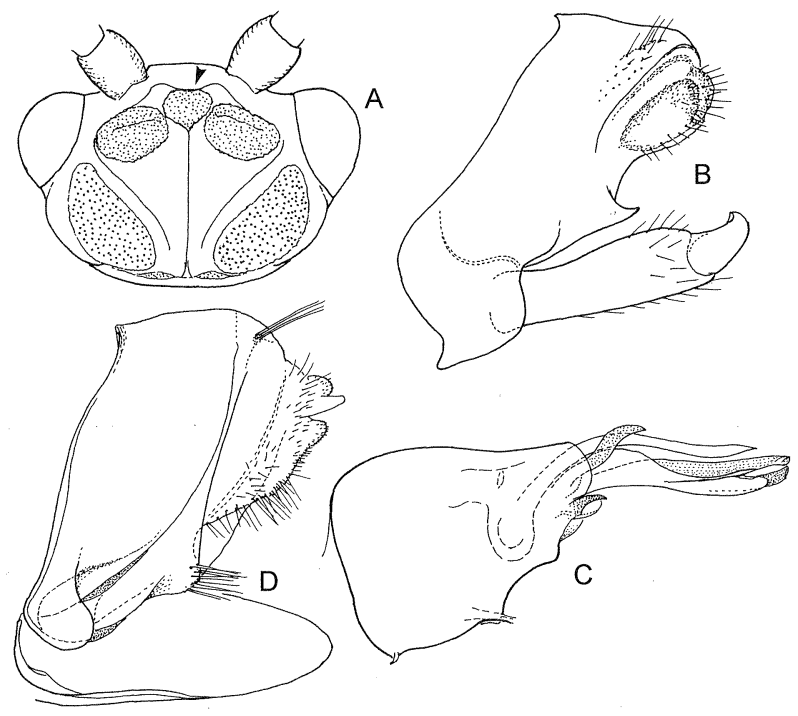

FIG. 21 - Diplectrona castanea Kimmins: (A) male head dorsal; (B) male genitalia lateral; (C) phallus lateral; (D) female genitalia lateral.

\section{Genus Diemeniluma gen.nov. Fig. 22A, B}

\section{Type species}

Diplectrona tasmanica Jacquemart, 1965, by present designation.

\section{Diagnosis}

Adult head dorsally with two pairs of large setal warts; antero-mesal wart absent. Forewing with cross-vein C-Sc present. Lateral filament on abdominal sternite $\mathrm{V}$ present in both sexes. Female abdominal sternite VIII entire, not divided ventro-mesally; segment IX without lateral scleritic cavity, sternite IX three-lobed.

\section{Description}

Wing venation (fig. 22B) regular and similar in both sexes; discoidal cell rather short and present in both wings. Forewing with forks $1-5$ present, fork 2 sessile, anal veins looped basally and only one (A-1) extends distally, reaching wing margin at arculus together with $\mathrm{Cu} 2$. Hind wing rather broad, Sc and R1 not curved, but more or less parallel to costal margin; forks 1, 2, 3 and 5 present, fork 1 with short footstalk, fork 2 sessile. Head dorsally with pair of undivided antero-lateral setal warts and larger, semi-ovoid posterolateral setal warts; antero-mesal setal wart absent. Antennae slender, about as long, or slightly shorter than forewing; segments somewhat cylindrical, each with pale encircling ring at about basal third. Lateral filaments on abdominal sternite $\mathrm{V}$ present in both sexes, slender and. extending to the distal margin of segment. In males there are two pairs of rounded membranous sacs within segments VI and VII, their diameter less than the length of the segment.

Male genitalia with segment IX annular, dorsally fused with segment $\mathrm{X}$; in lateral view a dark longitudinal inner ridge is located across segment at the base of inferior appendage. (This is not present in other Australian Diplectroninae species.) Inferior appendages slender,

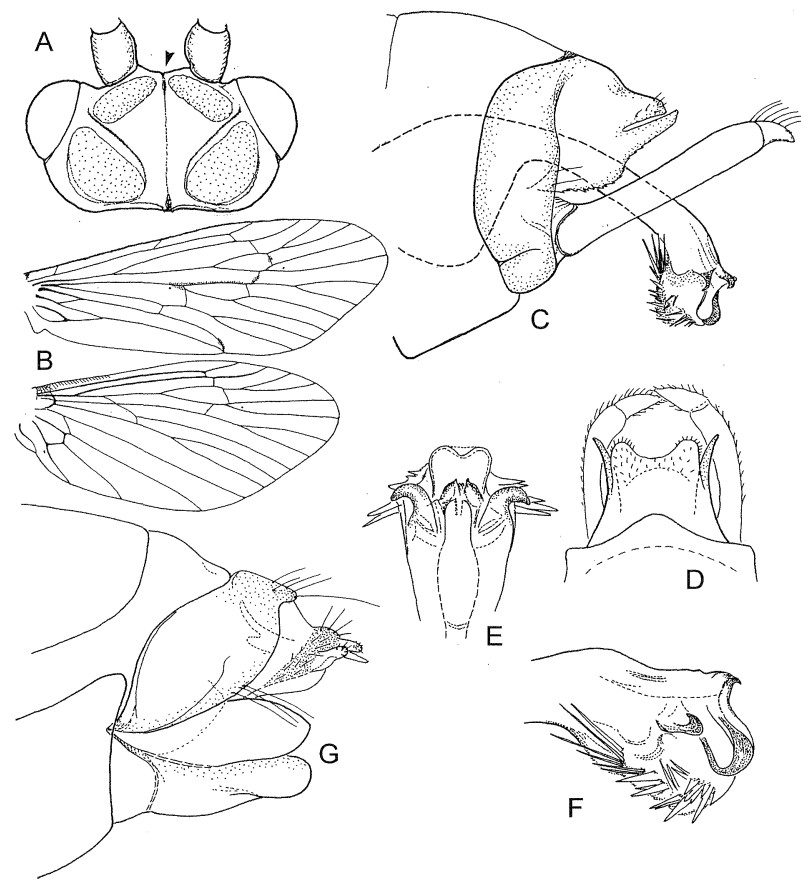

FIG. 22 - Diemeniluma tasmanica (Jacquemart): (A) male head dorsal; (B) male wing venation; $C)$ male genitalia lateral; (D) male genitalia dorsal; (E) tip of phallus ventral; $(F)$ tip of phallus lateral; $(G)$ female genitalia lateral.

harpago, small, phallus slender, broad based, apex enlarged and armed with spines.

Female differs from all other Australian Diplectroninae by having sternite VIII entire, not divided meso-ventrally; segment IX without lateral sclerotic cavity. Mid-legs not dilated.

This genus is restricted to Tasmania, where it is represented by two species. They may be separated by the shape and position of the lateral process on segment $\mathrm{X}$ and by the arrangement of spines at the apex of the phallus.

\section{Etymology}

Luma (Latin) - thorn, in reference to the spiny apex of phallus, in combination with Tasmania's original name, Van Diemen's Land.

\section{Diemeniluma tasmanica (Jacquemart) Fig. 22A-G}

Diplectrona tasmanica Jacquemart, 1965: 27; Neboiss 1977: 73, fig. 364.

\section{Holotype}

O Tasmania, Cradle Mountain, 12 Jan. 1923, A. Tonnoir (IRScNB). (Type seen.)

\section{Additional material examined}

$10^{\top} 10$ km SW of Queenstown, 11 Feb. 1971, A. Neboiss (male genitalia prep. PT-515 illustrated); 10 Gordon River, $2 \mathrm{~km}$ below Serpentine River junction, 12 Jan. 1977, A. Neboiss (female genitalia prep. PT-1067 illustrated); $10^{\star}$ Lake Tahune, Frenchmans Cap area, 28 Jan. 1988, J. Jackson. All specimens in NMV. 
Length of forewing (fig. 22B) o 6.0-6.5 mm; o $6.4 \mathrm{~mm}$; colour dark to medium brownish; median cell slightly longer than discoidal cell; fork 1 with short footstalk.

Male genitalia (fig. 22C, D) with segment IX short, lateral angles rounded, dorsally fused with segment X; apical margin of segment $\mathrm{X}$ with broad mesal recess, produced distally; lateral digital process slightly exceeding the mesal section. Inferior appendages long, harpago short, curved inward, apically pointed. Phallus slender, curved downward, terminating with apico-ventral cluster of spines, larger, curved apico-lateral spine on each side of apical sclerite.

Female (fig. 22G) tergite IX without scleritic cavity; sternite IX semi-membranous, 3-lobed.

\section{Distribution}

Tasmania, known from NW and northern SW provinces (Neboiss 1977). Tasmanian endemic.

\section{Diemeniluma serrula sp. $\mathrm{n}$. Fig. 23A-E}

\section{Type material}

Holotype o Tasmania, Hartz Mountains, Jan. 1977, J. Sedlacek. Paratypes 40 collected with holotype (male genitalia prep. PT-634 illustrated), NMV.

Length of forewing: $0^{\pi} 5.9-6.3 \mathrm{~mm}$; colour dark brownish. Male genitalia (fig. 23A-C) similar to those of $D$. tasmanica but differing in details. Segment X in dorsal view produced, almost hood-like, with small narrow cleft apically; lateral digital process situated slightly lower than in tasmanica; lower margin of segment irregularly serrate. Inferior appendages slender, harpago short, curved inward, pointed apically. Phallus slender, curved downward, apicolateral spines straight, mid-laterally three short, distally directed spines on each side.

Female unknown.

\section{Etymology}

Serra (Latin) - saw, in reference to the serrate segment X.

\section{Distribution}

Known from type locality only in the eastern part of SW province of Tasmania. Tasmanian endemic.

\section{FAMILY PLECTROTARSIDAE}

\section{Genus Plectrotarsus Kolenati}

Plectrotasrsus Kolenatis 1848: 94; Mosely \& Kimmins 1953: 21; Neboiss 1977: 75.

\section{Type species}

Plectrotarsus gravenhorsti Kolenati, 1848 by monotypy.

\section{Plectrotarsus gravenhorsti Kolenati}

Plectrotarsus gravenhorsti Kolenati 1848: 94; Neboiss 1977 : 75; 1987: 135
Holotype

Oे 'Australia occidentali' in Naturhistorisches Museum, Vienna.

\section{Additional material examined}

Tasmania, 50" $\mathrm{O}$ Hatfield River on Murchison Highway, 20 Jan. 1976, A. Wells; 1o small creek W side Great Lake, 17 Jan. 1976, A. Wells. All specimens in NMV.

\section{Distribution}

Tasmania, scattered localities NW, N, C and SE provinces. Other states: Victoria, New South Wales.

\section{Plectrotarsus tasmanicus Mosely}

Plectrotarsus tasmanicus Mosely 1936: 396; Mosely \& Kimmins 1953: 21; Neboiss 1977: 76.

Holotype

ơ Tasmania, Miena, Dec. 1930 (BMNH).

\section{Additional material examined}

Tasmania, ơ o (numerous) Lake Ayr, Cradle MountainLake St Clair National Park, 16 Jan. 1990, J. Dean and D. Cartwright; $10^{*}$ Iris River, Cradle Mountain National Park, 19 Jan. 1976, A. Wells; 1 L Lake Tahune, Frenchmans Cap area, 28 Jan 1988, J. Jackson; $20^{\top}$ Lake Whitham, Frenchmans Cap area, 27 Jan. 1988, J. Jackson; $0^{\star}$ (numerous) lake on right bank of Gordon River $8 \mathrm{~km}$ below Butler Island, 9 Jan. 1977, D. Coleman and A. Neboiss; $0^{\star}$ o (numerous) Cradle Valley, 13 Jan. 1976, A. Wells (on Leptospermum); $10^{7}$ Little Pine Lagoon, 16 Feb. 1967, E.F. Riek; O' $O$ (numerous) and larval cases, Artichoke Valley, Frenchmans Cap area, 29 Jan. 1988, J. Jackson; O’ $ᄋ$ (numerous) Swamp near Olga River, $19 \mathrm{~km}$ above Gordon River junction, 13 Jan. 1977, A. Neboiss. All specimens in NMV.

\section{Distribution}

Tasmania, C, NW and SW provinces and King Island. Tasmanian endemic.

\section{Genus Liapota Neboiss}

Liapota Neboiss 1959: 95.

Type species

Liapota lavara Neboiss, 1959 by original designation.

\section{Liapota lavara Neboiss}

Liapota lavara Neboiss 1959: 95; 1977: 76.

\section{Holotype}

o Tasmania, Cradle Mountain, (no date), J. Carter and A.M. Lea (SAM).

\section{Distribution}

Tasmania, widely distributed throughout the western provinces of the state. Tasmanian endemic. 


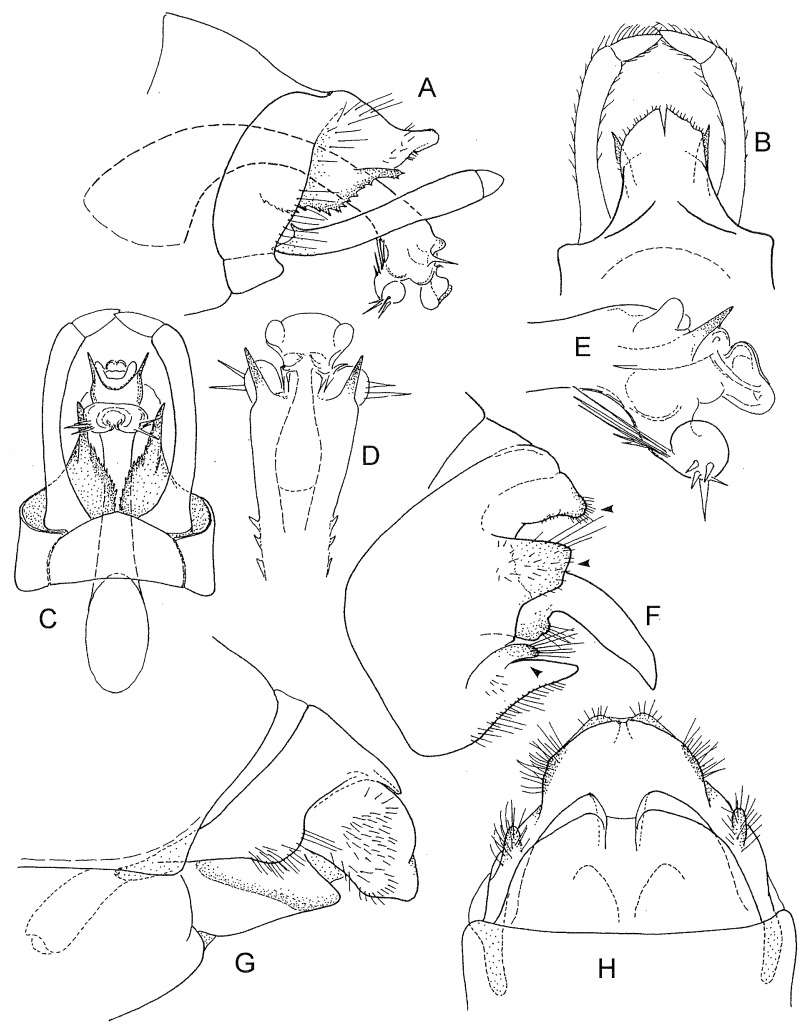

FIG. 23- $(A-E)$ Diemeniluma serrula sp. n., male genitalia: (A) lateral; (B) dorsal; $(C)$ ventral; (D) tip of phallus ventral; (E) tip of phallus lateral. (F) Archaeophylax ochreus Mosely, male genitalia lateral. $(G, H))$ Tascuna ignota Neboiss, female genitalia: $(G)$ lateral; $(H)$ ventral.

\section{Genus Nanoplectrus Neboiss}

Nanoplectrus Neboiss 1977: 77.

Type species

Nanoplectrus truchanasi Neboiss, 1977 by original designation.

\section{Nanoplectrus truchanasi Neboiss}

Nanoplectrus truchanasi Neboiss 1977: 77.

\section{Holotype}

OT Tasmania, Junction Creek, West Arthur Plains, 6 Feb. 1966 (NMV).

\section{Distribution}

Tasmania, endemic (known only from the type locality, from a single male specimen).

\section{FAMILY LIMNEPHILIDAE}

\section{Genus Archaeophylax Kimmins}

Archaeophylax Kimmins in Mosely \& Kimmins, 1953: 27; Neboiss 1977: 78.
Type species

Archaeophylax ochreus Mosely in Mosely \& Kimmins, 1953 by original designation.

\section{Archaeophylax ochreus Mosely} Fig. 23F

Archaeophylax ochreus Mosely in Mosely \& Kimmins 1953: 27; Neboiss 1977: 78.

Archaeophylax vernalis Neboiss 1977: 79. New synonym.

\section{Holotype}

ơ of Archaeophylax ochreus, Tasmania, Cuvier River, Feb. 1941 (BMNH).

ot of Archaeophylax vernalis Tasmania, Lake Pedder, 12 Mar. 1972 (NMV).

In recent years a considerable number of specimens have been accumulated in collections from numerous localities throughout the western provinces of Tasmania. Detailed examination of this material shows that male genitalic structures are variable in size and shape (as indicated by arrows on fig. $23 \mathrm{~F}$ ), the variability fully overlapping the putative distinguishing features of $A$. vernalis. Thus, the name Archaeophylax vernalis is here suppressed as a junior synonym of Archaeophylax ochreus.

Additional localities

$20^{\star} 1 \%$ Old River and Collingwood River junction, 10 Feb. 1988, A. Neboiss; $40^{\top}$ Sandfly Creek at Scotts Peak road, 9 Feb. 1988, K.Walker and J. Jackson; $10^{\pi}$ Dozer Creek 100 $m$ downstream of Gordon road, 4 Mar. 1999, J. Jackson; $30^{7}$ 1이 Lake Tahune, Frenchmans Cap area, 28 Jan. 1988, J. Jackson; $40^{*}$ Maria Creek nr Lake Pedder, 10 Feb. 1999, J. Jackson; $10^{\star}$ Scotts Peak Dam, Serpentine impoundment, 23 Mar. 1985, P. Greenslade; $10^{\star \top}$ Arve River, 3 Feb. 1983, J.C. Cardale; $10^{\top}$ Little Florentine River upstream of Gordon road, 2 Dec. 1998, J. Jackson; $10^{\pi}$ same loc., 26 Jan 1999, J. Jackson; $10^{7}$ Huon River at Tahune Bridge, 14 Jn. 1988, SC/ CF; $10^{\top}$ E-Arthur Ranges, $1 \mathrm{~km}$ W of Federation Peak, alt 1000 m, 11 Feb. 1988, P.B. McQuillan; 40 Wedge River above Gordon road, 26 Jan. 1999, in forest, J. Jackson; $30^{\star}$ Franklin River upstream of Lyell Highway, 16 Feb. 1999, J. Jackson; $30^{\star}$ Condominion Creek, Scotts Peak Dam road, 26 Jan. 1999, J. Jackson; $10^{\top}$ Dozer Creek below Gordon road, 4 Mar. 1999, J. Jackson; $10^{\star}$ Doctors Creek at Lyell Highway nr Breona, 9 Feb. 2001, R. Walker; $10^{\star}$ Pinetree Rivulet, Lake Highway nr Breona, 9 Feb. 1001, R. Walker. All specimens in NMV.

\section{Distribution}

Tasmania, widespread. Other states: Victoria, New South Wales.

\section{FAMILY OECONESIDAE}

\section{Genus Tascuna Neboiss}

Tascuna Neboiss, 1975: 82; Neboiss 1977: 83.

Type species

Tascuna ignota Neboiss, 1975 by original designation. 


\section{Tascuna ignota Neboiss Fig. 23G, H}

Tascuna ignota Neboiss, 1975: 82; Neboiss 1977: 83.

\section{Holotype}

oT Tasmania, Waldheim, Cradle Mountain National Park, 7 Feb 1971 (NMV).

\section{Additional material examined}

$10^{\pi}$ near Timber Creek close to Lake Pedder, 10 Feb. 1999 , J. Jackson; $30^{\top}$ Condominium Creek, Scotts Peak Dam road, 26 Jan. 1999, J. Jackson; $20^{\top}$ Red Tape Creek, Scotts Peak Dam road, 26 Jan. 1999, J. Jackson; $20^{*}$ Strathgordon, Jan. 1977, J. Sedlacek; 50 Old River and Collins River junction, 10 Feb. 1988, A. Neboiss; $10^{\star}$ Franklin-Gordon River junction, ANZSES expedition base camp, 20 Jan, 1983; $10^{\circ}$ Ropeway Creek, $400 \mathrm{~m}$ below Smith and Gordon River junction, 2 Feb. 1977, D. Coleman et al., 1 o Roaring CreekFranklin River junction, 8 Jan. 1977, A. Neboiss et al. (q genitalic prep. PT-1377 illustrated); $10^{\pi}$ Wedge River above Gordon road, 26 Jan. 1999, J. Jackson; $10^{\star}$ Pebbly Creek, 50 m upstream from Lake Pedder, 25 Nov. 1998, J. Jackson; $10^{\circ}$ same loc., 10 Feb. 1999, J. Jackson. All specimens NMV.

Length of forewing: $0^{7} 14.5-17 \mathrm{~mm}$; $18 \mathrm{~mm}$.

Description of female: in general appearance similar to male, but wing colouring and mottling appear to be less intense. Female abdomen bluntly rounded apically (fig. $23 \mathrm{G}, \mathrm{H}$ ); sternite VI with small gland opening laterally near anterior margin; tergite $\mathrm{X}$ produced mesally, somewhat triangular; sternite X flattened ventrally; lateral pockets are situated between apico-lateral angle of tergite IX and tergite X.

\section{Distribution}

Tasmania, restricted to NW and SW provinces. Tasmanian endemic.

\section{FAMILY CONOESUCIDAE}

The family Conoesucidae, together with Helicophidae, Calocidae and Antipodoecidae, was studied in detail by Jackson (1991) in her unpublished $\mathrm{PhD}$ thesis. The classification was analysed, immature stages described and family relationships discussed. Chromosome analyses were applied for elucidation of phylogenetic relationships. The family Conoesucidae in Tasmania is represented by five genera and 19 species. Because of lack of material Jackson (1991) excluded Costora iena Mosely and Lingora vesca Neboiss. She also suggested that $L$. vesca may simply be a malformed Lingora aurata Mosely (Jackson, pers. comm.) because further specimens collected at the type locality of vesca proved to be typical $L$. aurata.

The genus Costora in Tasmania is represented by eight species. Jackson (1991) recorded a considerable number of additional localities for almost all species. It is rather surprising, however, that Costora iena Mosely has not been recorded since its original capture in 1934. All other species are widely distributed with the exception of Costora krene Neboiss, which appears to be restricted to the SW province, between Strathgordon and Melaleuca. Only two species Costora delora Mosely and Costora ebenina Neboiss are known to occur also in SE mainland Australia; all others are endemic to Tasmania.
The validity of the Lingora-Hampa-Matasia group of genera was also questioned by Jackson (1991). The generic separations are based on male characters alone (Mosely \& Kimmins 1953), and studies of females and immature stages indicate closer relationships (Jackson 1991). Further studies are required to resolve this question.

\section{Genus Conoesucus Mosely}

Conoesucus Mosely, 1936; Neboiss 1977: 109.

\section{Type species}

Conoesucus fromus Mosely by original designation.

Key to Tasmanian species of Conoesucus males (based on the key given by Neboiss (1977), with addition of species described by Jackson (1998)):

1. Abdominal sternite VII with broad spatulate ventral process ..................................................... Abdominal sternite VII without broad spatulate process 2

Abdominal segment IX with lateral margin produced into a subtriangular lobe .................. norelus Abdominal segment IX with lateral margin curved fromus

3. Abdominal segment $\mathrm{X}$ in lateral view slightly curved upwards, margins parallel, apex broad and rounded .............................................. digitiferus Abdominal segment $\mathrm{X}$ in lateral view with margins not parallel, broadening, then tapering to apex ....... 4

4. Abdominal segment $\mathrm{X}$ turned upwards at almost a right angle, tapering to somewhat triangular apex ... 5 Abdominal segment $X$ turned only slightly upwards, apex triangular, pointed, segment IX dorsal process stout. notialis

5. Maxillary palp segment 3 completely lacking sclerotisation .............................................. nepotulus Maxillary palp segment 3 sclerotised at base ........... 6

6. Maxillary palp segment 3 about $3 X$ length of segment 2 ............................................ brontensis Maxillary palp segment 3 about equal in length to segment 2 .................................. adiastolus

\section{Conoesucus fromus Mosely Fig. 24A}

Conoesucus fromus Mosely, 1936: 409; Mosely \& Kimmins 1953: 88; Neboiss 1977: 109; Jackson 1991: 54.

\section{Holotype}

O` Tasmania, Miena, Great Lake. Dec. 1930 (BMNH).

\section{Distribution}

Tasmania, widespread, including Flinders Island; not recorded from mid-east and coastal southwest. Not recorded outside Tasmania. 


\section{Conoesucus digitiferus Jacquemart} Fig. 24B

Conoesucus digitiferus Jacquemart, 1965: 9; Neboiss 1977: 111; Jackson 1991: 53.

Holotype

O Tasmania, Cradle Mountain, 12 Jan. 1923 (IRScNB).

Distribution

Tasmania, widespread in NW and SW provinces, few records from SE province - locally common. Not recorded outside Tasmania

\section{Conoesucus norelus Mosely Fig. 24C}

Conoesucus norelus Mosely in Mosely \& Kimmins, 1953: 90; Neboiss 1977: 110; Jackson 1991: 56.

\section{Holotype}

ơ Tasmania, New Norfolk, Mar. 1938 (BMNH).

\section{Distribution}

Tasmania, widespread. Not recorded outside Tasmania.

\section{Conoesucus brontensis Neboiss} Fig. 24D

Conoesucus brontensis Neboiss, 1977: 112; Jackson 1991: 52.

\section{Holotype}

o Tasmania, $5 \mathrm{~km} \mathrm{~W}$ of Bronte, 8 Nov. 1972 (NMV).

\section{Diagnosis}

Distinguished from adiastolus by sharply upturned apex of segment $\mathrm{X}$ and maxillary palp with segment 3 long and sclerotised at base.

\section{Distribution}

Tasmania, scattered through western half of the state, but rather rare. Not recorded outside Tasmania.

\section{Conoesucus nepotulus Neboiss Fig. 24E}

Conoesucus nepotulus Neboiss, 1977: 111; Jackson 1991: 55.

\section{Holotype}

OT Tasmania, Dip River Falls, 1 Dec. 1974 (NMV).

\section{Distribution}

Tasmania - widespread through western part of the State. Not recorded outside Tasmania.

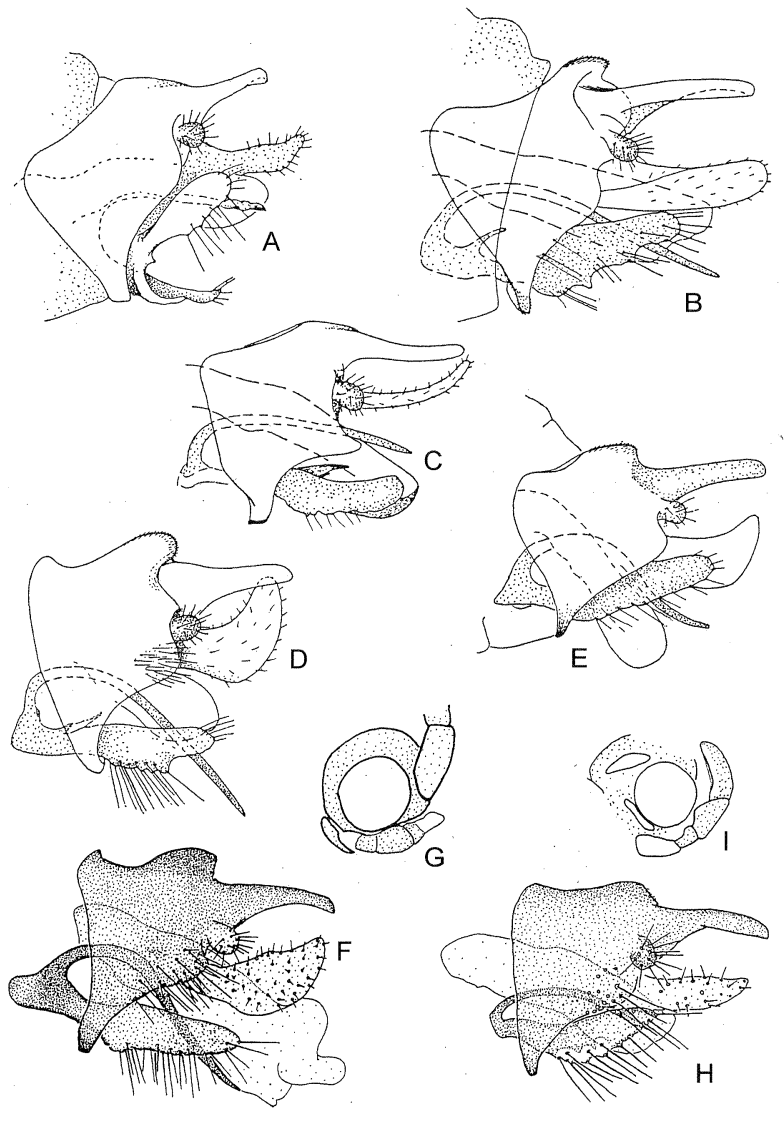

FIG. 24 - Conoesucus spp. $(A-F)$ male genitalia lateral: (A) C. fromus Mosely; (B) C. digitiferus Jacquemart; (C) C. norelus Mosely; (D) C. brontensis Neboiss; (E)C. nepotulus Neboiss; (F) C. adiastolus Jackson [from Jackson 1998]. (G) C. adiastolus Jackson [from Jackson 1998], male head lateral. (H) C. notialis Jackson [from Jackson 1998], male genitalia lateral. (I) C. notialis Jackson [from Jackson 1998], male head lateral.

\section{Conoesucus adiastolus Jackson Fig. 24F, G}

Conoesucus adiastolus Jackson, 1998: 134; Jackson 1991: 50.

\section{Holotype}

ot Tasmania, Gordon River, $2 \mathrm{~km}$ downstream of Serpentine junction, 12 Jan. 1977 (NMV).

\section{Diagnosis}

Abdominal sternite VII without broad spatulate process; segment $\mathrm{X}$ apically turned upwards; maxillary palp segment 3 about as long as segment 2, basal part sclerotised (fig. $24 \mathrm{G})$.

\section{Distribution}

Tasmania, SW province, locally abundant. Not recorded outside Tasmania. 


\section{Conoesucus notialis Jackson}

Fig. $24 \mathrm{H}, \mathrm{I}$

Conoesucus notialis Jackson, 1998: 138; Jackson 1991: 58.

Holotype

OT Tasmania, Twin Creeks, Scotts Peak Dam road, 25 Aug. 1988 (NMV).

\section{Diagnosis}

Abdominal sternite VII without broad spatulate process; dorsal processes of tergite IX stout, maxilliary palp segment 3 about as long as segments 1 and 2 together (fig. 24I).

Distribution

Tasmania, SW province. Not recorded outside Tasmania.

\section{FAMILY CALOCIDAE Genus Caloca Mosely}

Caloca Mosely in Mosely \& Kimmins, 1953: 153; Neboiss 1977: 90.

\section{Type species}

Caloca straminea Mosely, 1953 by original designation.

\section{Caloca tertia Mosely}

Caloca tertia Mosely in Mosely \& Kimmins, 1953: 156; Neboiss 1977: 90.

\section{Holotype}

ơ Tasmania, Mt Wellington, Dec. 1937 (BMNH).

\section{Distribution}

In addition to the type locality the species is now known from several additional localities thus considerably expanding the known distribution: Louisa Creek, South Coast Track; Adams River; Nelson River at Lyell Highway; Alma Tier, unnamed creek near Lake Sorell; Strathgordon and Hartz Mountains National Park.

Distribution

Tasmania, SE, SW, C and NW provinces. Tasmanian endemic.

\section{FAMILY KOKIRIIDAE}

\section{Genus Taskiria Neboiss}

Taskiria Neboiss, 1977: 80.

Type species

Taskiria austera Neboiss, 1977 by original designation.

\section{Taskiria austera Neboiss}

Taskiria austera Neboiss, 1977: 81.

\section{Holotype}

OT Tasmania, West Arthur Plains, 6 Feb. 1965 (NMV).

Additional localities

Tasmania, $10^{\top}$ South Coast, Louisa Plains, open buttongrass, 28 Dec. 1978, B.T.; $30^{\top}$ Strathgordon, Jan. 1977, J. Sedlacek; $20^{*}$ Swamp near Olga River, $19 \mathrm{~km}$ above Gordon River junction, 13 Jan. 1977, A. Neboiss; $10^{\star}$ New River Lagoon, buttongrass, 15 Feb. 1988, J. Jackson; $10^{\star}$ Franklin River, buttongrass plain, 14 Jan. 1983, ANZSES expedition; $10^{\star}$ King William Creek, Site 5A, pitfall, 21 Feb. 2001, M. Driessen; 10 King William Creek, Site 5B, sweep, 21 Feb. 2001, M. Driessen. All speciemns in NMV.

\section{Distribution}

Tasmania, NW and SW provinces, locally common. Tasmanian endemic.

\section{Taskiria mccubbini Neboiss Fig. 25A, B}

Taskiria mccubbini Neboiss, 1977: 81.

\section{Holotype}

o Tasmania, Lake Pedder (before flooding), 1 Feb. 1965 (NMV).

\section{Additional localities}

Tasmania, $20^{*}$ Lake Pedder impoundment, shore $\mathrm{N}$ of Forest Creek, 25 Nov. 1998, J. Jackson, (male gentitalia prep. PT-2141 illustrated), NMV.

\section{Distribution}

Tasmania, SW province, rare. Tasmanian endemic.

\section{Genus Taskiropsyche Neboiss}

Taskiropsyche Neboiss, 1977: 82.

Type species

Taskiropsyche lacustris Neboiss, 1977 by original designation.

\section{Taskiropsyche lacustris Neboiss Fig. 25C-E}

Taskiropsyche lacustris Neboiss, 1977: 82.

Holotype

o Tasmania, Lake Pedder (before flooding), 1 Feb. 1965 (NMV).

\section{Additional localities}

Tasmania, $10^{\star}$ stream, $300 \mathrm{~m}$ W of Teds Beach turnoff, 14 Jan. 1999, J. Jackson (male genitalia prep. PT-2198 illustrated), (NMV). 


\section{Remarks}

The specimen from Teds Beach turnoff has more strongly developed spurs (fig. 25D), and longer hairs on the legs, than specimens in the type series. The genitalia, however, conform fully with those of the type. The type series was collected at the eastern beach of Lake Pedder before flooding.

Distribution

Tasmania, SW province, rare. Tasmanian endemic.

\section{FAMILY ODONTOCERIDAE}

\section{Genus Marilia Fr. Müller}

Marilia Fr. Müller, 1878: 127.

\section{Type species}

Marilia major Fr. Müller, 1878 by subsequent designation.

The first record of Marilia from Tasmania was that of Kimmins in Mosely \& Kimmins (1953). He referred to a single male specimen from Deloraine, collected on 28 Dec. 1884 under the name Marilia bola Mosely, but noted that it is considerably larger, although the male genitalia did not differ from Marilia bola. In the absence of any further specimens the genus was neither included nor discussed by Neboiss (1977). Some years later specimens were collected at two localities leaving no doubt that the genus Marilia is indeed present in Tasmania. These specimens also proved to be rather large, comparable with that recorded by Kimmins (1953). More recently, over the period 1998-99 larvae were collected at numerous localities by members of the Department of Primary Industries, Water and Environment team. They were examined by Ros St Clair (pers. comm.) and identified as being similar to larvae of Marilia fusca Kimmins. They were collected from more than 35 localities; only one is from the west coast, from the Henty River at the Zeehan highway bridge; a few are from the central highlands and northern lowlands, but the majority are from eastern and southeastern districts - Elizabeth, Coal, Bluff, Swan, Scamander, Macquarie and other rivers. These records indicate that the Tasmanian Marilia species is associated with larger and slower flowing streams, and, according to collection notes, the larvae inhabit edge water, only seldom the riffle zone.

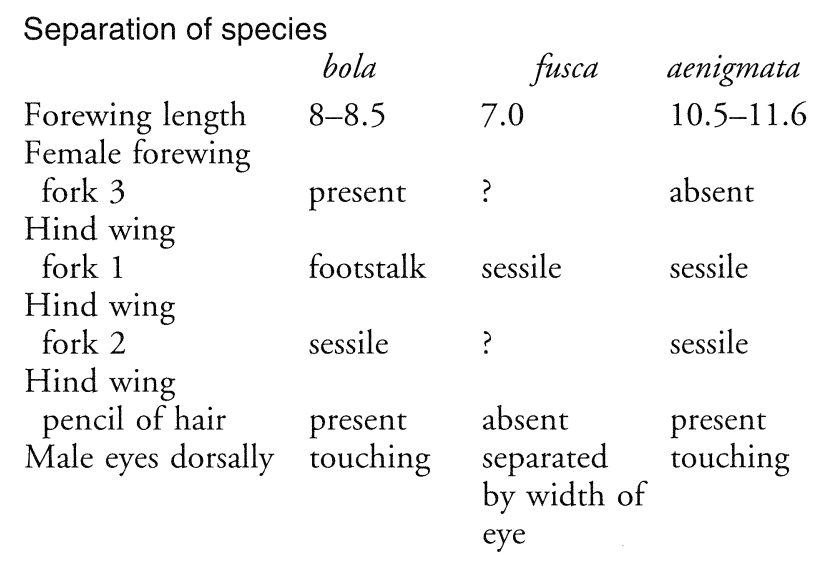

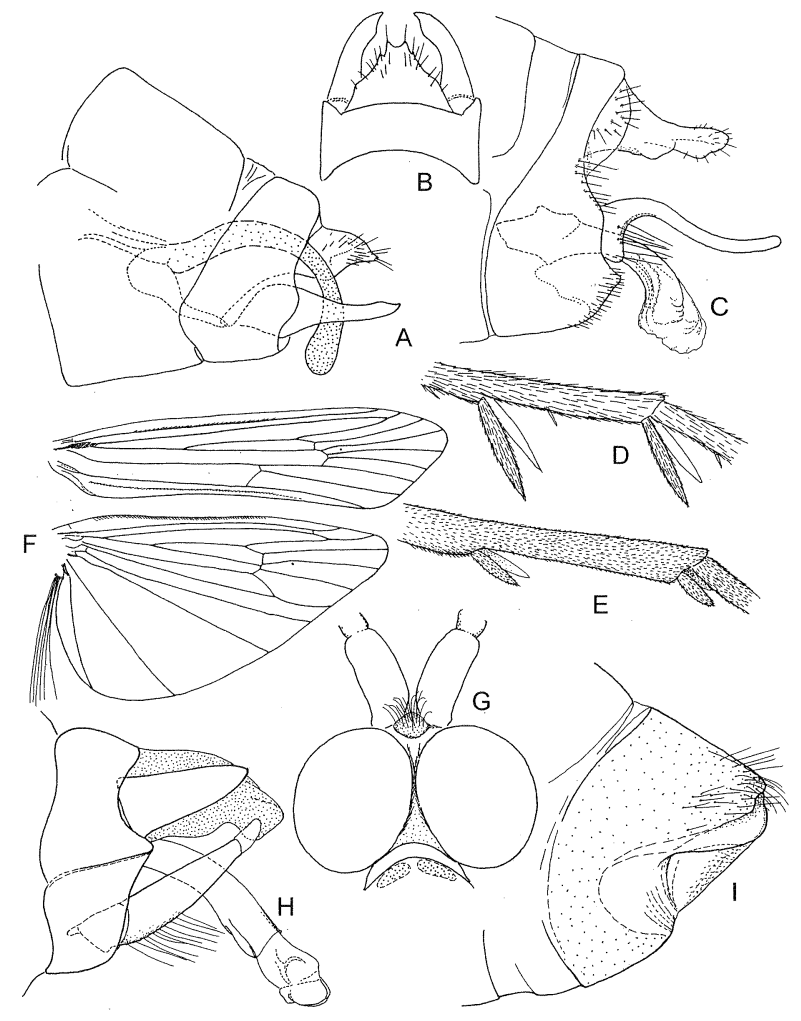

FIG. 25- $(A, B)$ Taskiria mccubbini Neboiss, male genitalia: (A) lateral; (B) dorsal. (C-E) Taskiropsyche lacustris Neboiss: (C) male genitalia lateral; (D) hind tibia - specimen from Teds Beach; (E) hind tibia - paratype from Lake Pedder east end before flooding. (F-I) Marilia aenigmata sp. n.; (F) male wing venation; $(G)$ male head dorsal; $(H)$ male genitalia lateral; (I) female genitalia lateral.

\section{Marilia aenigmata sp. $\mathrm{n}$. Fig. 25F-I}

\section{Type material}

Holotype $0^{\top}$ Tasmania, Apsley River, $5 \mathrm{~km}$ NW of Bicheno, 20 Dec. 1989, J. Jackson. Paratypes $100^{\star} 10$ o collected with holotype; 50 $594 \mathrm{~km}$ W Orford, 27 Jan. 1983, J. Cardale (wing venation and male genitalia prep. PT-1893, female genitalia prep. PT-1894 illustrated) NMV, ANIC.

\section{Description}

Forewing o 9.9-10.8 mm (fig. 25F); o 10.5-11.6 mm; dark reddish-brown with some mottling along the costal margin. Anal lobe of hind wing with pencil of long hair. Forewing forks 1 and 2 long, sessile; fork 3 absent in both sexes; fork 5 long, extending basally to about the middle of discoidal cell; hind wing with forks 1 and 2 long, sessile. In male, eyes large, globose, touching on vertex. Male genitalia (fig. $25 \mathrm{H}$ ) segment IX with short, somewhat triangular lateral projection; dorsally fused with segment $\mathrm{X}$ which terminates with a pair of apically angular lobes; superior appendages large, broad at base, narrowed apically; inferior appendages slender, 2-segmented, apical segment small; phallus slender slightly curved downward. 
Female genitalia (fig. 25I) abdominal sternite IX short; tergite IX in dorsal view somewhat triangular, produced distally; terminal segment with deep meso-ventral cleft.

Etymology

Aenigmata (L) - obscure, baffling

Distribution

Tasmania, E, C and NW provinces. Tasmanian endemic.

\section{FAMILY PHILORHEITHRIDAE}

\section{Genus Austrheithrus Mosely}

Austrheithrus Mosely in Mosely \& Kimmins, 1953: 190; Neboiss 1977: 177.

Type species

Austrheithrus dubitans Mosely, 1953 by original designation.

\section{Austrheithrus ronewa Mosely}

Austrheithrus ronewa Mosely in Mosely \& Kimmins, 1953: 192; Neboiss 1977: 117.

\section{Holotype}

$0^{7}$ New South Wales, Mt Kosciuska, alt. 2700 ft, 22 Jan. 1885 (BMNH).

\section{Distribution}

Tasmania, widespread; in addition to the northern and eastern records, this species is now recorded from the SW province: Franklin River at Roaring Creek junction and at Gordon River junction; Gordon River nr Smith River junction and above First Split; Sir John Falls; Cataract Creek; and Maxwell-Denison River junction. Other states: Victoria and New South Wales.

\section{Austrheithrus glymma Neboiss}

Austrheithrus glymma Neboiss, 1977: 118.

\section{Holotype}

OT Tasmania, St Patricks River nr Targa, 22 Feb. 1971 (NMV).

\section{Distribution}

Tasmania: to the previously recorded northern localities two are now added from the SW province - Franklin RiverRoaring Creek junction, and Gordon River at First and Second Splits. Other states: Victoria, specimens are now recorded from central and eastern Victoria, where the species appear to be widely distributed and common.

\section{Genus Tasmanthrus Mosely}

Tasmanthrus Mosely, 1936: 414; Neboiss 1977; 124.

\section{Type species}

Tasmanthrus angustipennis Mosely, 1936 by original designation.
The genus Tasmanthrus was originally described by Mosely (1936) to include a single species, Tasmanthrus angustipennis Mosely from Tasmania. The genus with its only species was discussed again in detail by Mosely $\&$ Kimmins (1953). Subsequently, Jacquemart (1965) described another species from the Cradle Mountain area. Numerous specimens from many Tasmanian localities were examined by Neboiss (1977), including the available type material of $T$. galbinomaculatus Jacquemart. He noted some small differences between the two species, but regarded them as insufficient for species separation, particularly considering the poor preservation of the galbinomaculatus type and therefore placed it as a junior synonym of angustipennis. As more extensive material became available it was recognised that some constant differences exist. Dean and Cartwright (1992) positively recognised the two species from Pelion Valley and suggested that full species status be reinstated for galbinomaculatus. St Clair (1997) separated larvae of the two species in her key. More recent field work by Jackson (1998) in the Frenchmans Cap area yielded another closely related species of Tasmanthrus which is described in this paper.

\section{Tasmanthrus angustipennis Mosely Figs 26A, 27A-F}

Tasmanthrus angustipennis Mosely, 1936: 414; Mosely \& Kimmins 1953: 183; Neboiss 1977: 124.

Holotype

ơ Tasmania, Miena, Great Lake, Dec. 1930 (BMNH).

\section{Diagnosis}

Compared with the other two species the wings are distinctly more slender (fig. 26A); in male genitalia segment X has distinctly elevated or humped meso-dorsal section; inferior appendages have the upper branch not distinctly widened at base; abdominal sternites VI and VII usually without or with very small meso-ventral protuberances.

\section{Distribution}

Tasmania widespread and common. Not recorded from other states.

\section{Tasmanthrus galbinomaculatus Jacquemart Figs 26B, 27G-I}

Tasmanthrus galbinomaculatus Jacquemart 1965: 19; Dean and Cartwright 1992: 75.

Tasmanthrus angustipennis. Neboiss 1977: 124.

Holotype

ơ Tasmania, Cradle Mountain, 12 Jan. 1923 (IRScNB).

Additional material examined

Tasmania $20^{*}$ and $20^{*}$ pupae (fully developed), unnamed Creek, Overland Track approx. $250 \mathrm{~m}$ E of Frog Flats, Cradle Mountain-Lake St Clair National Park, 18 Jan 1990, J. Dean and D. Cartwright (male genitalia prep. PT-1830 illustrated); $10^{\top} \mathrm{Mt}$ Field National Park, alt. 200 m, 4 Feb. 1980, A. Newton and M. Thayer; $10^{*}$ Little Florentine River, Southwest National Park, 22 Jan. 1976, A. Wells. 


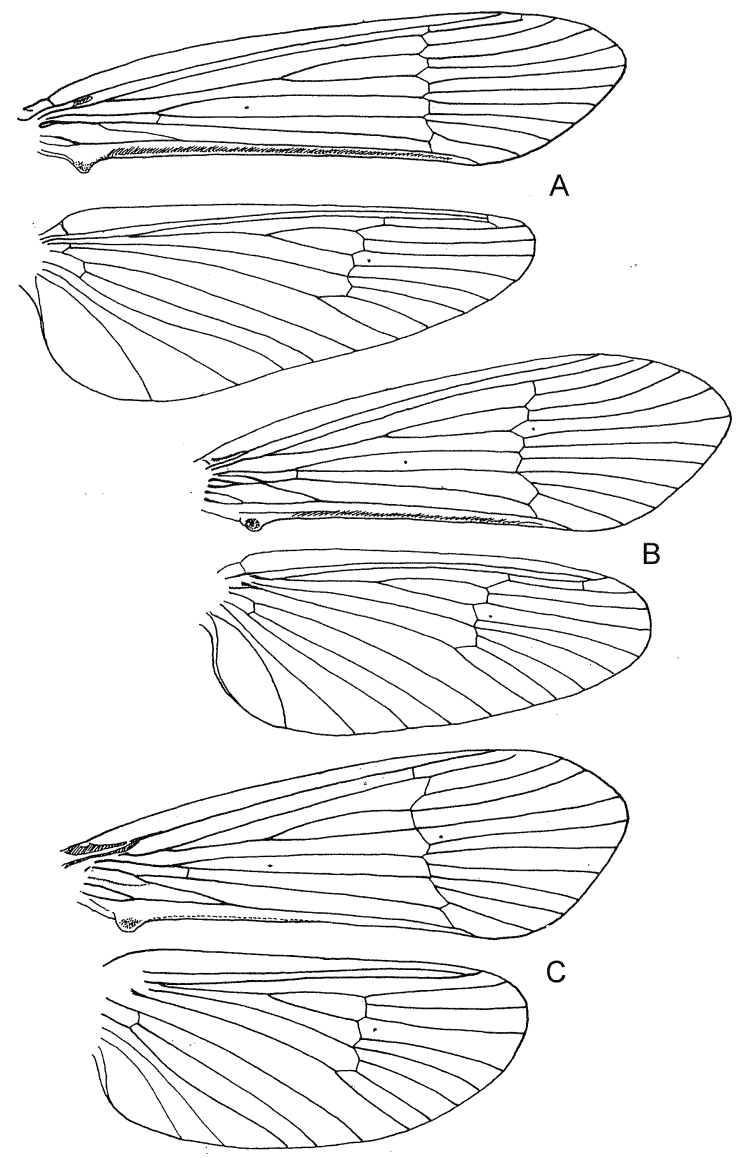

FIG. 26 - Tasmanthrus spp., male wing venation: (A) T. angustipennis Mosely; (B) T. galbinomaculatus Jacquemart; (C) T. gwendolensis sp. $n$.

\section{Diagnosis}

Wings proportionally broader than in angustipennis (fig. 26B) apex of hindwing broadly rounded. Male maxillary palpi with terminal segment elongate and tapering apically; male genitalia (fig. 27G-I) segment X meso-dorsally at most only slightly with elevated longitudinal ridge; in lateral view gradually tapering distally, only slightly curved downwards; inferior appendages upper branch distinctly widened near base; abdominal sternites VI and VII with distinct apically pointed processes.

\section{Distribution}

Tasmania. Tasmanian endemic.

\section{Tasmanthrus gwendolensis sp. $\mathrm{n}$. Figs 26C, 28A-D}

\section{Holotype}

0 Tasmania, Lake Gwendolen, Frenchmans Cap area, sweep net, 28 Jan. 1988, J. Jackson. Paratypes $30^{\star} 1$ 1 , collected with holotype (male genitalia preps PT-1697 and female PT-1881 illustrated), NMV.

\section{Description}

Adults dark blackish-brown, forewings with small pale greyish irregular spots. Length of forewing $0^{\pi} 7.4-8.6 \mathrm{~mm}$;
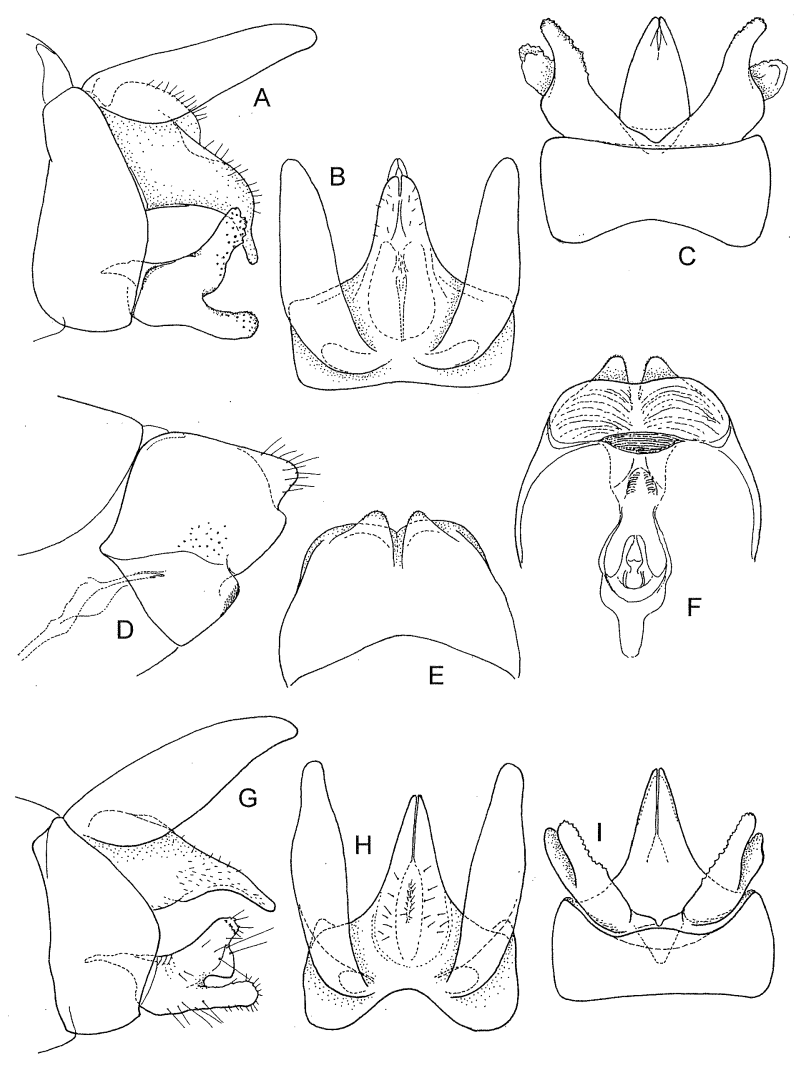

FIG. $27-(A-F)$ Tasmanthrus angustipennis Mosely. (A$C)$ male genitalia: $(A)$ lateral; $(B)$ dorsal; $(C)$ ventral. $(D-F)$ female genitalia: (D) lateral; $(E)$ dorsal; $(F)$ ventral. $(G-I)$ Tasmanthrus galbinomaculatus Jacquemart, male genitalia: (G) lateral; (H) dorsal; (I) ventral.

○ $10.8 \mathrm{~mm}$. Male genitalia (fig. 28A, B) superior appendages large, elongate ovoid, apically rounded; segment X broad at base, tapering distally, apex with $\mathrm{V}$-shaped mesal cleft, each side ending with a somewhat rounded knob; inferior appendages robust, two branched, the upper branch robust, expanded at base; abdominal sternites VI and VII with small, pointed meso-ventral processes. Phallus curved downwards.

Female abdomen (fig. 28C, D) terminates bluntly; dorsally distal end developed into two apically somewhat triangular lobes, mesally separated by comparatively broad elongate gap, which has a distinct mesal midline or ridge.

\section{Etymology}

Named after the type locality, Lake Gwendolen.

Distribution

Tasmania, known only from type locality, SW province. Tasmanian endemic.

\section{FAMILY LEPTOCERIDAE}

\section{Genus Leptorussa Mosely}

Leptorussa Mosely in Mosely \& Kimmins, 1953: 272; Neboiss 1977: 141. 


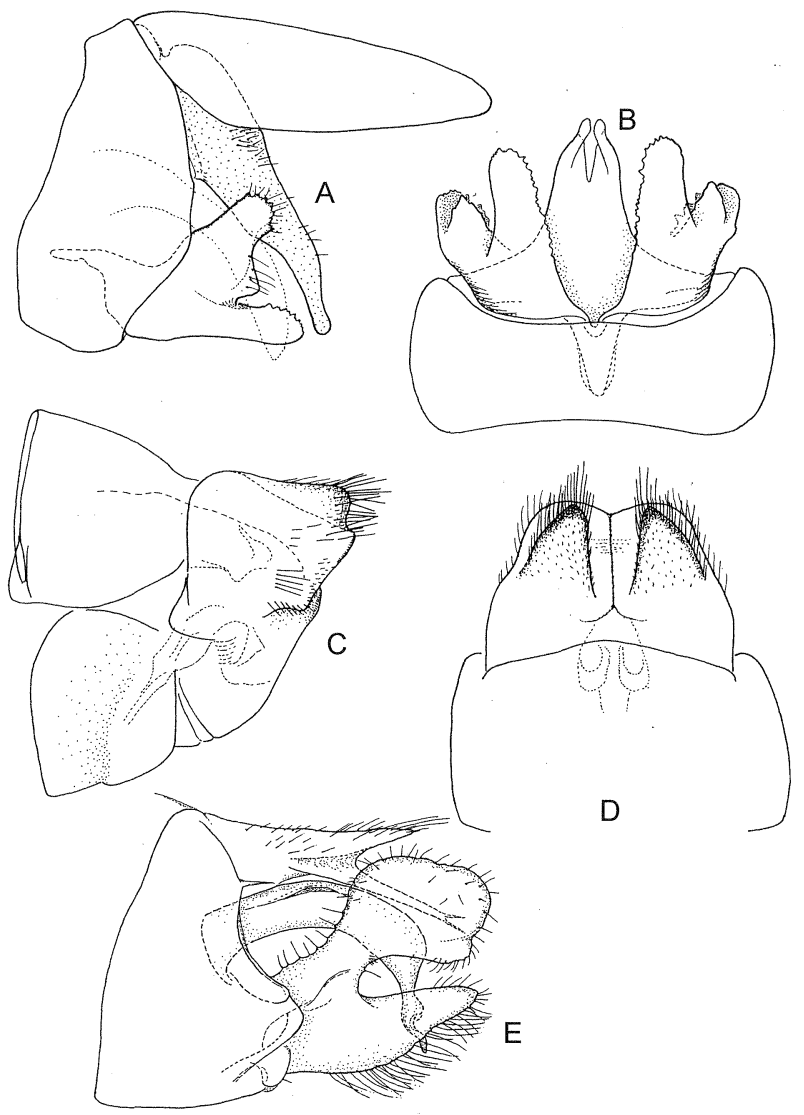

FIG. 28 - (A-D) Tasmanthrus gwendolensis sp. n. $(A, B)$ male genitalia: $(A)$ lateral; $(B)$ ventral. $(C, D)$ female genitalia: (C) lateral; (D) dorsal. (E) Leptocerus souta Mosely, male genitalia lateral.

\section{Type species}

Leptorussa russata Mosely, 1953 by original designation.

\section{Leptorussa darlingtoni (Banks)}

Leptocerus darlingtoni Banks, 1939; 487; Neboiss 1977: 141.

Leptorussa russata Mosely in Mosely \& Kimmins 1953: 274.

\section{Holotype}

O’ South Australia, Mt Lofty Ranges, 29 Nov. 1931 (ANIC).

\section{Distribution}

Tasmania widespread; in addition to previously recorded localities the following are now added: Lake Pedder, several shore locations; Maria Creek and Sandfly Creek; Franklin River at Roaring Creek junction; Wilson Creek nr Hellyer; Rubicon River nr Sassafras; Mt Field National Park. Other states: Victoria, South Australia, New South Wales.

\section{Genus Leptocerus Leach}

Leptocerus Leach, 1815: 136.

Type species

Phryganea interrupta Fabricius, 1775 by monotypy.

\section{Leptocerus souta Mosely}

(Fig. 28E)

Leptocerus souta Mosely in Mosely \& Kimmins, 1953: 278; Neboiss 1986: 255; Schmid 1987: 126.

Holotype

ơ South Australia (without locality or date) (BMNH).

The species was first recorded from Tasmania and identified positively by Neboiss in 1991, but no further adult specimens have been collected. Larvae, recorded by Ros St Clair (pers. comm.) as probably this species, were collected from the Sorell area and published by Jackson 1999.

\section{Material examined}

Tasmania, $20^{\star}$ Macquarie River, 18 Dec. 1990, S. Chilcott; larvae, Sorell Rivulet, Pawleena Reservoir nr Sorell, $42^{\circ} 43^{\prime} \mathrm{S}$, $147^{\circ} 35^{\prime} \mathrm{E}$ (no date given), NMV voucher specimen AV 5562. All material in NMV.

\section{Distribution}

Tasmania, N and SE provinces. Other states: South Australia, Victoria, New South Wales, Queensland.

\section{Genus Lectrides Mosely}

Lectrides Mosely in Mosely \& Kimmins, 1953: 270; Neboiss 1977: 135.

\section{Type species}

Lectrides varians Mosely, 1953 by original designation.

\section{Lectrides varians Mosely}

Lectrides varians Mosely in Mosely \& Kimmins, 1953: 270, Neboiss 1977: 135.

Holotype

OT Tasmania, Sheffield, 8 Jan. 1917 (BMNH).

\section{Distribution}

Tasmania, widely distributed throughout the state. Recently recorded from the following areas: Lake Tahune, Frenchmans Cap area; Mulcahy Bay, Lake Pedder several shore localities; Strathgordon; Sir John Falls; Olga River; South Esk River near Evandale; Hellyer River Gorge; Great Forester River; Apsley River near Bicheno; Russell Falls. Other states: South Australia, Victoria, New South Wales, southeast Queensland. 


\section{ACKNOWLEDGEMENTS}

The work was initiated and funded by the Tasmanian Department of Primary Industries, Water and Environment. Particular thanks are due to Michael Driessen, World Heritage Area zoologist, for his interest and support; Alice Wells, Australian Biological Resource Study (ABRS), Canberra, for encouragement and constructive criticism; Jean Jackson, Inland Fisheries Commission, Hobart, for the supply of specimens; and to John Dean and David Cartwright, Melbourne, for numerous discussions. Thanks are also due to Ken Walker, Museum Victoria, for permission to use facilities at the museum. ABRS is thanked for assistance with preparation of the manuscript.

\section{REFERENCES}

Cartwright, D.I., 1990: The Australian species of Ecnomus McLachlan (Trichoptera: Ecnomidae). Memoirs of the Museum of Victoria 51: 1-48

Cartwright, D.I. \& Dean, J.C., 1982: A key to the Victorian genera of free-living and retreat-making caddis-fly larvae (Insecta: Trichoptera). Memoirs of the Museum of Victoria 43: 1-13.

DEAN, J.C., 1997: Larvae of the Australian Hydrobiosidae (Insecta: Trichoptera). Identification Guide 11. The MurrayDarling Freshwater Research Centre, Albury. 53 pp.

DeAN, J.C., 1999: Preliminary keys for the identification of Australian Trichoptera larvae of the Family Hydropsychidae. Identification Guide 22. The MurrayDarling Freshwater Research Centre, Albury. 40 pp.

DEAN, J.C. \& BUNN, S.E., 1989: Larval descriptions of the Hydrobiosidae, Philopotamidae, Hydropsychidae and some Ecnomidae (Trichoptera) from South-western Australia, with notes on biology. Australian Journal of Marine \& Freshwater Research 40: 631-643.

Dean, J.C. \& CarTwRight, D.I., 1992: Plecoptera, Ephemeroptera and Trichoptera of the Pelion Valley, Tasmanian World Heritage Area. Occasional Papers Museum of Victoria 5: 73-79.

JACKsOn, J.E., 1991: Systematics of the Conoesucidae, Helicophidae, Calocidae and Antipodoecidae (Insecta: Trichoptera), with emphasis on immature stages. Unpubl. PhD thesis, University of Tasmania, Hobart: 119 pp.

JACKSON, J.E., 1998: Two new species of Conoesucus Mosely from Tasmania (Trichoptera: Conoesucidae). Memoirs of the Museum of Victoria 57: 133-142.

JACKSON, J.E., 1999: Trichoptera (caddisflies) of the Tasmanian Wilderness World Heritage Area. Inland Fisheries Commission. Occasional Report 99/01: 48 pp.

JACKSON, J.E., 2000: Threatened Trichoptera (caddisflies) of the Tasmanian Wilderness World Heritage Area. Papers and Proceedings of the Royal Society of Tasmania 134: 55-62.

JACQUEMART, S., 1965: Contribution à la connaisance de la faune Trichopterologique de la Tasmanie et de la NouvelleZealande. Bulletin Institut royal des Sciences naturelles de Belgique 41(35): 1-47.

Mosely, M.E., 1936: Tasmanian Trichoptera or Caddis-flies. Proceedings of the Zoological Society of London 1936: 395424

Mosely, M.E. \& Kimmins, D.E., 1953: The Trichoptera (Caddisflies) of Australia and New Zealand. British Museum (Natural History), London. 550 pp.

Neboiss, A., 1959: New caddis-fly genus from Tasmania (Trichoptera: Plectrotarsidae). Memoirs of the National Museum of Victoria 24: 91-96.

Neboiss, A., 1962: The Australian Hydrobiosidae (Trichoptera: Rhyacophilidae). Pacific Insects 4: 521-582.
Neboiss, A., 1975: The family Oeconesidae (Trichoptera) from New Zealand and Tasmania. Australian Entomology Magazine 2(4): 79-84.

Neboiss, A., 1977: A taxonomic and zoogeographic study of Tasmanian caddis-flies (Insecta: Trichoptera). Memoirs of the National Museum of Victoria 38: 1-208.

Neboiss, A., 1982: The Caddis-flies (Trichoptera) of SouthWestern Australia. Australian Journal of Zoology 30: 271 325.

Neboiss, A., 1986: Taxonomic changes in caddis-fly species from South-west Pacific-Australian region with descriptions of new species (Insecta: Trichoptera) Memoirs of the Museum of Victoria 47(2): 213-223.

Neboiss, A., 1987: Immature stages of Plectrotarsus gravenhorsti Kolenati (Trichoptera: Plectrotarsidae) and comments on its likely family relationships. Proceedings of the Royal Society of Victoria 99: 135-140.

Neboiss, A., 1993: Revised definition of the genera Nyctiophylax Brauer and Paranyctiophylax Tsuda (Trichoptera: Polycentropodidae). In Otto, C. (Ed.): Proc. 7th Int. Symposium on Trichoptera, Umea. Backhuys Publishers, Leiden: 107-111.

Nielsen, A., 1980: A comparative study of genital segments and the genital chamber in female Trichoptera. Kongelige Danske Viedenskabernes Selskab Biologiske Skrifter 23: 1; 200 pp.

SCHMID. F., 1987: Considerations diverses sur quelques genres Leptocerins (Trichoptera: Leptoceridae). Bulletin Institut Royal des Sciences Naturelles de Belgique, Entomologie 57 (suppl.) $147 \mathrm{pp}$.

Schmid. F., 1989: Les Hydrobiosides (Trichoptera, Annulipalpia). Bulletin Institut Royal des Sciences Naturelles de Belgique, Entomologie 59 (supplement) 154pp.

WARD, B.J. \& SCHEFTER, P.W., 2000: A new genus and twenty nệw species of New Caledonian Ecnomidae (Trichoptera). Records of the Canterbury Museum 14: 55-87.

Wells, A., 1978: A review of the Australian species of Hydroptila Dalman (Trichoptera: Hydroptilidae), with descriptions of new species. Australian Journal of Zoology 26: $745-$ 762.

Wells, A., 1979: A review of the Australian genera Xuthotrichia Mosely and Hellyethira Neboiss (Trichoptera: Hydroptilidae), with descriptions of new species. Australian Journal of Zoology 27: 311-329.

Wells, A., 1979: The Australian species of Orthotrichia Eaton (Trichoptera: Hydroptilidae). Australian Journal of Zoology 27: 585-622.

Wells, A., 1980: A review of the Australian genera Orphninotrichia Mosely and Maydenoptila Neboiss (Trichoptera: Hydroptilidae), with descriptions of new species. Australian Journal of Zoology 28: 627-645.

Wells, A., 1981: The genera Oxyethira Eaton, Gnathotrichia Ulmer and Stenoxyethira Kimmins (Trichoptra: Hydroptilidae) in Australia. Australian Journal of Zoology 29: 103-118.

Wells, A., 1982: Tricholeiochiton Kloet \& Hincks and new genera in the Australian Hydroptilidae (Trichoptera). Australian Journal of Zoology 30: 251-70

Wells, A., 1985: Larvae and pupae of Australian Hydroptilidae (Trichoptera), with observations on general biology and relationships. Australian Journal of Zoology Supplement 113: 69 pp.

Wells, A., 1987: Biogeography of the Oxyethira group, Tribe Hydroptilini (Hydroptilinae, Hydroptilidae, Trichoptera). Proceedings of the 5th International Symposium on Trichotera: 133-138.

Wells, A., 1998: Two new species of Hydroptilidae (Trichoptera) from Tasmania's World Heritage Area. Australian Entomologist 25(3): 81-84. 
Wells, A. 2002: Two new species of Oxyethira Eaton (Hydroptilidae: Trichoptera: Insecta) for Tasmania. Papers and Proceedings of the Royal Society of Tasmania 136: 39-41.

(accepted 29 July 2002) 